\title{
Data report: whole-rock major and trace elements and mineral compositions of the sheeted dike-gabbro transition in ODP Hole 1256 '
}

\author{
Shusaku Yamazaki, ${ }^{2}$ Natsuki Neo, ${ }^{2}$ and Sumio Miyashita ${ }^{2}$
}

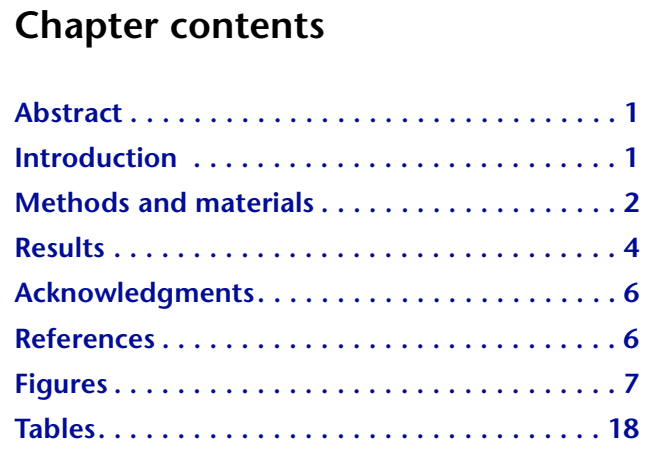

${ }^{1}$ Yamazaki, S., Neo, N., and Miyashita, S., 2009. Data report: whole-rock major and trace elements and mineral compositions of the sheeted dike-gabbro transition in ODP Hole 1256D. In Teagle, D.A.H., Alt, J.C., Umino, S., Miyashita, S., Banerjee, N.R., Wilson, D.S., and the Expedition 309/312 Scientists, Proc. IODP, 309/312: Washington, DC (Integrated Ocean Drilling Program Management International, Inc.). doi:10.2204/iodp.proc.309312.203.2009 ${ }^{2}$ Graduate School of Science and Technology, Niigata University, 8050 Ikarashi 2, Niigata 9502181, Japan. Correspondence author: shu-saku@mvd.biglobe.ne.jp

\begin{abstract}
We analyzed whole-rock major element, trace element, and mineral compositions of basalt and gabbro samples recovered from Ocean Drilling Program (ODP) Hole 1256D during Integrated Ocean Drilling Program Expedition 312. Hole 1256D, located at ODP Site 1256 in the eastern equatorial Pacific, is drilled into $\sim 15$ Ma oceanic crust at the East Pacific Rise, which was formed during a period of superfast spreading (up to $220 \mathrm{~mm} / \mathrm{y}$ full rate). The analyzed samples were collected from the upper dikes (12551348.3 meters below seafloor [mbsf]), granoblastic dikes (1348.31406.6 mbsf), Gabbro 1 (1406.6-1458.9 mbsf), the upper dike screen (1458.9-1483.1 mbsf), Gabbro 2 (1483.1-1495 mbsf), and the lower dike screen of uncertain origin (1495-1507.1 mbsf). Whole-rock compositions were measured for 19 basalts and 13 gabbros by X-ray fluorescence and inductively coupled plasmamass spectrometry. Mineral compositions were analyzed for 18 basalts and 24 gabbros by electron probe X-ray microanalysis. Results show the geochemical characteristics of each lithology and the downhole variations from the sheeted dike complex to gabbros in a superfast spreading oceanic crust. The upper dikes, granoblastic dikes, and upper dike screen have similar normal mid-ocean-ridge basalt (N-MORB)-like trace element patterns. However, the plagioclase $\mathrm{MgO}$ contents of the granoblastic dikes and upper dike screen are notably lower than those of the sheeted dikes. The lower dike screen has a unique P-, Zr-, Hf-, and light rare earth element-depleted character. On the other hand, clinopyroxene and plagioclase compositions resemble those of the granoblastic dikes and the upper dike screen. Gabbros 1 and 2 have almost the same N-MORB-normalized trace element patterns, though their $\mathrm{La} / \mathrm{Sm}$ ratios differ. Clinopyroxene $\mathrm{TiO}_{2}$ and the zoning of olivine Fo content also differ between Gabbros 1 and 2. Downhole variations show that Gabbro 1 decreases in whole-rock $\mathrm{TiO}_{2}, \mathrm{P}_{2} \mathrm{O}_{5}$, and $\mathrm{Zr}$ content and increases in pyroxene $\mathrm{Mg} \#(100 \times \mathrm{Mg} /[\mathrm{Mg}+\mathrm{Fe}])$, except for the most differentiated sample in the middle part of Gabbro 1.
\end{abstract}

\section{Introduction}

This report provides the results of geochemical analyses of basalt and gabbro shipboard samples recovered during Integrated Ocean Drilling Program (IODP) Expedition 312 from Ocean Drilling Pro- 
gram (ODP) Hole 1256D. Hole 1256D is in the oceanic crust of the Cocos plate, which was formed by superfast spreading at the East Pacific Rise $(220 \mathrm{~mm} / \mathrm{y}$ full spreading rate) at $15 \mathrm{Ma}$. Hole $1256 \mathrm{D}$ was deepened to 1507.1 meters below seafloor (mbsf) during three combined expeditions: ODP Leg 206 and IODP Expeditions 309 and 312. These expeditions succeeded in collecting gabbro samples from an upper oceanic crust sequence composed of deep-sea sediment, pillow and lava flows, and sheeted dikes.

During Expedition 312, drilling was started in the sheeted dikes at $1255 \mathrm{mbsf}$. The lowermost $60 \mathrm{~m}$ was partially or completely recrystallized. Because these sheeted dikes are characterized by distinctive granoblastic textures with secondary mineral assemblages of plagioclase, clinopyroxene, orthopyroxene, amphibole, and Fe-Ti oxide, they were classified as the "granoblastic dikes" (Wilson et al., 2006). At 1406.6 mbsf, drilling in Hole 1256D encountered a felsic plutonic rock intruded into the granoblastic dikes. This depth is regarded as the beginning of the "plutonic complex" (Wilson et al., 2006). The plutonic complex includes two gabbroic intervals $(52.3 \mathrm{~m}$ thick upper Gabbro 1 and $24 \mathrm{~m}$ thick lower Gabbro 2) separated by the $24.2 \mathrm{~m}$ thick upper dike screen (see "Igneous petrology" in the "Site 1256" chapter). The lower portion of Gabbro 2 consists of a $12.1 \mathrm{~m}$ thick gabbronorite of uncertain origin. We hereafter refer to this gabbronorite unit as the lower dike screen, which is assigned to metamorphosed basalt dike screen in the "Site 1256" chapter.

A data set of geochemical analysis of the sheeted dikes-gabbro transition in Hole 1256D gives basic information for understanding the magmatic, metamorphic, and hydrothermal systems beneath the superfast spreading ridge.

\section{Methods and materials}

\section{Sample description}

This report presents the whole-rock major and trace element compositions of 32 samples (19 basalts and 13 gabbros) and mineral compositions of 42 samples (18 basalts and 24 gabbros) obtained during Expedition 312. On the basis of microscopic observations, we selected samples with the minimum amount of secondary minerals such as chlorite, epidote, and albite so that primary igneous mineral compositions are well preserved for electron probe microanalysis. Analyzed samples are listed in Table T1.

The lithologic features of the samples are as follows. The dikes, which were recovered from Hole 1256D during Expedition 312, are metamorphosed to various grades from greenschist to pyroxene hornfels facies (see "Igneous petrology" in the "Site 1256" chapter). The development of the secondary minerals of Hole 1256D basalts and dolerites is classified into eight types in the "Site 1256" chapter and by Koepke et al. (2008). This report follows this classification, based on the primary igneous features of glass, clinopyroxene, plagioclase, and Fe-Ti oxides and secondary mineral assemblages of dusty brown material (mostly actinolite), amphibole, clinopyroxene, orthopyroxene, and Fe-Ti oxides. Type 1 is defined as completely fresh basalt with pristine glass and phenocrysts. Type 2 is characterized by the appearance of secondary minerals after glass. Type 3 is defined as slightly altered basalt where glass is altered to chlorite and oxides and $<50 \%$ of primary clinopyroxene is altered to the dusty brown fibrous masses, while plagioclase is mostly primary. Type 4 is defined as the much-altered basalt in which $>50 \%$ clinopyroxene is altered to dusty brown fibrous masses and Fe-Ti oxides and plagioclase is altered to dusty brown material. Type 5 is characterized by the first appearance of granular clinopyroxene, orthopyroxene, and green flaky hornblende as secondary minerals. This type is defined as metamorphosed basalt in which $>90 \%$ clinopyroxene is altered to actinolite and oxide and plagioclase is replaced by submicrometer-size discrete Fe-Ti oxides and actinolite. Type 6 is defined as metamorphosed basalt where primary clinopyroxene is completely altered to prismatic actinolite, green to brown flaky hornblende, and Fe-Ti oxides grains. Types 7 and 8 are mature metabasalt. Type 7 is defined by microgranular mosaic-like texture with typical granoblastic domains, flaky and poikiloblastic green to brown hornblende, and prismatic actinolite. The typical granoblastic domain consists of microgranular clinopyroxene, orthopyroxene, and Fe-Ti oxides. Type 8 metabasalt is characterized by more or less continuous microgranular granoblastic mosaics of secondary clinopyroxene, orthopyroxene, plagioclase, hornblende, and Fe-Ti oxides (for details, see table 3 of Koepke et al., 2008). These types correspond to metamorphic grade from greenschist facies (Type 3) up to pyroxene hornfels facies (Type 7 and 8). The analyzed samples include Type $3,4,6,7$, and 8 . The majority of the sheeted dikes are Type 3 and 4 basalts, except that the granoblastic dikes are Type 7 . The upper dike screen consists of Type 8 metabasalt. Under a microscope, it is not clear whether three samples (3121256D-233R-1, 4-7 cm, and 234R-1, 1-2 and 7-9 cm) from the lower dike screen are of igneous or metamorphic origin (see "Igneous petrology" in the "Site 1256" chapter). These rocks show a microgranular texture with granular clinopyroxene, orthopyroxene, plagioclase, and Fe-Ti oxide and include 0.5 to $1.5 \mathrm{~mm}$ long phenocryst-like prismatic plagioclase 
relics that include submicrometer-scale spherical FeTi oxides and transparent inclusions (Fig. F1G, F1H). Because this mineral assemblage, texture, and appearance of phenocryst relics resemble the characteristics of the Type 8 metabasalts of the upper dike screen, these samples are classified as Type 8 metabasalt.

Gabbro 1 mainly consists of disseminated oxide orthopyroxene-bearing olivine gabbro. Gabbro 1 gabbros commonly carry Fe-Ti oxide, which decreases with depth. Olivine is present in significant amounts in the lower portions of Gabbro 1, whereas orthopyroxene is not abundant and is largely associated with olivine. Some of the large orthopyroxenes include numerous bleb-like clinopyroxene intergrowths a few micrometers in size. The clinopyroxenes of Gabbro 1 are anhedral or poikilitic between subhedral plagioclase frameworks. Multiple zoning is observed in large subhedral plagioclases. Gabbro 1 clinopyroxenes are largely altered to green or greenish brown amphibole with Fe-Ti oxide. The upper part of Gabbro 1 (Units 83 and 85) has the development of a patchy texture with clinopyroxene-rich subophitic domain and plagioclase-rich coarse-grained patch or network domain (Fig. F32 in the "Site 1256" chapter). The subophitic domain is characterized by clinopyroxene oikocrysts roughly 5 to $10 \mathrm{~mm}$ across with up to a few millimeter long plagioclase chadocrysts. The coarse-grained domain is characterized by coarser grained zoned euhedral prismatic plagioclase and abundant Fe-Ti oxide (Fig. F2). The subophitic domain rarely contains orthopyroxene. Remarkable exsolution lamellae occur in some of the clinopyroxenes in the coarse-grained domain. The clinopyroxene with the lamellae occurs in the rim of the subophitic clinopyroxene at the contact between the two domains (Fig. F1A, F1B). The alteration minerals in Gabbro 1 are dominated by albite, chlorite, epidote, calcite, green amphibole, and Fe-Ti oxides.

Gabbro 2 mainly consists of orthopyroxene-bearing gabbro, characterized by variable orthopyroxene content up to $20 \%$. Fe-Ti oxides are present up to $5 \%$ and generally decrease with depth. Plagioclase is euhedral to subhedral with a simply or oscillatory zoned clouded core that includes numerous submicrometer opaque inclusions (Fig. F1C). In the metabasalts and Gabbros 1 and 2, clinopyroxene is classified into three types: igneous-type, amphiboleintergrowth (amphibole-type), and pale green colored (secondary-type). Igneous-type clinopyroxene is characterized by the existence of exsolution lamellae partly filled with opaque minerals (Fig. F1E). Amphibole-type clinopyroxene is variably replaced by greenish brown to brown amphiboles and Fe-Ti oxides a few to a few tens of micrometers in diameter (Fig. F1D). Secondary-type clinopyroxene is more transparent than the other types. This type is rarely observed in Type 7 metabasalt in Gabbro 1 and the upper and lower dike screen and rarely occurs as a cluster or vein (Fig. F1F). These secondary-type clinopyroxenes are observed as the pale green colored poikiloblasts which form veins or clusters and are sharply distinguished from the other types by their extremely low $\mathrm{TiO}_{2}$ content (see "Clinopyroxene"). This pale green clinopyroxene with extremely low $\mathrm{TiO}_{2}$ has been reported as secondary mineral in the granoblastic dikes because of retrograde metamorphism (Koepke et al., 2008). Gabbro 2 orthopyroxenes are anhedral and enclose or partially enclose euhedral plagioclases. Some large orthopyroxenes include clinopyroxene bleb-like intergrowths. Olivine rarely presents in Gabbro 2 and most olivine is pseudomorphed with iddingsite and iron oxide or talc, green to brown amphibole, and opaque minerals. Olivine commonly occurs with orthopyroxene and opaque minerals. Some olivines are poikilitically enclosed by large anhedral orthopyroxenes. Gabbro 2 includes some metabasalt or rounded metabasalt enclaves up to $1 \mathrm{~cm}$ in diameter (e.g., Sample 312-1256D-230R-1, 81-84 cm; 1483.81 mbsf). The texture of the enclaves corresponds to Type 7 or 8 metabasalt, which is similar to the lower dike screen. Gabbro 2 alteration minerals are dominated by greenish brown to brown amphibole and Fe-Ti oxide.

\section{Methods}

\section{Sample preparation}

Samples were prepared for X-ray fluorescence (XRF) and inductively coupled plasma-mass spectrometry (ICP-MS) as follows. To minimize the effect of alteration, visibly weathered parts and hydration veins were removed. Processed chips were washed for 15 min in an ultrasonic bath filled with ion-exchanged water and then dried for $>24 \mathrm{~h}$ in an oven at $120^{\circ} \mathrm{C}$. Dried samples were crushed using a tungsten carbide mortar followed by milling in a tungsten carbide ball mill. W, Co, Ta, and $\mathrm{Nb}$ were excluded from trace element analysis because of possible contamination from the tungsten carbide.

\section{Major elements}

Major elements $\left(\mathrm{SiO}_{2}, \mathrm{Al}_{2} \mathrm{O}_{3}, \mathrm{TiO}_{2}, \mathrm{FeO}\right.$ [total $\mathrm{Fe}$ as $\mathrm{FeO}$ ], $\mathrm{MnO}, \mathrm{CaO}, \mathrm{MgO}, \mathrm{Na}_{2} \mathrm{O}, \mathrm{K}_{2} \mathrm{O}$, and $\mathrm{P}_{2} \mathrm{O}_{5}$ ) were analyzed with a Rigaku RIX-3000 X-ray fluorescence spectrometer at the Faculty of Science, Niigata University (Japan). Powdered samples were dried at $120^{\circ} \mathrm{C}$ for $1 \mathrm{~h}$ to remove $\mathrm{H}_{2} \mathrm{O}$ and heated for a further $12 \mathrm{~h}$ at $900^{\circ} \mathrm{C}$ in a muffle furnace to obtain losson-ignition (LOI) values. A heated powder sample $(0.5 \mathrm{~g})$ was mixed with $5 \mathrm{~g} \mathrm{Li}_{2} \mathrm{~B}_{4} \mathrm{O}_{7}$ and fused in a platinum crucible at $1200^{\circ} \mathrm{C}$ to form a glass bead. 
Calibration curves were made using the Geological Survey of Japan (GSJ) igneous rock series standard samples. To include a wide range of compositions, some standard samples were synthesized by adding pure chemical agents to GSJ standard samples (for example, pure $\mathrm{TiO}_{2}$ was added to the JB-1b standard). The precision of analysis was verified by multiple measurements of the JB-2 standard (Table T2). These measured values are in good agreement with the reference values (Imai et al., 1995; Eggins et al., 1997).

\section{Trace elements}

Trace elements were analyzed using the ICP-MS Agilent 7500a at the Graduate school of Science and Technology, Niigata University. The elements measured were Sc, V, Ga, Rb, Sr, Y, Zr, Cs, Ba, La, Ce, Pr, $\mathrm{Nd}, \mathrm{Sm}, \mathrm{Eu}, \mathrm{Gd}, \mathrm{Tb}, \mathrm{Dy}, \mathrm{Ho}, \mathrm{Er}, \mathrm{Yb}, \mathrm{Lu}, \mathrm{Hf}, \mathrm{Th}$, and U. An alkali fusion method was used, following Roser et al. (2000), to avoid residue of acid-resistant minerals such as zircon. However, nitric acid was used instead of the perchloric acid used in the original method. The US Geological Survey (USGS) reference material BHVO-2 was used as a standard for the correction. The precision of the analysis was verified by multiple measurements of the USGS reference material W-2. Results are shown in Table T2.

\section{Mineral composition}

Main mineral phases were analyzed by the JXA8600SX EPMA at the Graduate School of Science and Technology, Niigata University. Plagioclase, clinopyroxene, and orthopyroxene were analyzed for $\mathrm{SiO}_{2}$, $\mathrm{TiO}_{2}, \mathrm{Al}_{2} \mathrm{O}_{3}, \mathrm{Cr}_{2} \mathrm{O}_{3}, \mathrm{FeO}, \mathrm{MnO}, \mathrm{MgO}, \mathrm{CaO}, \mathrm{Na}_{2} \mathrm{O}$, $\mathrm{K}_{2} \mathrm{O}, \mathrm{BaO}$, and $\mathrm{NiO}$ under the following analytical conditions: acceleration voltage $=15 \mathrm{kV}$, probe current $=13 \mathrm{nA}$, and beam diameter $=1 \mu \mathrm{m}$. Olivine was analyzed for $\mathrm{SiO}_{2}, \mathrm{TiO}_{2}, \mathrm{Al}_{2} \mathrm{O}_{3}, \mathrm{Cr}_{2} \mathrm{O}_{3}, \mathrm{FeO}, \mathrm{MnO}$, $\mathrm{MgO}, \mathrm{CaO}$, and $\mathrm{NiO}$ under $25 \mathrm{kV}$ and $20 \mathrm{nA}$ with a beam diameter of $\sim 3 \mu \mathrm{m}$. Both natural and synthetic oxides and silicates were used as standards. Correction was made according to the $\mathrm{ZAF}(\mathrm{Z}=$ atomic number, $\mathrm{A}=$ absorption, and $\mathrm{F}=$ fluorescence) method. Core and rim compositions were obtained from each mineral phase. Analyses of small grains and anhedral minerals were done irrespective of the core and rim. Detection limits are listed in Table T3.

\section{Results}

\section{Major elements}

Type 3 to 8 basalts and metabasalts show similar variations in the major elements, such as $\mathrm{Mg \#}$ versus
$\mathrm{SiO}_{2}, \mathrm{TiO}_{2}$, and $\mathrm{P}_{2} \mathrm{O}_{5}$ (Fig. F3). Higher grade Type 7 and 8 metabasalts have slightly lower $\mathrm{K}_{2} \mathrm{O}$ and $\mathrm{Mg \#}$ than lower grade Type 3 and 4 basalts. Type 3 and 4 samples (312-1256D-176R-1, 21-24 cm, at 1276.29 mbsf and 186R-1, 33-36 cm, at $1319.82 \mathrm{mbsf}$ ) have slightly higher LOI values (2.36 and $1.18 \mathrm{wt} \%$, respectively) and notably high $\mathrm{K}_{2} \mathrm{O}$ content $(0.16$ and $0.19 \mathrm{wt} \%$, respectively) (Fig. F3B). This indicates that some elements such as $\mathrm{K}_{2} \mathrm{O}$ and $\mathrm{Na}_{2} \mathrm{O}$ had been affected by alteration. It is noted that $\mathrm{K}_{2} \mathrm{O}$ and LOI are well correlated for those with LOI $<0.8 \mathrm{wt} \%$, whereas $\mathrm{K}_{2} \mathrm{O}$ is scattered at higher LOIs. The plots characterized by very low LOI and $\mathrm{K}_{2} \mathrm{O}$ are exclusively highly recrystallized dikes such as Type 7 and 8 metabasalts. The high-LOI $(>0.8 \mathrm{wt} \%)$ samples had no clear correlation between $\mathrm{K}_{2} \mathrm{O}, \mathrm{Na}_{2} \mathrm{O}$, and LOI. Irrespective to rock types, a strong negative correlation is apparent between $\mathrm{Mg \#}$ and $\mathrm{TiO}_{2}$ (Fig. F3C). Gabbro 1 shows wider spectra of $\mathrm{SiO}_{2}$ and $\mathrm{Mg} \#\left(\mathrm{SiO}_{2}=48.4-51.4\right.$ $\mathrm{wt} \%, \mathrm{Mg \#}=56-72)$ than Gabbro $2\left(\mathrm{SiO}_{2}=49.9-51\right.$ $\mathrm{wt} \%, \mathrm{Mg \#}=61-63)$. The highest $\mathrm{SiO}_{2}$ value (51.4 wt\%) occurs at 1440.21 mbsf in the lower middle of the Gabbro 1 subunit. This sample is a slightly altered orthopyroxene-bearing gabbro (Sample 3121256D-221R-1, 61-64 cm; $1440.21 \mathrm{mbsf})$ with notably high $\mathrm{P}_{2} \mathrm{O}_{5}(0.17 \mathrm{wt} \%)$ and $\mathrm{TiO}_{2}(1.56 \mathrm{wt} \%)$ contents. Type 8 metabasalts from the lower dike screen in the lowermost part of Hole 1256D show very low $\mathrm{P}_{2} \mathrm{O}_{5}$ contents that plot away from the main trend of $\mathrm{Mg} \#$ versus $\mathrm{P}_{2} \mathrm{O}_{5}$ variation (Fig. F3D), although they plot on the main trend in the $\mathrm{TiO}_{2}-\mathrm{Mg} \#$ diagram. Downhole variations of major and trace elements are plotted in Figure F4. The $\mathrm{SiO}_{2}, \mathrm{Mg} \#, \mathrm{TiO}_{2}$, and $\mathrm{P}_{2} \mathrm{O}_{5}$ contents of the dike screen Type 8 metabasalts plot within the range of other metabasalts. Gabbro 1 shows a decreasing trend of $\mathrm{TiO}_{2}$ and $\mathrm{P}_{2} \mathrm{O}_{5}$ downhole, except for the sample with the highest $\mathrm{P}_{2} \mathrm{O}_{5}$ content.

\section{Trace elements}

Both $\mathrm{Zr}$ and $\mathrm{Zr} / \mathrm{Y}$ show similar downhole variations to those of $\mathrm{P}_{2} \mathrm{O}_{5}$ (Fig. F4E, F4F). Type 3 to 7 metabasalts show N-MORB-like patterns for rare earth elements (REE), Sr, P, Zr, Hf, and Y (Fig. F5). Concentrations of these elements are higher in Type 6 and 7 basalts than in Type 3 and 4 basalts (Fig. F5A). U, Th, and $\mathrm{Ba}$ values of Type 3 to 7 basalts and metabasalts are slightly higher than the N-MORB values $(\mathrm{N}$ MORB-normalized $\mathrm{U}=0.8-2.08$, Th $=1.09-2.54$, and $\mathrm{Ba}=0.99-5.67)$. The two low-grade metabasalts (Samples 312-1256D-176R-1, 21-24 cm, at 1276.29 mbsf and 186R-1, 33-36 cm, at $1319.82 \mathrm{mbsf}$ ) with high LOI contents are enriched in $\mathrm{K}, \mathrm{Ba}$, and $\mathrm{Rb}$. The enrichment of these elements may be ascribed to al- 
teration. The patterns of upper dike screen Type 8 metabasalts are in accordance with the patterns of Type 3 to 7 basalt and metabasalts. On the other hand, lower dike screen metabasalts have very low concentrations of REE, Zr, Hf, P, U, and Th (Fig. F5B). The downhole variation of the N-MORB-normalized $\mathrm{La} / \mathrm{Sm}$ ratio $\left(\mathrm{La}_{[\mathrm{N}]} / \mathrm{Sm}_{[\mathrm{N}]}\right)$ shows that the lower dike screen has notably low $\mathrm{La}_{[\mathrm{N}]} / \mathrm{Sm}_{[\mathrm{N}]}$ ratios $(0.51-0.57)$ (Fig. F4G). Gabbros 1 and 2 are characterized by Zr, $\mathrm{Hf}$, and $\mathrm{P}$ depletion, except for the high- $\mathrm{P}_{2} \mathrm{O}_{5}$ sample (312-1256D-221R-1, 61-64 cm; $1440.21 \mathrm{mbsf})$ with a Zr-, Hf-, U-, and Th-enriched pattern (Fig. F5C). Gabbro 1 has higher $\mathrm{La}_{[\mathrm{N}]} / \mathrm{Sm}_{[\mathrm{N}]}$ ratios $(0.97-1.42)$ than Gabbro 2 (0.76-0.90). The Gabbro $1 \mathrm{La}_{[\mathrm{N}]} / \mathrm{Sm}_{[\mathrm{N}]}$ ratio is inversely correlated with $\mathrm{REE}, \mathrm{Zr}, \mathrm{Hf}$, and $\mathrm{P}$ concentrations (Fig. F6).

\section{Mineral compositions}

\section{Clinopyroxene}

Clinopyroxene $\mathrm{Mg} \#$ ranges from 64.1 to 86.6. The variation of clinopyroxene $\mathrm{Mg} \#$ versus $\mathrm{TiO}_{2}$ shows two domains of high- and low- $\mathrm{TiO}_{2}$ contents (Fig. F7). Phenocryst, microlite, and microgranular clinopyroxene of basalts and metabasalts show a high- $\mathrm{TiO}_{2}$ trend, with $\mathrm{TiO}_{2}$ increasing with decreasing Mg\# (Fig. F7A). Amphibole intergrowth-type and secondary-type clinopyroxene of the metabasalts are plotted in the low $-\mathrm{TiO}_{2}(<0.3 \mathrm{wt} \%)$ field (Fig. F7A, F7B). Secondary-type clinopyroxenes are found in Type 7 metabasalt, the bottom of Gabbro 1, the upper dike screen, the top of Gabbro 2, and the top of the lower dike screen (Fig. F8A). Igneous-type clinopyroxene of Gabbro 1 shows the high- $\mathrm{TiO}_{2}$ trend (Fig. F7B). Gabbro 2 igneous-type clinopyroxene also plots on the high- $\mathrm{TiO}_{2}$ trend, except some clinopyroxenes plot in the low- $\mathrm{TiO}_{2}$ field of amphibole intergrowth-type plots (Fig. F7B). The compositional range of the igneous-type clinopyroxenes in Gabbros 1 and 2 approximately equals the metabasalt phenocrysts and microlites. Gabbro 2 amphibole-type clinopyroxene has a lower $\mathrm{TiO}_{2}$ than the igneous-type clinopyroxene of Gabbros 1 and 2 . Some amphibole-type clinopyroxenes overlap the secondary-type clinopyroxene. The low- $\mathrm{TiO}_{2}$ amphibole-type clinopyroxene does not preserve the primary compositions but is of metamorphic origin. Subophitic and coarse-grained network domains in Gabbro 1 clinopyroxenes have different composition. Clinopyroxene oikocryst of subophitic domains have constant Mg\# (80.7-82.4). In contrast, coarse-grained network domains show more evolved and variable $\mathrm{Mg \#} \mathrm{(66.3-76.3)} \mathrm{than} \mathrm{the} \mathrm{subophitic}$ domains. Gabbro 1 igneous-type clinopyroxene has negative correlation between $\mathrm{TiO}_{2}$ and $\mathrm{Mg} \#$ and the lowest $\mathrm{Mg} \#$ and the highest $\mathrm{TiO}_{2}$ occurs in a coarsegrained network domain at the top of the Gabbro 1 subunit (Figs. F7B, F8A, F8B). Gabbro 2 clinopyroxene is slightly evolved toward the top of the Gabbro 2 subunit (Fig. F8A).

\section{Orthopyroxene}

Orthopyroxenes show a broad positive correlation

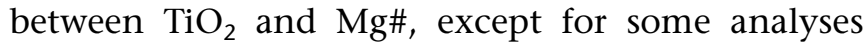
with very low $\mathrm{TiO}_{2}$ (Fig. F9A). A disseminated oxide olivine gabbro (Sample 312-1256D-215R-2, 56-59 $\mathrm{cm} ; 1417.69 \mathrm{mbsf}$ ) of Gabbro 1 includes these exceptionally low $\mathrm{TiO}_{2}$ orthopyroxenes, which occur as a cluster within an olivine pseudomorph. Gabbro 1 orthopyroxenes have a wider range of $\mathrm{Mg \#}$ (56.2-75) than those from Gabbro 2 (62.2-73.2). Gabbro 1 orthopyroxene $\mathrm{TiO}_{2}$ decreases uphole through Gabbro 1 with decreasing Mg\# (Fig. F8C). This is opposite behavior from the $\mathrm{TiO}_{2}$ versus $\mathrm{Mg} \#$ plots of the clinopyroxene (Figs. F8C, F8D, F9A). These results strongly suggest that the orthopyroxene of Gabbro 1 is of metamorphic origin.

The lowest and highest Mg\# orthopyroxenes occur at the top and bottom of Gabbro 2. In the lower part of Gabbro 2, $\mathrm{TiO}_{2}$ content increases uphole with decreasing $\mathrm{Mg \#}$. In contrast, $\mathrm{TiO}_{2}$ content in the upper part decreases uphole with decreasing Mg\# (Figs. F8C, F8D). This suggests the possibility that the orthopyroxene in the lower part of Gabbro 2 is also of metamorphic origin.

Orthopyroxenes of Type 7 and 8 metabasalts have a similar composition to Gabbros 1 and 2 with respect to $\mathrm{Mg \#}$ and $\mathrm{TiO}_{2}$. Type 8 metabasalts of the lower dike screen have a slightly higher Mg\# than Type 7 metabasalts (Fig. F9A).

\section{Olivine}

Olivine decreases in forsterite (Fo) contents with decreasing NiO. Gabbro 2 olivines have slightly lower $\mathrm{NiO}_{2}$ than those of Gabbro 1 at a given Fo. A sample from the upper part of Gabbro 1 (Sample 312-1256D-215R-1, 20-23 cm; 1415.92 mbsf) has olivine with a much higher Fo content (Fo = 76.680.2) than the other Gabbro 1 (Fo = 64.8-70.4) (Fig. F9B). Gabbro 1 olivines usually show normal zoning. In contrast, Gabbro 2 olivines are reversely zoned with Fo contents ranging from 67.3 to 74.5 (Fig. F10).

\section{Plagioclase}

Plagioclase ranges in An content from 11 to 83, and most compositions fall within $\mathrm{An}_{40-80}$. Very low An content $\left(<\mathrm{An}_{40}\right)$ is observed in some plagioclase rims 
of the upper part of Gabbro 1. These low-An plagioclases $\left(<\mathrm{An}_{40}\right)$ are not primary magmatic products and may be ascribed to secondary albitization. Plagioclase forms two groups in the $\mathrm{An}-\mathrm{MgO}$ variation diagram: high- and low-MgO groups. In the high-MgO group, $\mathrm{MgO}$ content increases with increasing $\mathrm{An} \%$, whereas in the low-MgO group, it does not vary with increasing An\% (Fig. F9C, F9D). Plagioclases of the upper and lower dike screen have very low $\mathrm{MgO}$. Most of the plagioclase does not exceed the detection limit of $\mathrm{MgO}(<0.02 \mathrm{wt} \%)$. The laths and phenocrysts of Type 3 basalts belong to the high-MgO group. The MgO content stays almost constant irrespective of An content, except the laths of one low-An sample (312-1256D-176R-1, 21-24 $\mathrm{cm} ; 1276.29 \mathrm{mbsf}$ ) (Fig. F9C). The plagioclases of Type 6,7 , and 8 metabasalts belong to the low-MgO group, where the $\mathrm{MgO}$ content does not exceed 0.1 wt $\%$ over the entire range of An. Gabbro 1 and 2 plagioclases have uniformly low $\mathrm{MgO}$ contents over a wide range in An. These plagioclases generally show normal zoning (Fig. F8E).

\section{Acknowledgments}

The authors are deeply grateful to Professor Susumu Umino of Kanazawa University and Dr. Jürgen Koepke of Leibniz Universitaet Hannover, whose enormous support and insightful comments were of inestimable value for our study. Samples were provided by the Integrated Ocean Drilling Program (IODP). Shusaku Yamazaki, Natsuki Neo, and Sumio Miyashita were supported by the Center of Deep Earth Exploration (CDEX) for travel fares. S. Yamazaki was supported by the Fellowships of the Japan Society for the Promotion of Science for Young Scientists. The authors are grateful to the Expedition 312 shipboard party and IODP staff.

\section{References}

Eggins, S.M., Woodhead, J.D., Kinsley, L.P.J., Mortimer, G.E., Sylvester, P., McCulloch, M.T., Hergt, J.M., and Handler, M.R., 1997. A simple method for the precise determination of $\geq 40$ trace elements in geological samples by ICPMS using enriched isotope internal standardisation. Chem. Geol., 134(4):311-326. doi:10.1016/ S0009-2541(96)00100-3

Imai, N., Terashima, S., Itoh, S., and Ando, A., 1995. 1994 compilation of analytical data for minor and trace elements in seventeen GSJ geochemical reference samples, "igneous rock series." Geostand. Geoanal. Res., 19(2):135-213. doi:10.1111/j.1751-

908X.1995.tb00158.x

Koepke, J., Christie, D.M., Dziony, W., Holtz, F., Lattard, D., Maclennan, J., Park, S., Scheibner, B., Yamasaki, T., and Yamazaki, S., 2008. Petrography of the dike-gabbro transition at IODP Site 1256 (equatorial Pacific): the evolution of the granoblastic dikes. Geochem. Geophys. Geosyst., 9(7):Q07O09. doi:10.1029/2008GC001939

Roser, B., Kimura, J., and Hisatomi, K., 2000. Whole-rock elemental abundances in sandstones and mudrocks from the Tanabe Group, Kii Peninsula, Japan. Shimane Diagaku Chikyu Shigen Kankyogaku Kenkyu Hokoku, 19:101-112.

Sun, S.-S., and McDonough, W.F., 1989. Chemical and isotopic systematics of oceanic basalts: implications for mantle composition and processes. In Saunders, A.D., and Norry, M.J. (Eds.), Magmatism in the Ocean Basins. Geol. Soc. Spec. Publ., 42(1):313-345. doi:10.1144/ GSL.SP.1989.042.01.19

Wilson, D.S., Teagle, D.A.H., Alt, J.C., Banerjee, N.R., Umino, S., Miyashita, S., Acton, G.D., Anma, R., Barr, S.R., Belghoul, A., Carlut, J., Christie, D.M., Coggon, R.M., Cooper, K.M., Cordier, C., Crispini, L., Durand, S.R., Einaudi, F., Galli, L., Gao, Y., Geldmacher, J., Gilbert, L.A., Hayman, N.W., Herrero-Bervera, E., Hirano, N., Holter, S., Ingle, S., Jiang, S., Kalberkamp, U. Kerneklian, M., Koepke, J., Laverne, C., Vasquez, H.L.L., Maclennan, J., Morgan, S., Neo, N., Nichols, H.J., Park, S.-H., Reichow, M.K., Sakuyama, T., Sano, T., Sandwell, R., Scheibner, B., Smith-Duque, C.E., Swift, S.A., Tartarotti, P., Tikku, A.A., Tominaga, M., Veloso, E.A., Yamasaki, T., Yamazaki, S., and Ziegler, C., 2006. Drilling to gabbro in intact ocean crust. Science, 312(5776):1016-1020. doi:10.1126/science.1126090

Initial receipt: 11 September 2008

Acceptance: 2 April 2009

Publication: 21 July 2009

MS 309312-203 
Figure F1. Microphotographs of typical rock textures of Gabbros 1 and 2 and lower dike screen. A. Boundary between subophitic and coarse-grained network domains in oxide-bearing gabbro (Gabbro 1) (Sample 3121256D-214R-2, 78-81 cm; 1413.13 mbsf). Network domain has distinct exsolution lamellae. B. Same as A (cross-polarized light). Clinopyroxene with exsolution lamellae replaces poikilitic clinopyroxene in subophitic domain. Clinopyroxenes of both domains are igneous-type. Plagioclase in the coarse-grained network domain shows normal zoning. C. Disseminated oxide gabbronorite (Gabbro 2), which consists of Fe-Ti oxide, clinopyroxene, orthopyroxene, and plagioclase with cloudy core and clear rim due to numerous submicrometer opaque inclusions (Sample 312-1256D-232R-2, 10-14 cm; $1494.08 \mathrm{mbsf}$ ). Both orthopyroxene and clinopyroxene are recrystallized to green and brown amphibole. D. Disseminated oxide gabbronorite (Gabbro 2) (Sample 312-1256D-231R-2, 95-98 cm, 1490.14 mbsf). Amphibole intergrowth-type clinopyroxenes are partially replaced by green and brown amphibole and Fe-Ti oxides a few to a few tens of micrometers in diameter. E. Area adjacent to D, same thin section. Igneous type clinopyroxene characterized by exsolution lamellae with submicrometer-sized opaque minerals. F. Type 8 metabasalt (lower dike screen), showing granular texture like oxide gabbronorite (Sample 312-1256D-233R-1, 4-7 cm; $1497.50 \mathrm{mbsf}$ ). Secondary clinopyroxene with very low $\mathrm{TiO}_{2}$ forms a cluster or vein. Small cluster is visible at the center right. G. Type 8 metabasalt (lower dike screen) (Sample 312-1256D-234R-1, 1-2 cm; $1502.50 \mathrm{mbsf}$ ). 0.5 to $1.0 \mathrm{~mm}$ size euhedral plagioclase laths and relict clinopyroxene are embedded in the matrix of microgranular secondary clinopyroxene, orthopyroxene, plagioclase and Fe-Ti oxides. Plagioclase lath has granular opaque and transparent inclusions a few micrometers in diameter. $\mathbf{H}$. Same as $\mathrm{G}$ (cross-polarized light). $\mathrm{pl}=$ plagioclase, $\mathrm{cpx}=$ clinopyroxene, $\mathrm{amp}=\mathrm{amphibole}, \mathrm{ox}=$ Fe-Ti oxide, opx = orthopyroxene. (Figure shown on next page.) 
Figure F1 (continued). (Caption shown on previous page.)

A

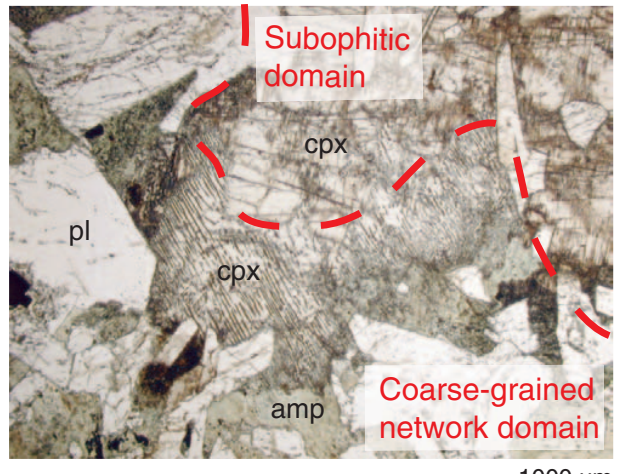

$1000 \mu \mathrm{m}$

C

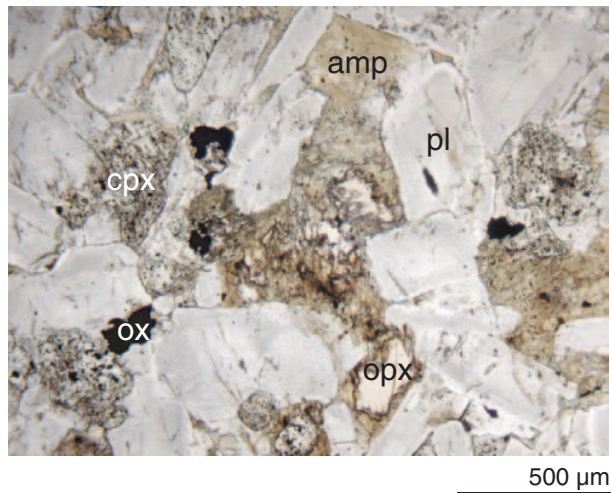

E

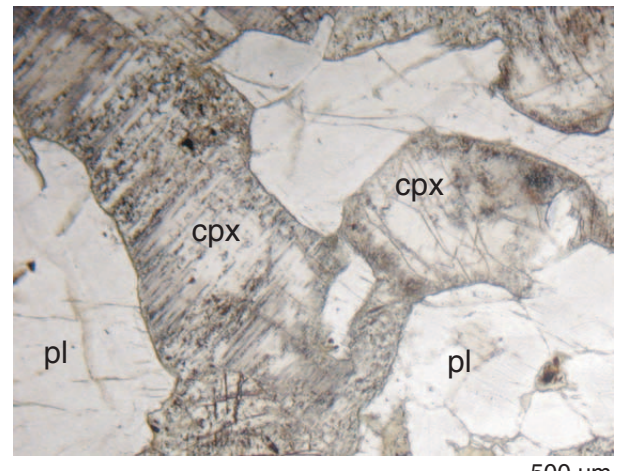

G

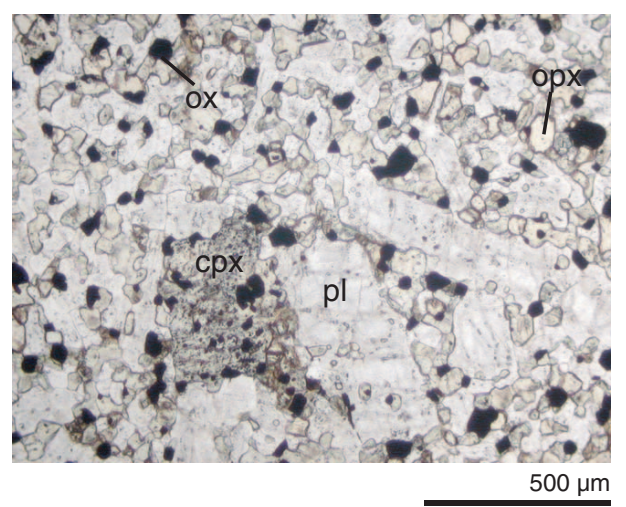

B

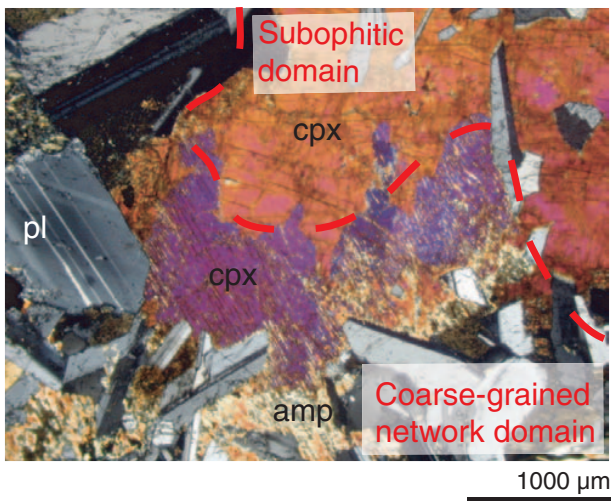

D

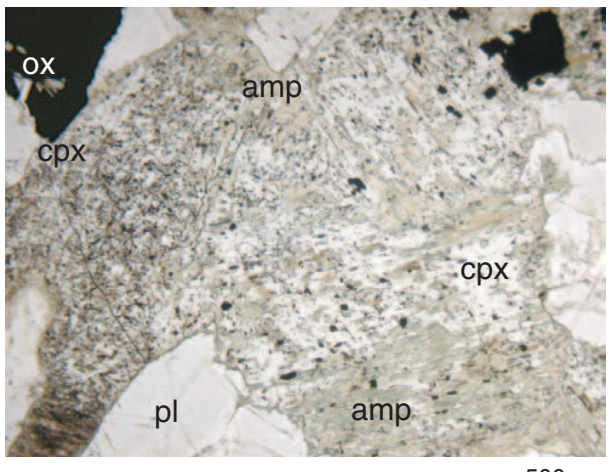

$500 \mu \mathrm{m}$

F

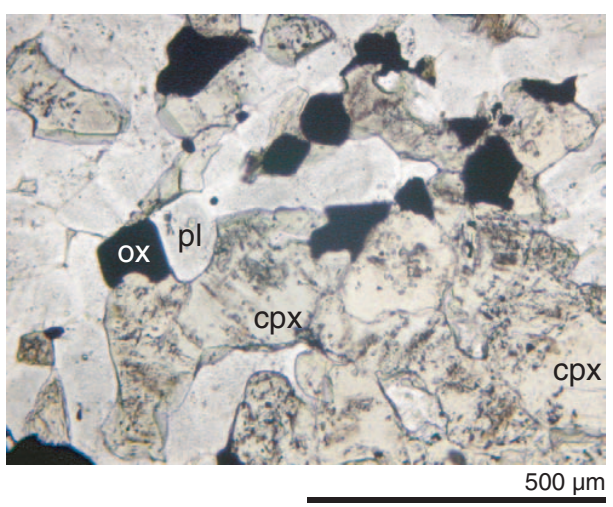

H

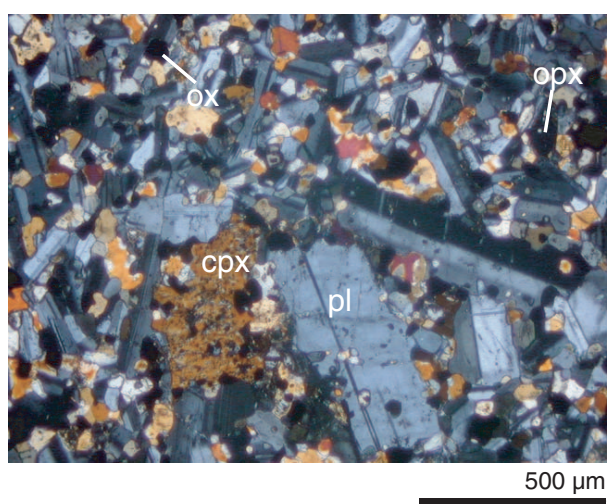


Figure F2. Images of typical heterogeneous texture of upper part of Gabbro 1, which is characterized by two textual domains: subophitic and coarse-grained patchy or network. A. Scan image of wet surface of oxide gabbro (Sample 312-1256D-214R-2, 78-81 cm; 1413.13 mbsf). White brackets $=$ area of thin section in B and C. B. Thin section image of A (cross-polarized light). Subophitic domains are characterized by $<1 \mathrm{~cm}$ clinopyroxene oikocrysts with plagioclase chadocrysts. Coarse-grained network domains are characterized by plagioclase and Fe-Ti oxide. C. Thin section image of A (plane-polarized light). Red dashed line = boundary between coarse-grained network domain and subophitic domain. Yellow box $=$ area of the photomicrographs in Figure F1A and F1B.

A

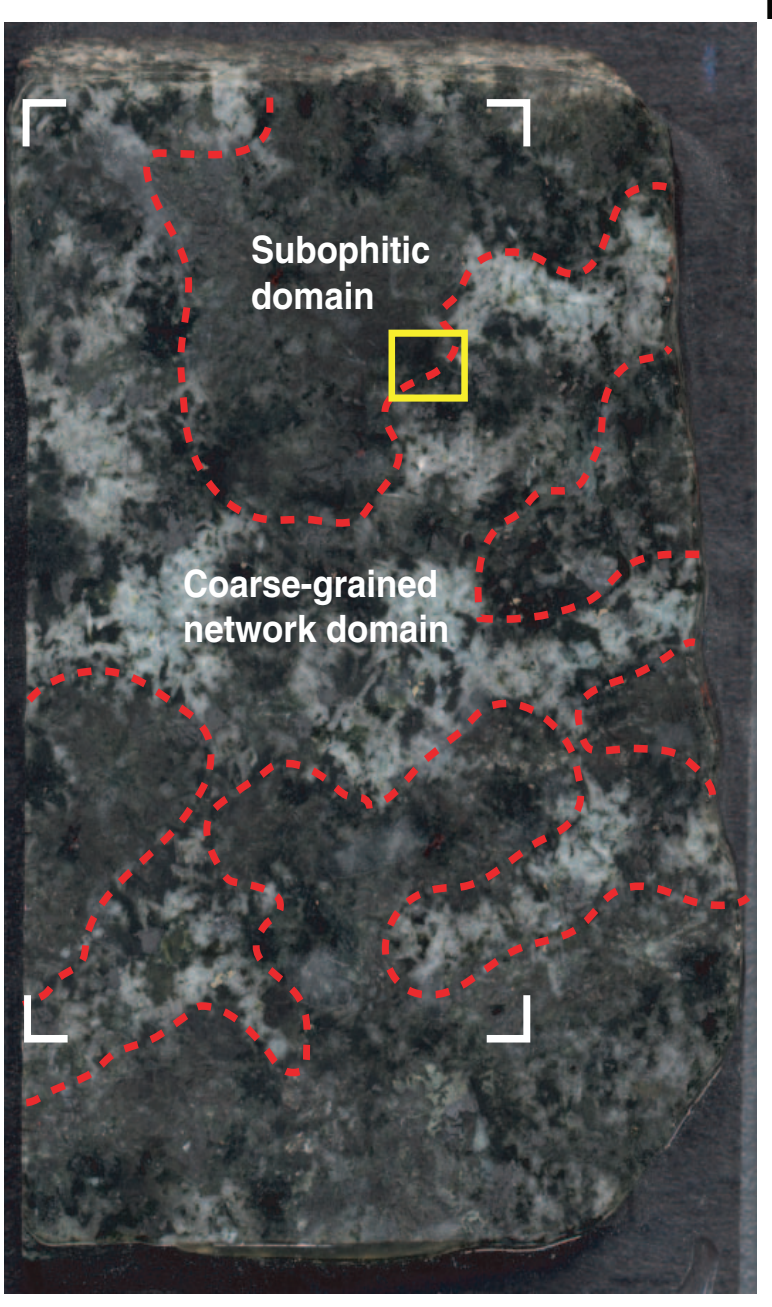

$1 \mathrm{~cm}$
B

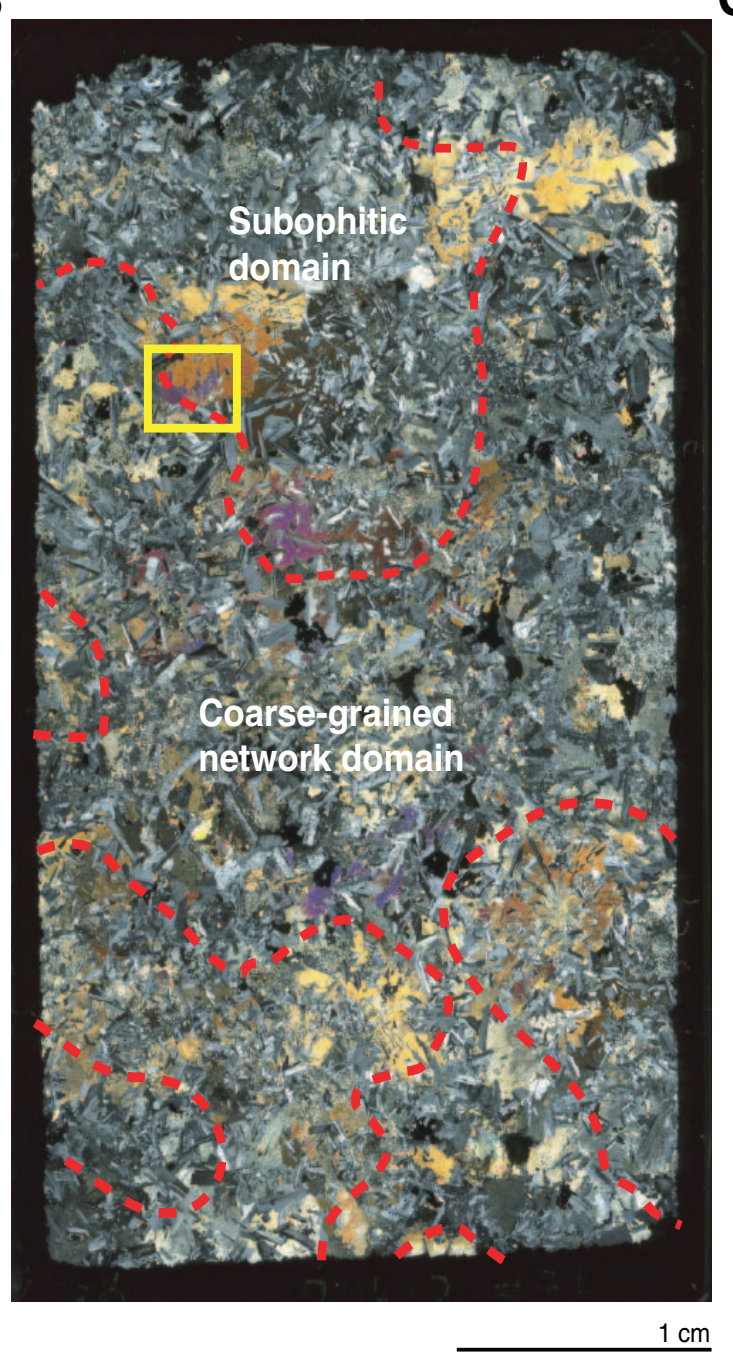

C

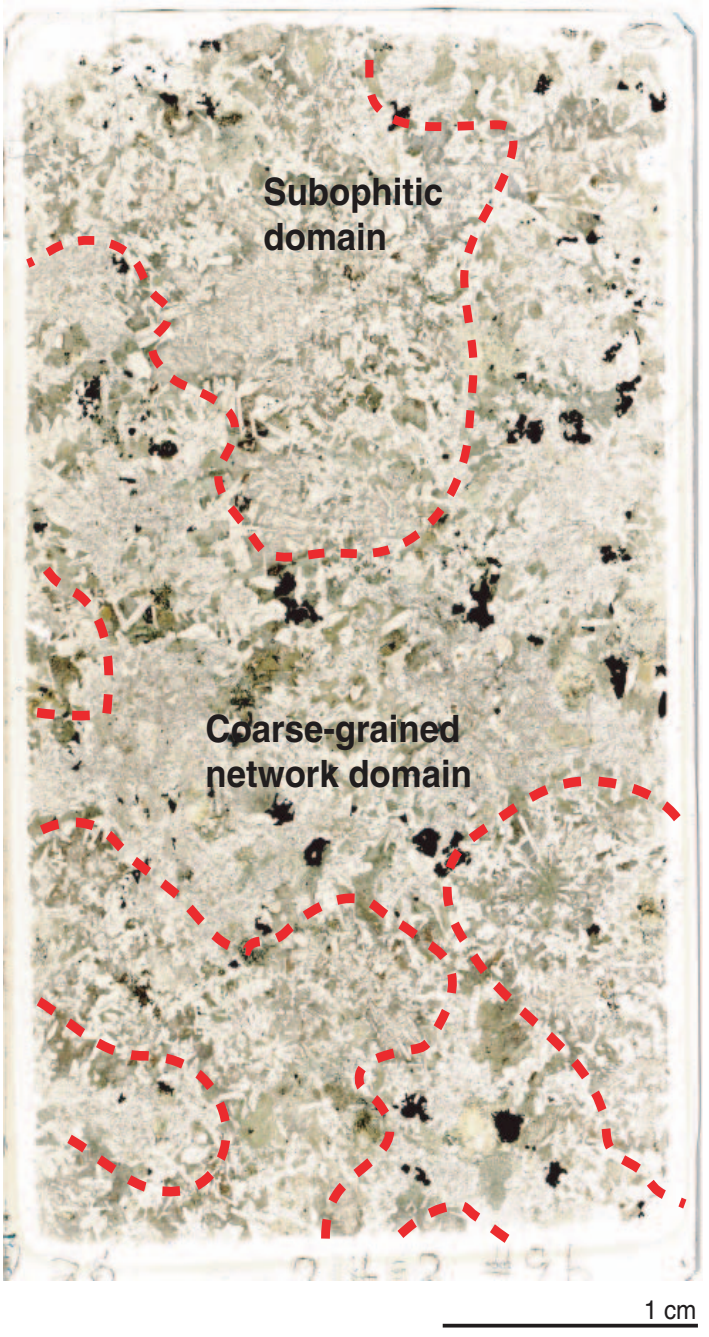


Figure F3. Variation diagrams of whole-rock compositions. A. $\mathrm{SiO}_{2}$ vs. $\mathrm{Mg \#}(100 \times \mathrm{Mg} /[\mathrm{Mg}+\mathrm{Fe}])$. B. Loss on ignition vs. $\mathrm{K}_{2} \mathrm{O}$. C. $\mathrm{Mg} \#$ vs. $\mathrm{TiO}_{2}$. D. $\mathrm{Mg} \#$ vs. $\mathrm{P}_{2} \mathrm{O}_{5}$.
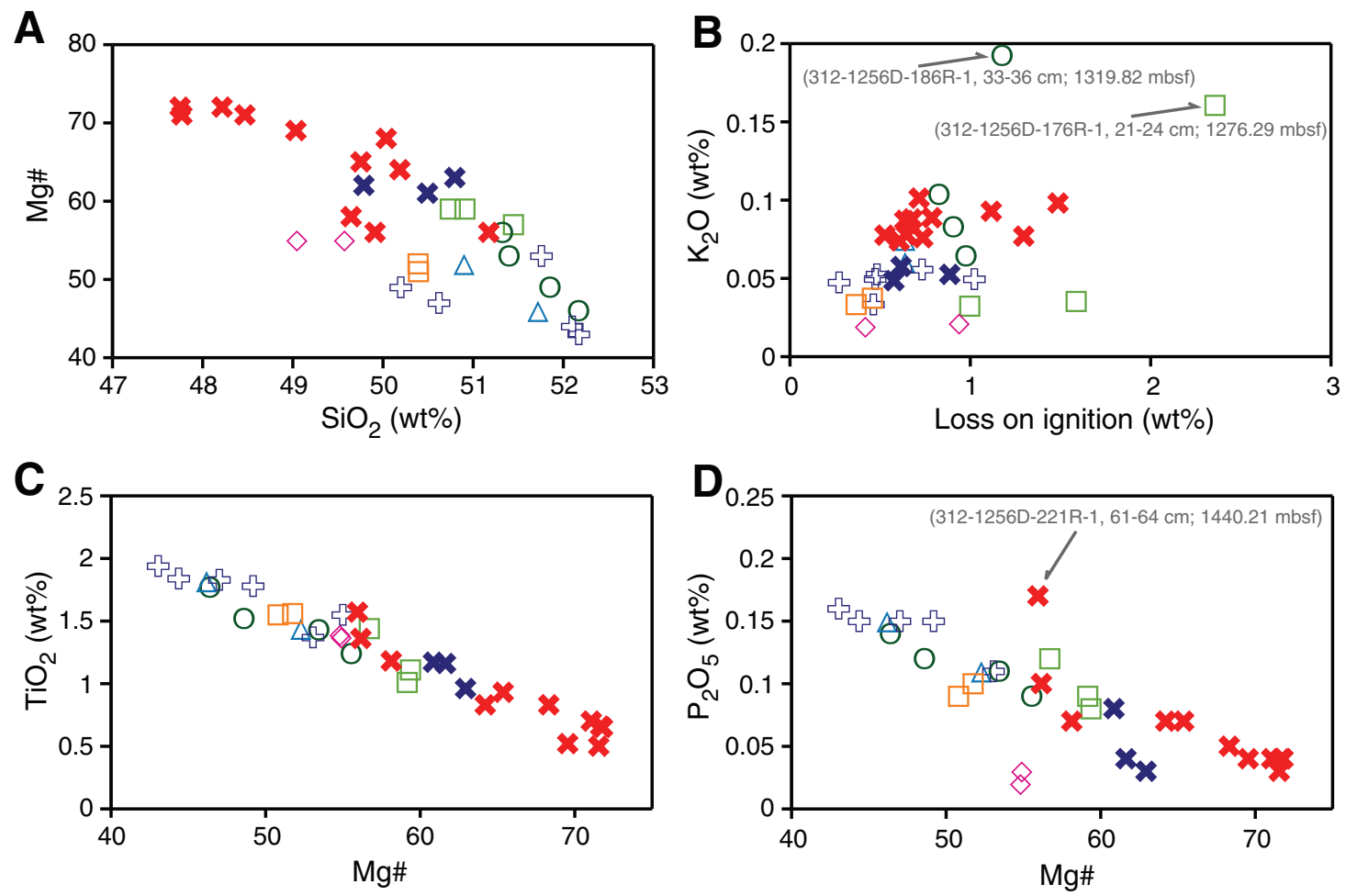

$\square$ Type 3 basalt

O Type 4 basalt

$\triangle$ Type 6 metabasalt ß Type 7 metabasalt

* Gabbro 1

$\square$ Upper dike screen (Type 8 metabasalt)

* Gabbro 2

$\diamond$ Lower dike screen (Type 8 metabasalt) 


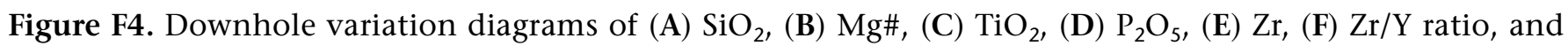
(G) $\mathrm{La} / \mathrm{Sm}$ ratio normalized to normal mid-ocean-ridge basalt values of Sun and McDonough (1989).
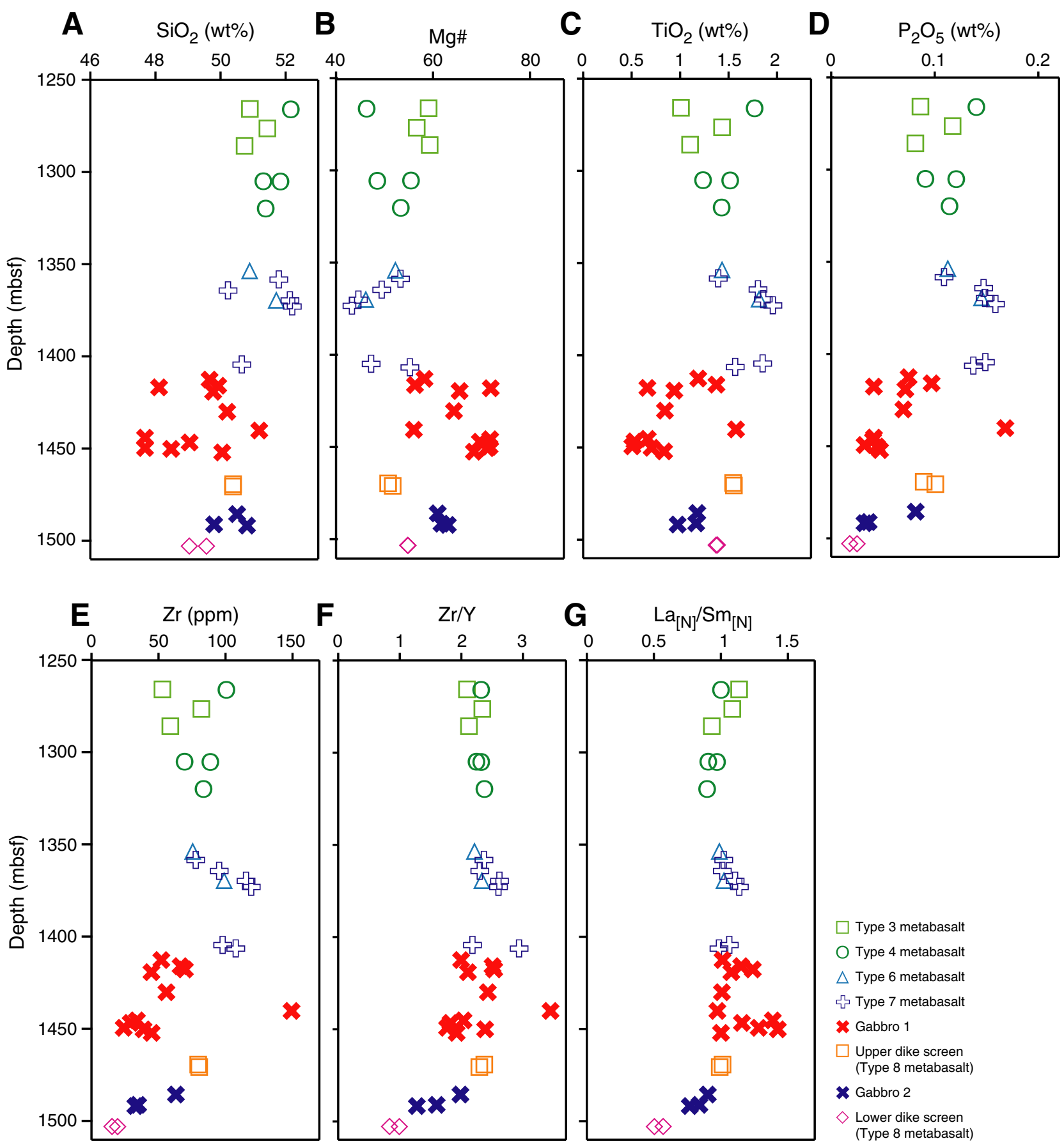
Figure F5. Trace element patterns normalized to normal mid-ocean-ridge basalt (N-MORB) values of Sun and McDonough (1989). A. Type 3 and 4 basalts and Type 6 and 7 metabasalts. B. Gabbro 1. C. Gabbro 2. D. Upper and lower dike screen (Type 8 metabasalts). Numeric value of symbol indicates sampling depth (meters below seafloor).
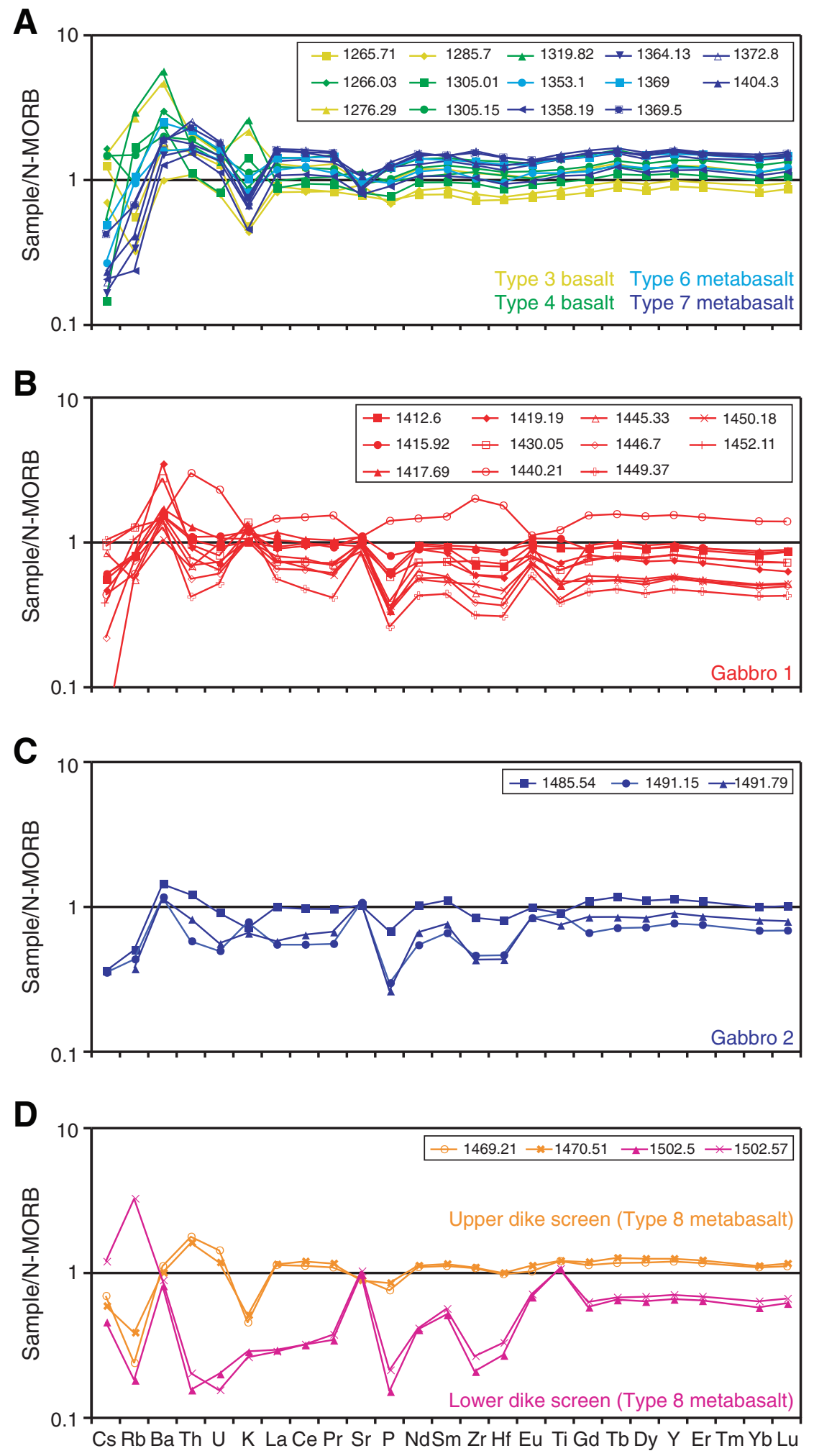
Figure F6. Normal mid-ocean-ridge basalt-normalized La/Sm ratio plotted against Zr.

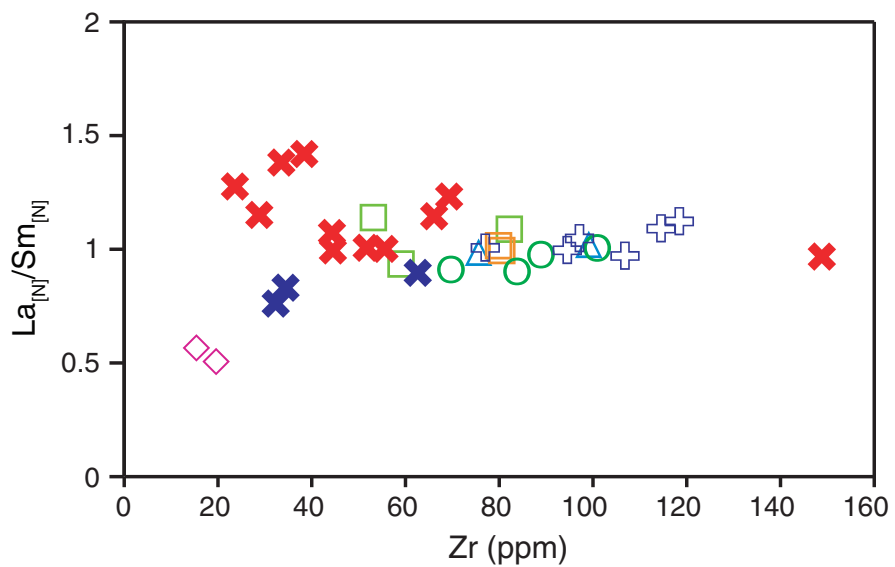

$\square$ Type 3 basalt

O Type 4 basalt

$\triangle$ Type 6 metabasalt

§ Type 7 metabasalt

* Gabbro 1

$\square$ Upper dike screen

(Type 8 metabasalt)

* Gabbro 2

$\diamond$ Lower dike screen (Type 8 metabasalt) 
Figure F7. Variation diagrams showing clinopyroxene $\mathrm{Mg \#}$ vs. $\mathrm{TiO}_{2}$ for (A) Type 3 to 7 basalts and metabasalts and the upper and lower dike screen, (B) Gabbros 1 and 2, and (C) subophitic and coarse-grained network domains of Gabbro 1.
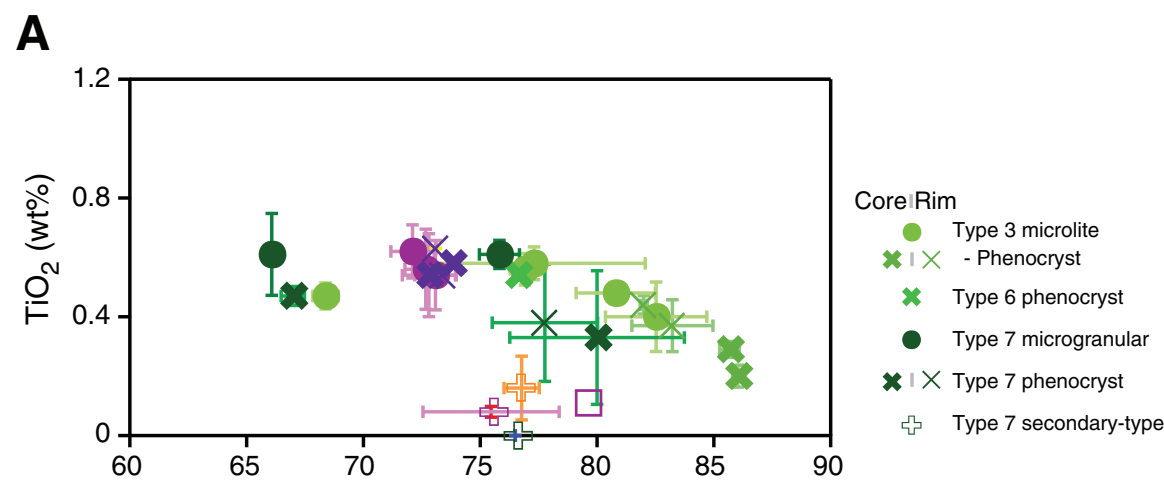

- Type 7 microgranular

* $1 \times$ Type 7 phenocryst

¡ Type 7 secondary-type

Core IRim

Upper dike screen secondary-type

Lower dike screen

- - Microgranular

$\square \quad$ - Amphibole-type

\& - Secondary-type

$* \mid \times$ - Phenocryst

B

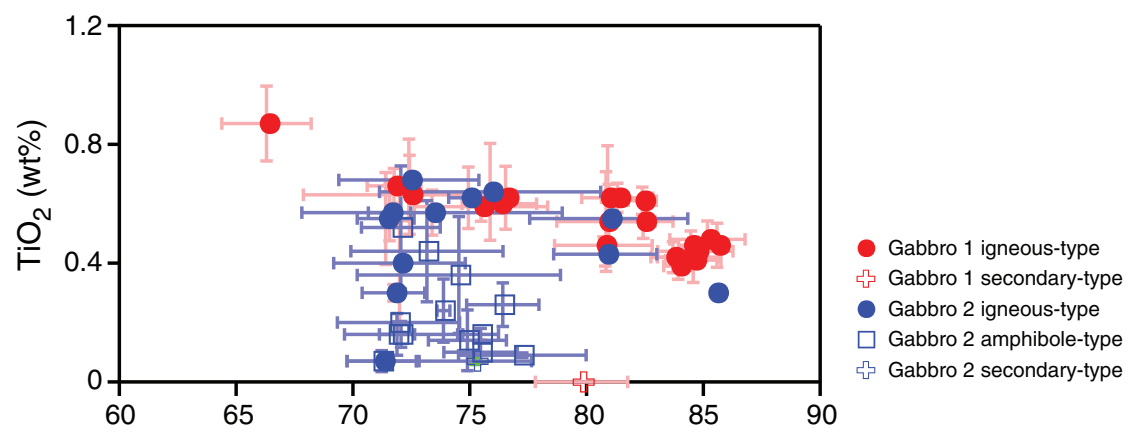

C

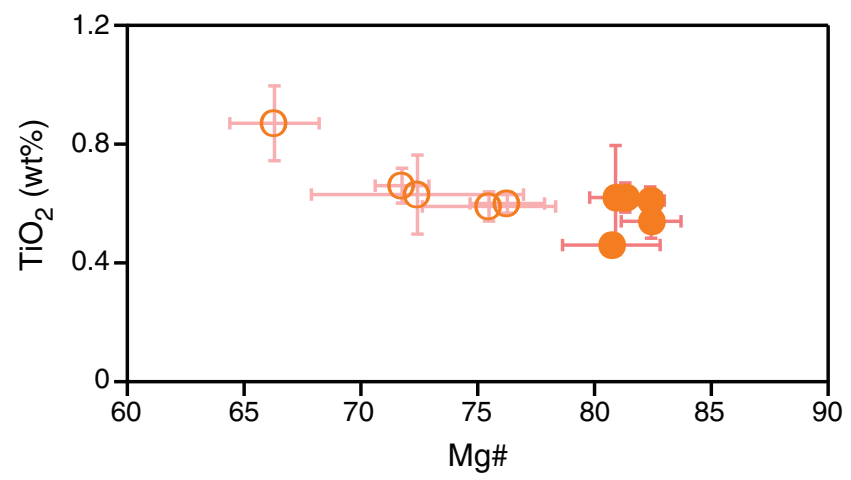

Gabbro 1 subophitic domain

Gabbro 1 coarse-grained
network domain 
Figure F8. Downhole variations showing clinopyroxene (Cpx) (A) $\mathrm{Mg \#} \mathrm{(B)} \mathrm{and} \mathrm{TiO}_{2}$, orthopyroxene $(\mathrm{Opx})(\mathbf{C}) \mathrm{Mg \#}$ and $(\mathbf{D}) \mathrm{TiO} \mathrm{O}_{2}$, plagioclase $(\mathrm{Pl})$ (E) anorthite (An) and (F) MgO, and (G) olivine (Ol) forsterite (Fo). Lower depth range = close-up of Gabbro 2 and lower dike screen. Green sections = basalts, blue sections = gabbros, gray spotted pattern = granoblastic dikes. Symbols for plagioclase, orthopyroxene, and olivine are the same as in Figure F9. Symbols for clinopyroxene are the same as in Figure F7.

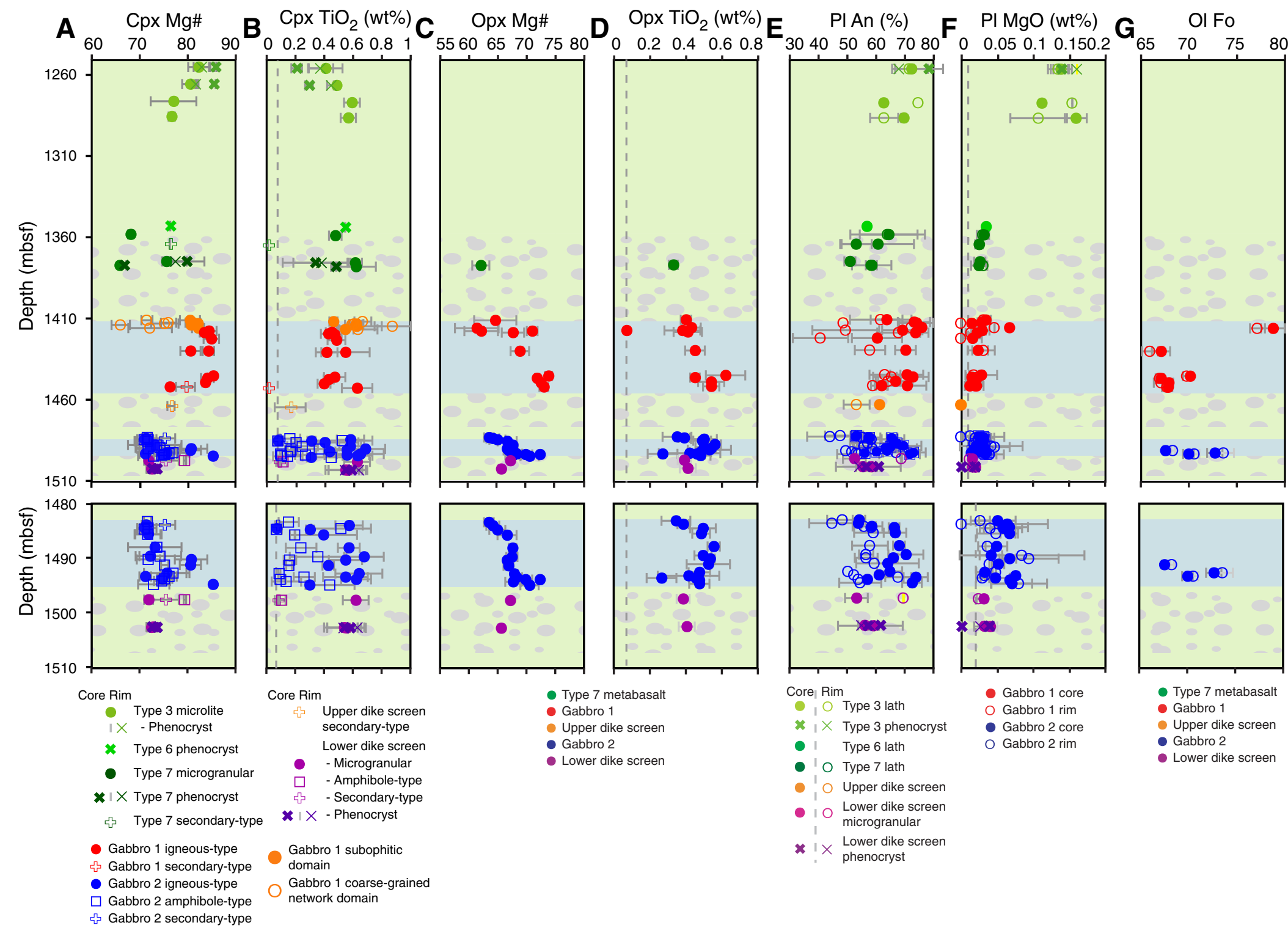


Figure F9. Mineral compositions of (A) orthopyroxene $\mathrm{Mg \#}$ vs. $\mathrm{TiO}_{2}$, (B) olivine forsterite (Fo) vs. NiO, and plagioclase anorthite (An) vs. (C) MgO of Type 3 to 8 basalts and metabasalts and (D) Gabbros 1 and 2.
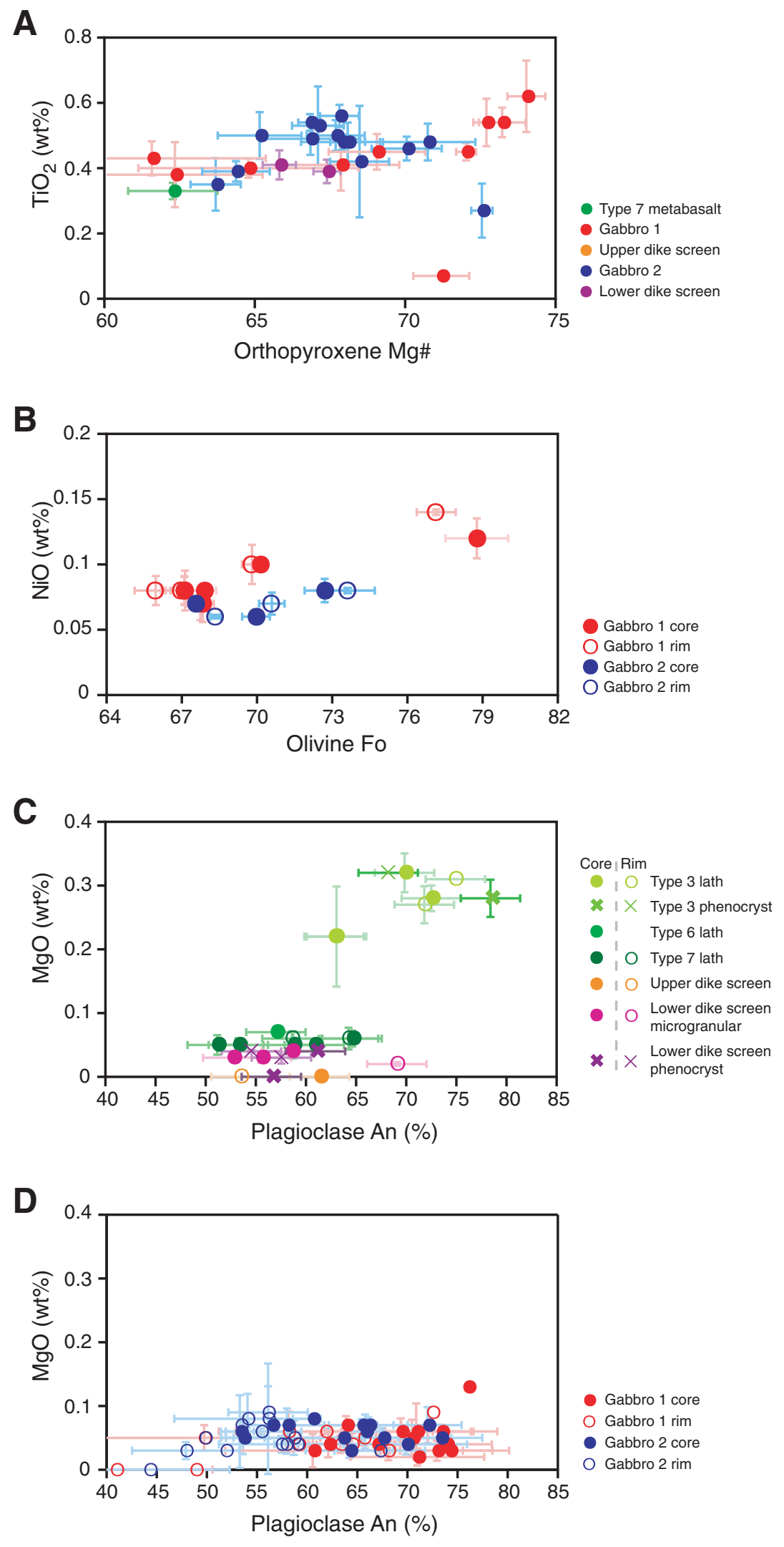
Figure F10. Olivine forsterite (Fo) content of core vs. rim. Gabbro 1 olivines plotted below the line are normally zoned; Gabbro 2 olivines are reversely zoned and plot above the line.

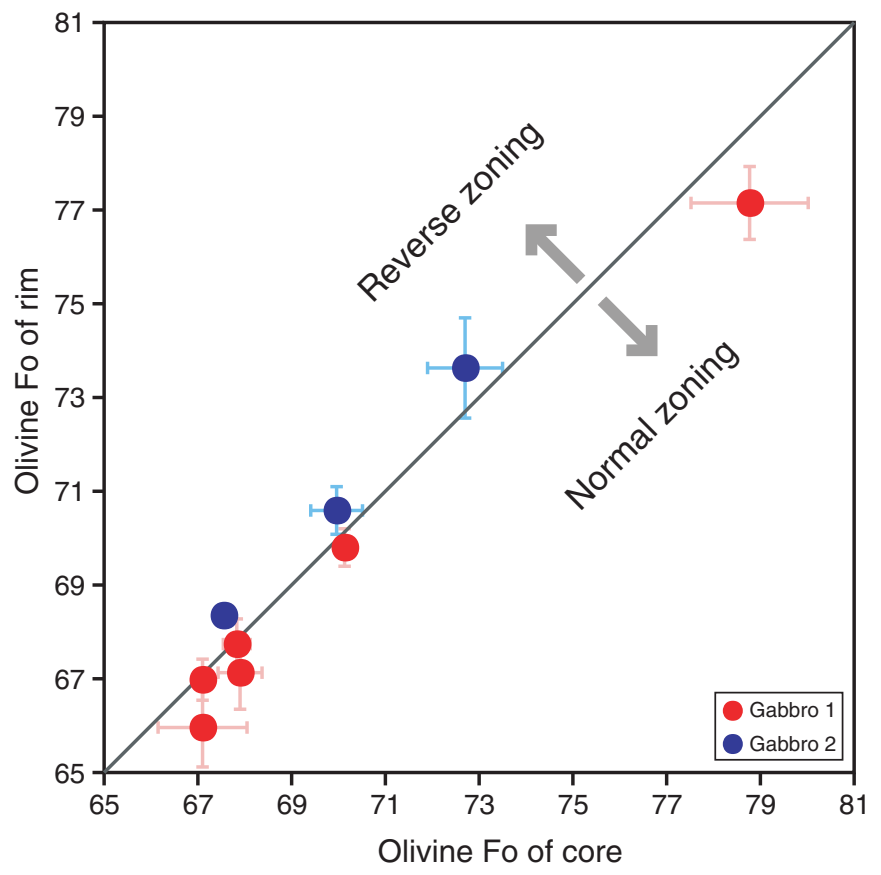


Table T1. Analyzed samples, Hole 1256D. (See table notes.) (Continued on next page.)

\begin{tabular}{|c|c|c|c|c|c|c|c|c|c|}
\hline $\begin{array}{l}\text { Core, section, } \\
\text { interval }(\mathrm{cm})\end{array}$ & $\begin{array}{l}\text { Depth } \\
\text { (mbsf) }\end{array}$ & Unit & Lithology & $\begin{array}{l}\text { Rock name and basalt } \\
\text { texture type }\end{array}$ & $\begin{array}{l}\text { Phenocryst } \\
\text { assemblages } \\
\text { for basalt }\end{array}$ & $\begin{array}{c}\text { Igneous mineral } \\
\text { assemblages for } \\
\text { gabbro }\end{array}$ & $\begin{array}{l}\text { Secondary mineral } \\
\text { assemblage }\end{array}$ & EPMA & $\begin{array}{l}\text { XRF and } \\
\text { ICP-MS }\end{array}$ \\
\hline \multicolumn{10}{|l|}{$312-1256 \mathrm{D}-$} \\
\hline 172R-1,12-14 & 1255.20 & 66 & Sheeted dike & Type 3 basalt & $\mathrm{Pl}, \mathrm{Cpx}$ & & DBM, Chl & $\mathrm{x}$ & \\
\hline $174 \mathrm{R}-1,32-34$ & 1265.71 & 68 & Sheeted dike & Type 3 basalt & $\mathrm{PI}, \mathrm{Cpx}$ & & DBM, Chl & $\mathrm{x}$ & $\mathrm{x}$ \\
\hline $174 \mathrm{R}-1,64-67$ & 1266.03 & 68 & Sheeted dike & Type 4 basalt & Aphyric & & DBM, Chl & & $\mathrm{x}$ \\
\hline $176 \mathrm{R}-1,21-24$ & 1276.29 & 69 & Sheeted dike & Type 3 basalt & $\mathrm{Pl}, \mathrm{Cpx}$ & & DBM, Chl & $\mathrm{x}$ & $\mathrm{x}$ \\
\hline $178 \mathrm{R}-1,0-3$ & 1285.70 & 73 & Sheeted dike & Type 3 basalt & $\mathrm{Pl}$ & & DBM, Chl & $\mathrm{x}$ & $x$ \\
\hline $182 \mathrm{R}-1,12-15$ & 1305.01 & 73 & Sheeted dike & Type 4 basalt & Aphyric & & DBM, Chl, Ox & & $\mathrm{x}$ \\
\hline 184R-1, 39-41 & 1305.15 & 74 & Sheeted dike & Type 4 basalt & $\mathrm{Pl}, \mathrm{Cpx}$ & & DBM, Chl, Ox & & $\mathrm{x}$ \\
\hline 186R-1, 33-36 & 1319.82 & $75 \mathrm{~A}$ & Sheeted dike & Type 4 basalt & Aphyric & & DBM, Chl, Ox, Amp & & $\mathrm{x}$ \\
\hline $193 R-1,5-8$ & 1353.10 & 78 & Granoblastic dike & Type 6 metabasalt & Aphyric & & $\mathrm{DBM}, \mathrm{Ox}, \mathrm{Amp}$ & $\mathrm{x}$ & $\mathrm{x}$ \\
\hline 194R-1, 29-33 & 1358.19 & 78 & Granoblastic dike & Type 7 metabasalt & $\mathrm{Pl}$ & & DBM, Ox, Amp, Opx & $\mathrm{x}$ & $\mathrm{x}$ \\
\hline 196R-1, 43-46 & 1364.13 & 78 & Granoblastic dike & Type 7 metabasalt & $\mathrm{Pl}$ & & Chl, Ox, Amp, Cpx, Opx & $\mathrm{x}$ & $\mathrm{x}$ \\
\hline $198 \mathrm{R}-1,0-5$ & 1369.00 & 78 & Granoblastic dike & Type 6 metabasalt & Aphyric & & DBM, Ox, Amp & & $\mathrm{x}$ \\
\hline $198 \mathrm{R}-1,50-54$ & 1369.50 & 78 & Granoblastic dike & Type 7 metabasalt & Aphyric & & $\begin{array}{l}\text { DBM, Chl, Ox, Amp, Cpx, } \\
\text { Opx }\end{array}$ & & $x$ \\
\hline $202 \mathrm{R}-1,5-8$ & 1372.80 & 79 & Granoblastic dike & Type 7 metabasalt & Aphyric & & DBM, Ox, Amp, Cpx, Opx & & $\mathrm{x}$ \\
\hline 203R-1, 6-10 & 1374.89 & $80 \mathrm{~A}$ & Granoblastic dike & Type 7 metabasalt & $\mathrm{Pl}, \mathrm{Cpx}$ & & Amp, Ox, Cpx, Opx, Qtz & $\mathrm{x}$ & \\
\hline 204R-1, 0-4 & 1377.30 & $80 \mathrm{~A}$ & Granoblastic dike & Type 7 metabasalt & Aphyric & & Amp, Ox, Cpx, Opx & $\mathrm{x}$ & \\
\hline $212 \mathrm{R}-1,20-23$ & 1404.30 & $80 \mathrm{~A}$ & Granoblastic dike & Type 7 metabasalt & Aphyric & & Chl, Amp, Ox, Cpx, Opx & & $x$ \\
\hline $214 \mathrm{R}-1,19-21$ & 1411.10 & 81 & Gabbro 1 & $\begin{array}{l}\text { Opx-bearing ol } \\
\text { gabbro }\end{array}$ & & $\mathrm{Pl}, \mathrm{Cpx}, \mathrm{Ol}, \mathrm{Opx}, \mathrm{Ox}$ & Amp, Tlc, Id & $x$ & \\
\hline $214 \mathrm{R}-2,24-27$ & 1412.60 & 83 & Gabbro 1 & $\begin{array}{l}\text { Disseminated oxide } \\
\text { gabbro }\end{array}$ & & $\mathrm{PI}, \mathrm{Cpx}, \mathrm{Ox}$ & Chl, Amp, Ox & $x$ & $x$ \\
\hline $214 \mathrm{R}-2,78-81$ & 1413.13 & 85 & Gabbro 1 & $\begin{array}{l}\text { Disseminated oxide } \\
\text { gabbro }\end{array}$ & & $\mathrm{Pl}, \mathrm{Cpx}, \mathrm{Ol}, \mathrm{Ox}$ & Chl, Amp, Ox, Ep, Id & $x$ & \\
\hline $214 R-3,18-21$ & 1413.99 & 85 & Gabbro 1 & $\begin{array}{l}\text { Disseminated oxide ol } \\
\text { gabbro }\end{array}$ & & $\mathrm{Pl}, \mathrm{Cpx}, \mathrm{Ox}$ & Amp, Ox, Id, Ab & $x$ & \\
\hline $215 R-1,20-23$ & 1415.92 & 85 & Gabbro 1 & $\begin{array}{l}\text { Disseminated oxide ol } \\
\text { gabbro }\end{array}$ & & $\mathrm{Pl}, \mathrm{Cpx}, \mathrm{Ol}, \mathrm{Opx}, \mathrm{Ox}$ & Amp, Chl, Ox, Ep, Id, Ab & $x$ & $x$ \\
\hline $215 R-2,56-59$ & 1417.69 & $86 \mathrm{~A}$ & Gabbro 1 & $\begin{array}{l}\text { Disseminated oxide ol } \\
\text { gabbro }\end{array}$ & & $\mathrm{Pl}, \mathrm{Cpx}, \mathrm{Ox}$ & Amp, Ox, Id, Ab & $x$ & $x$ \\
\hline $216 \mathrm{R}-1,72-75$ & 1418.62 & $86 \mathrm{~A}$ & Gabbro 1 & Ol gabbro & & $\mathrm{Pl}, \mathrm{Cpx}$ & Amp, Ox, Id, Tlc & $\mathrm{x}$ & \\
\hline $216 \mathrm{R}-1,138-142$ & 1419.19 & 86 & Gabbro 1 & Ol gabbro & & $\mathrm{Pl}, \mathrm{Cpx}$ & Chl, Amp, Ox & $\mathrm{x}$ & $\mathrm{x}$ \\
\hline 217R-1, 94-97 & 1422.54 & 88 & Gabbro 1 & Gabbro & & $\mathrm{Pl}, \mathrm{Cpx}$ & Amp, Ox, Id, Ab & $\mathrm{x}$ & \\
\hline $218 \mathrm{R}-1,1-3$ & 1430.00 & 88 & Gabbro 1 & Gabbro & & $\mathrm{Pl}, \mathrm{Cpx}$ & Amp, Ox, Cal, Zeo, Ant, Ab & $\mathrm{x}$ & \\
\hline $219 R-1,5-8$ & 1430.05 & 88 & Gabbro 1 & $\begin{array}{l}\text { Opx-bearing ol } \\
\text { gabbro }\end{array}$ & & $\mathrm{Pl}, \mathrm{Cpx}, \mathrm{Ol}, \mathrm{Opx}$ & Amp, Ox, Id & $x$ & $x$ \\
\hline $221 \mathrm{R}-1,61-64$ & 1440.21 & 88 & Gabbro 1 & Opx-bearing gabbro & & $\mathrm{PI}, \mathrm{Cpx}$ & $A m p, O x, A b$ & & $\mathrm{x}$ \\
\hline $222 \mathrm{R}-1,73-78$ & 1445.33 & 88 & Gabbro 1 & $\begin{array}{l}\text { Opx-bearing ol } \\
\text { gabbro }\end{array}$ & & $\mathrm{PI}, \mathrm{Cpx}, \mathrm{OI}$ & Amp, Ox, Id, Tlc & $x$ & $x$ \\
\hline $222 \mathrm{R}-2,60-63$ & 1446.70 & 88 & Gabbro 1 & $\begin{array}{l}\text { Opx-bearing ol } \\
\text { gabbro }\end{array}$ & & $\mathrm{Pl}, \mathrm{Cpx}, \mathrm{Ol}, \mathrm{Opx}$ & Amp, Ox, Id & $x$ & $x$ \\
\hline $223 R-1,8-12$ & 1449.37 & 88 & Gabbro 1 & $\begin{array}{l}\text { Opx-bearing ol } \\
\text { gabbro }\end{array}$ & & $\mathrm{Pl}, \mathrm{Cpx}, \mathrm{Ol}, \mathrm{Opx}$ & Amp, Ox, Id, Cal & $x$ & $x$ \\
\hline 223R-1, 88-91 & 1450.18 & 88 & Gabbro 1 & $\begin{array}{l}\text { Opx-bearing ol } \\
\text { gabbro }\end{array}$ & & $\mathrm{Pl}, \mathrm{Cpx}, \mathrm{Ol}, \mathrm{Opx}$ & Amp, Ox, Id, Tlc & & $x$ \\
\hline 223R-2, 133-137 & 1452.11 & $89 \mathrm{~A}$ & Gabbro 1 & Ol gabbronorite & & $\mathrm{PI}, \mathrm{Cpx}, \mathrm{Ol}, \mathrm{Opx}, \mathrm{Ox}$ & Amp, Ox & $\mathrm{x}$ & $\mathrm{x}$ \\
\hline $226 \mathrm{R}-1,0-4$ & 1463.90 & $90 \mathrm{~A}$ & Upper dike screen & Type 8 metabasalt & Aphyric & & Amp, Ox, Cpx, Opx & $\mathrm{x}$ & \\
\hline 227R-1, 72-76 & 1469.21 & $90 \mathrm{~A}$ & Upper dike screen & Type 8 metabasalt & Aphyric & & Amp, Ox, Cpx, Opx & & $\mathrm{x}$ \\
\hline 227R-2, 50-59 & 1470.51 & $90 \mathrm{~A}$ & Upper dike screen & Type 8 metabasalt & Aphyric & & Amp, Ox, Cpx, Opx & & $\mathrm{x}$ \\
\hline 230R-1, 19-21 & 1483.19 & $90 \mathrm{~A}$ & Gabbro 2 & $\begin{array}{l}\text { Disseminated oxide } \\
\text { opx-bearing } \\
\text { gabbro }\end{array}$ & & $\mathrm{Pl}, \mathrm{Cpx}, \mathrm{Opx}, \mathrm{Ox}$ & Amp, Ox & $\mathrm{x}$ & \\
\hline 230R-1, 81-84 & 1483.81 & $91 \mathrm{~A}$ & Gabbro 2 & $\begin{array}{l}\text { Disseminated oxide } \\
\text { gabbro }\end{array}$ & & $\mathrm{Pl}, \mathrm{Cpx}, \mathrm{Opx}, \mathrm{Ox}$ & Amp, Ox & $x$ & \\
\hline 230R-1, 139-142 & 1484.38 & $92 \mathrm{~A}$ & Gabbro 2 & Gabbronorite & & $\mathrm{Pl}, \mathrm{Cpx}, \mathrm{Opx}$ & Amp, Ox & $x$ & \\
\hline $230 \mathrm{R}-2,32-36$ & 1484.58 & $92 \mathrm{~A}$ & Gabbro 2 & Gabbronorite & & $\mathrm{Pl}, \mathrm{Cpx}, \mathrm{Opx}$ & Amp, Ox & $\mathrm{x}$ & \\
\hline 230R-2, 104-109 & 1485.54 & $92 \mathrm{~A}$ & Gabbro 2 & Gabbronorite & & $\mathrm{Pl}, \mathrm{Cpx}, \mathrm{Opx}, \mathrm{Ox}$ & Amp, Ox & $\mathrm{x}$ & $\mathrm{x}$ \\
\hline $231 \mathrm{R}-1,19-22$ & 1487.90 & $92 \mathrm{~A}$ & Gabbro 2 & $\begin{array}{l}\text { Disseminated oxide } \\
\text { gabbronorite }\end{array}$ & & $\mathrm{Pl}, \mathrm{Cpx}, \mathrm{Opx}, \mathrm{Ox}$ & Amp, Ox & $x$ & \\
\hline $231 \mathrm{R}-2,35-39$ & 1489.54 & $92 \mathrm{~A}$ & Gabbro 2 & $\begin{array}{l}\text { Disseminated oxide } \\
\text { gabbronorite }\end{array}$ & & $\mathrm{Pl}, \mathrm{Cpx}, \mathrm{Opx}, \mathrm{Ox}$ & Amp, Ox & $x$ & \\
\hline $231 \mathrm{R}-2,95-98$ & 1490.14 & $92 \mathrm{~A}$ & Gabbro 2 & $\begin{array}{l}\text { Disseminated oxide } \\
\text { gabbronorite }\end{array}$ & & $\mathrm{Pl}, \mathrm{Cpx}, \mathrm{Opx}, \mathrm{Ox}$ & Amp, Ox, Id & $x$ & \\
\hline $231 \mathrm{R}-3,59-63$ & 1491.15 & $92 \mathrm{~A}$ & Gabbro 2 & $\begin{array}{l}\text { Disseminated oxide } \\
\text { ol-bearing } \\
\text { gabbronorite }\end{array}$ & & $\mathrm{Pl}, \mathrm{Cpx}, \mathrm{Ol}, \mathrm{Opx}, \mathrm{Ox}$ & Amp, Ox, Id, Tlc & $x$ & $x$ \\
\hline $231 \mathrm{R}-3,123-127$ & 1491.79 & $92 \mathrm{~A}$ & Gabbro 2 & $\begin{array}{l}\text { Disseminated oxide } \\
\text { gabbronorite }\end{array}$ & & $\mathrm{Pl}, \mathrm{Cpx}, \mathrm{Opx}, \mathrm{Ox}$ & Amp, Ox, Id, Tlc & & $x$ \\
\hline
\end{tabular}


Table T1 (continued).

\begin{tabular}{|c|c|c|c|c|c|c|c|c|c|}
\hline $\begin{array}{l}\text { Core, section, } \\
\text { interval }(\mathrm{cm})\end{array}$ & $\begin{array}{l}\text { Depth } \\
\text { (mbsf) }\end{array}$ & Unit & Lithology & $\begin{array}{l}\text { Rock name and basalt } \\
\text { texture type }\end{array}$ & $\begin{array}{l}\text { Phenocryst } \\
\text { assemblages } \\
\text { for basalt }\end{array}$ & $\begin{array}{c}\text { Igneous mineral } \\
\text { assemblages for } \\
\text { gabbro }\end{array}$ & $\begin{array}{l}\text { Secondary mineral } \\
\text { assemblage }\end{array}$ & EPMA & $\begin{array}{l}\text { XRF and } \\
\text { ICP-MS }\end{array}$ \\
\hline $231 \mathrm{R}-4,70-74$ & 1492.63 & $92 \mathrm{~A}$ & Gabbro 2 & $\begin{array}{l}\text { Opx-bearing ol } \\
\text { gabbro }\end{array}$ & & $\mathrm{PI}, \mathrm{Cpx}, \mathrm{Ol}, \mathrm{Opx}, \mathrm{Ox}$ & Amp, Ox, Id, Tlc & $x$ & \\
\hline 231R-4, 137-141 & 1493.30 & $92 \mathrm{~A}$ & Gabbro 2 & Ol gabbronorite & & $\mathrm{Pl}, \mathrm{Cpx}, \mathrm{Opx}, \mathrm{Ox}$ & Amp, Ox & & \\
\hline $232 \mathrm{R}-1,36-39$ & 1493.26 & $92 \mathrm{~A}$ & Gabbro 2 & $\begin{array}{l}\text { Disseminated oxide } \\
\text { gabbronorite }\end{array}$ & & $\mathrm{Pl}, \mathrm{Cpx}, \mathrm{OI}, \mathrm{Opx}, \mathrm{Ox}$ & Amp, Ox, Id, Tlc & $x$ & \\
\hline $232 \mathrm{R}-1,78-82$ & 1493.68 & $92 \mathrm{~A}$ & Gabbro 2 & $\begin{array}{l}\text { Disseminated oxide } \\
\text { gabbronorite }\end{array}$ & & $\mathrm{Pl}, \mathrm{Cpx}, \mathrm{Opx}, \mathrm{Ox}$ & Amp, Ox, Id & $x$ & \\
\hline $232 \mathrm{R}-2,10-14$ & 1494.08 & $92 \mathrm{~A}$ & Gabbro 2 & $\begin{array}{l}\text { Disseminated oxide } \\
\text { gabbronorite }\end{array}$ & & $\mathrm{Pl}, \mathrm{Cpx}, \mathrm{Opx}, \mathrm{Ox}$ & Amp, Ox, Id & $x$ & \\
\hline 232R-2, 73-76 & 1494.71 & 93 & Gabbro 2 & $\begin{array}{l}\text { Disseminated oxide ol } \\
\text { gabbronorite }\end{array}$ & & $\mathrm{Pl}, \mathrm{Cpx}, \mathrm{Opx}, \mathrm{Ox}$ & Amp, Ox, Id & $x$ & \\
\hline 233R-1, 4-7 & 1497.50 & 94 & Lower dike screen & Type 8 metabasalt & Cpx & & Cpx, Opx, Pl, Ox, Amp & $x$ & \\
\hline $234 \mathrm{R}-1,1-2$ & 1502.50 & 94 & Lower dike screen & Type 8 metabasalt & $\mathrm{Pl}$ & & Cpx, Opx, PI, Ox, Amp & $x$ & $x$ \\
\hline $234 \mathrm{R}-1,7-9$ & 1502.57 & 94 & Lower dike screen & Type 8 metabasalt & $\mathrm{Pl}$ & & Cpx, Opx, PI, Ox, Amp & $x$ & $x$ \\
\hline
\end{tabular}

Notes: For basalt texure type, basalt is classified according to Koepke et al. (2008) and the "Site 1256" chapter. EPMA = JXA-8600SX EPMA at the Graduate School of Science and Technology, Niigata University. XRF = X-ray fluorescence, ICP-MS = inductively coupled plasma-mass spectrometry. Opx = orthopyroxene, $\mathrm{Ol}=$ olivine, $\mathrm{Pl}=$ plagioclase, $\mathrm{Cpx}=$ clinopyroxene, $\mathrm{Ox}=\mathrm{Fe}-\mathrm{Ti}$ oxide, $\mathrm{DBM}=$ dusty brown material, $\mathrm{Chl}=$ chlorite, $\mathrm{Amp}=$ amphibole, $\mathrm{Qtz}=$ quartz, $\mathrm{Tlc}=$ talc, Id = iddingsite, $\mathrm{Ep}=$ epidote, $\mathrm{Ab}=$ albite, $\mathrm{Zeo}=$ zeolite, Ant = anthophyllite, Cal $=$ calcite . 
Table T2. Whole-rock major and trace elements, Hole 1256D. (See table notes.) (Continued on next two pages.)

\begin{tabular}{|c|c|c|c|c|c|c|c|c|c|c|c|c|c|c|c|c|c|}
\hline Core, section: & & & & 174R-1 & $174 \mathrm{R}-1$ & 176R-1 & 178R-1 & $182 \mathrm{R}-1$ & 184R-1 & 186R-1 & 193R-1 & 194R-1 & 196R-1 & 198R-1 & 198R-1 & $202 \mathrm{R}-1$ & $212 \mathrm{R}-1$ \\
\hline Interval $(\mathrm{cm})$ : & & & & $32-34$ & $64-67$ & $21-24$ & $0-3$ & $12-15$ & $39-41$ & $33-36$ & $5-8$ & $29-33$ & $43-46$ & $0-5$ & $50-54$ & $5-8$ & $20-23$ \\
\hline Depth (mbsf): & & & & 1265.71 & 1266.03 & 1276.29 & 1285.70 & 1305.01 & 1305.15 & 1319.82 & 1353.10 & 1358.19 & 1364.13 & 1369.00 & 1369.50 & 1372.80 & 1404.30 \\
\hline Lithology: & & & & SD & SD & SD & SD & SD & SD & SD & $G D$ & $G D$ & $G D$ & $G D$ & $G D$ & GD & $G D$ \\
\hline Rock name & $\begin{array}{l}\text { Reference } \\
\text { value }\end{array}$ & $\begin{array}{l}\text { Average } \\
(N=4)\end{array}$ & $\begin{array}{l}\text { Standard } \\
\text { deviation }\end{array}$ & $\begin{array}{l}\text { Type } 3 \\
\text { basalt }\end{array}$ & $\begin{array}{l}\text { Type } 4 \\
\text { basalt }\end{array}$ & $\begin{array}{l}\text { Type } 3 \\
\text { basalt }\end{array}$ & $\begin{array}{l}\text { Type } 3 \\
\text { basalt }\end{array}$ & $\begin{array}{l}\text { Type } 4 \\
\text { basalt }\end{array}$ & $\begin{array}{l}\text { Type } 4 \\
\text { basalt }\end{array}$ & $\begin{array}{l}\text { Type } 4 \\
\text { basalt }\end{array}$ & $\begin{array}{c}\text { Type } 6 \\
\text { metabasalt }\end{array}$ & $\begin{array}{c}\text { Type } 7 \\
\text { metabasalt }\end{array}$ & $\begin{array}{c}\text { Type } 7 \\
\text { metabasalt }\end{array}$ & $\begin{array}{c}\text { Type } 6 \\
\text { metabasalt }\end{array}$ & $\begin{array}{c}\text { Type } 7 \\
\text { metabasalt }\end{array}$ & $\begin{array}{c}\text { Type } 7 \\
\text { metabasalt }\end{array}$ & $\begin{array}{c}\text { Type } 7 \\
\text { metabasalt }\end{array}$ \\
\hline \multicolumn{18}{|c|}{ Major element oxide (wt\%): } \\
\hline $\mathrm{SiO}_{2}$ & 53.71 & 53.77 & 0.15 & 50.91 & 52.18 & 51.45 & 50.75 & 51.33 & 51.86 & 51.41 & 50.90 & 51.73 & 50.17 & 51.72 & 52.07 & 52.15 & 50.60 \\
\hline $\mathrm{TiO}_{2}$ & 1.20 & 1.20 & 0.00 & 1.00 & 1.77 & 1.42 & 1.10 & 1.24 & 1.51 & 1.43 & 1.44 & 1.37 & 1.79 & 1.81 & 1.83 & 1.94 & 1.83 \\
\hline $\mathrm{Al}_{2} \mathrm{O}_{3}$ & 14.77 & 14.77 & 0.03 & 14.46 & 13.09 & 13.19 & 14.40 & 13.94 & 13.26 & 13.48 & 13.31 & 13.77 & 13.51 & 13.10 & 13.18 & 12.95 & 13.23 \\
\hline $\mathrm{FeO}^{\text {total }}$ & 12.95 & 12.92 & 0.01 & 10.02 & 13.46 & 10.97 & 10.03 & 11.35 & 12.88 & 11.93 & 12.81 & 11.68 & 13.50 & 13.72 & 14.01 & 14.26 & 13.97 \\
\hline $\mathrm{MnO}$ & 0.22 & 0.22 & 0.00 & 0.22 & 0.28 & 0.30 & 0.18 & 0.22 & 0.22 & 0.20 & 0.18 & 0.19 & 0.18 & 0.23 & 0.23 & 0.23 & 0.18 \\
\hline $\mathrm{MgO}$ & 4.66 & 4.63 & 0.06 & 8.15 & 6.55 & 8.07 & 8.24 & 7.97 & 6.84 & 7.70 & 7.88 & 7.36 & 7.29 & 6.61 & 6.23 & 6.01 & 6.91 \\
\hline $\mathrm{CaO}$ & 9.91 & 9.91 & 0.04 & 12.08 & 10.04 & 11.24 & 12.22 & 11.29 & 10.06 & 9.76 & 10.45 & 11.16 & 10.73 & 9.77 & 9.55 & 9.77 & 9.83 \\
\hline $\mathrm{Na}_{2} \mathrm{O}$ & 2.06 & 2.02 & 0.01 & 1.82 & 2.39 & 2.24 & 2.22 & 2.25 & 2.56 & 3.20 & 2.82 & 2.33 & 2.89 & 2.69 & 2.24 & 2.41 & 2.91 \\
\hline $\mathrm{K}_{2} \mathrm{O}$ & 0.43 & 0.43 & 0.01 & 0.03 & 0.06 & 0.16 & 0.03 & 0.10 & 0.08 & 0.19 & 0.06 & 0.03 & 0.05 & 0.07 & 0.06 & 0.05 & 0.05 \\
\hline $\mathrm{P}_{2} \mathrm{O}_{5}$ & 0.10 & 0.10 & 0.00 & 0.09 & 0.14 & 0.12 & 0.08 & 0.09 & 0.12 & 0.11 & 0.11 & 0.11 & 0.15 & 0.15 & 0.15 & 0.16 & 0.15 \\
\hline LOI & & & & 1.59 & 0.98 & 2.36 & 1.00 & 0.83 & 0.91 & 1.18 & 0.64 & 0.45 & 0.26 & 0.64 & 0.72 & 0.47 & 0.46 \\
\hline Total: & 100.00 & 99.97 & & 100.38 & 100.94 & 101.52 & 100.25 & 100.62 & 100.31 & 100.58 & 100.61 & 100.18 & 100.53 & 100.51 & 100.27 & 100.41 & 100.10 \\
\hline Mg\#: & & & & 59.2 & 46.4 & 56.7 & 59.4 & 55.6 & 48.6 & 53.5 & 52.3 & 52.9 & 49.0 & 46.2 & 44.2 & 42.9 & 46.9 \\
\hline \multicolumn{18}{|c|}{ Trace element (ppm): } \\
\hline Sc & 36.20 & 35.23 & 2.94 & 45.23 & 47.25 & 47.68 & 44.63 & 43.22 & 45.01 & 46.44 & 42.45 & 43.29 & 46.91 & 45.43 & 47.14 & 46.07 & 48.99 \\
\hline $\mathrm{V}$ & 270.0 & 262.3 & 17.4 & 280.4 & 413.0 & 345.1 & 304.6 & 335.7 & 382.8 & 389.6 & 345.4 & 336.1 & 429.1 & 418.4 & 420.6 & 449.2 & 456.8 \\
\hline $\mathrm{Ga}$ & 17.40 & 17.46 & 0.94 & 14.98 & 18.24 & 15.10 & 15.68 & 15.64 & 17.64 & 16.17 & 15.95 & 16.25 & 18.14 & 17.75 & 18.04 & 18.23 & 19.64 \\
\hline $\mathrm{Rb}$ & 20.10 & 20.65 & 0.25 & 0.31 & 0.54 & 1.50 & 0.18 & 0.94 & 0.83 & 1.65 & 0.53 & 0.13 & 0.19 & 0.59 & 0.37 & 0.39 & 0.23 \\
\hline $\mathrm{Sr}$ & 191.8 & 194.5 & 3.5 & 70.4 & 84.6 & 86.8 & 83.1 & 73.5 & 83.6 & 103.2 & 90.7 & 71.4 & 95.9 & 86.5 & 76.3 & 79.0 & 96.8 \\
\hline $\mathrm{Y}$ & 22.80 & 23.08 & 0.58 & 25.4 & 43.4 & 35.1 & 27.8 & 31.0 & 38.2 & 35.2 & 34.1 & 32.7 & 41.4 & 42.4 & 44.0 & 45.8 & 44.9 \\
\hline $\mathrm{Zr}$ & 92.00 & 98.13 & 2.58 & 53.3 & 101.1 & 82.3 & 59.2 & 69.9 & 89.1 & 84.0 & 75.7 & 76.4 & 93.9 & 99.2 & 113.9 & 117.8 & 96.5 \\
\hline Cs & 0.916 & 0.913 & 0.017 & 0.009 & 0.012 & 0.010 & 0.005 & 0.001 & 0.010 & 0.004 & 0.002 & 0.001 & 0.001 & 0.003 & 0.003 & 0.001 & 0.002 \\
\hline $\mathrm{Ba}$ & 171.0 & 174.7 & 2.81 & 10.31 & 18.81 & 29.52 & 6.26 & 15.19 & 12.57 & 35.72 & 10.03 & 7.97 & 9.24 & 15.73 & 11.78 & 11.39 & 12.29 \\
\hline La & 10.59 & 10.88 & 0.22 & 2.27 & 3.57 & 3.23 & 2.06 & 2.20 & 3.08 & 2.46 & 2.93 & 2.69 & 3.37 & 3.52 & 4.03 & 4.10 & 3.97 \\
\hline $\mathrm{Ce}$ & 23.08 & 23.60 & 0.31 & 6.46 & 10.64 & 9.29 & 6.22 & 7.08 & 9.11 & 7.74 & 9.25 & 8.18 & 10.37 & 10.69 & 11.70 & 12.14 & 11.61 \\
\hline $\mathrm{Pr}$ & 3.03 & 3.07 & 0.04 & 1.10 & 1.96 & 1.70 & 1.10 & 1.23 & 1.57 & 1.39 & 1.48 & 1.40 & 1.75 & 1.91 & 2.03 & 2.07 & 1.90 \\
\hline $\mathrm{Nd}$ & 12.95 & 13.25 & 0.39 & 5.78 & 10.17 & 8.67 & 6.19 & 7.01 & 8.83 & 7.69 & 8.36 & 7.75 & 9.34 & 10.26 & 11.01 & 11.30 & 10.68 \\
\hline $\mathrm{Sm}$ & 3.31 & 3.37 & 0.08 & 2.09 & 3.74 & 3.12 & 2.32 & 2.54 & 3.32 & 2.87 & 3.11 & 2.81 & 3.56 & 3.62 & 3.88 & 3.84 & 3.98 \\
\hline Eu & 1.09 & 1.13 & 0.04 & 0.77 & 1.33 & 1.06 & 0.82 & 0.95 & 1.17 & 1.05 & 1.14 & 0.99 & 1.30 & 1.30 & 1.39 & 1.39 & 1.36 \\
\hline $\mathrm{Gd}$ & 3.69 & 3.74 & 0.10 & 3.02 & 5.47 & 4.42 & 3.40 & 3.71 & 4.55 & 4.26 & 4.22 & 3.98 & 5.30 & 5.29 & 5.59 & 5.89 & 5.65 \\
\hline $\mathrm{Tb}$ & 0.62 & 0.62 & 0.02 & 0.59 & 1.08 & 0.87 & 0.65 & 0.74 & 0.91 & 0.84 & 0.85 & 0.82 & 1.03 & 1.05 & 1.06 & 1.11 & 1.04 \\
\hline Dy & 3.79 & 3.84 & 0.10 & 3.83 & 6.73 & 5.41 & 4.27 & 4.91 & 5.90 & 5.35 & 5.51 & 5.13 & 6.37 & 6.77 & 6.69 & 7.04 & 6.88 \\
\hline Ho & 0.80 & 0.82 & 0.03 & 0.83 & 1.51 & 1.22 & 0.97 & 1.05 & 1.30 & 1.18 & 1.20 & 1.14 & 1.44 & 1.50 & 1.53 & 1.61 & 1.59 \\
\hline $\mathrm{Er}$ & 2.26 & 2.29 & 0.07 & 2.63 & 4.52 & 3.70 & 2.86 & 3.19 & 4.07 & 3.61 & 3.60 & 3.49 & 4.17 & 4.43 & 4.44 & 4.61 & 4.59 \\
\hline $\mathrm{Yb}$ & 2.03 & 2.07 & 0.05 & 2.49 & 4.35 & 3.43 & 2.80 & 3.06 & 3.84 & 3.45 & 3.44 & 3.27 & 4.19 & 4.28 & 4.36 & 4.58 & 4.41 \\
\hline Lu & 0.30 & 0.31 & 0.01 & 0.39 & 0.68 & 0.55 & 0.43 & 0.48 & 0.61 & 0.55 & 0.55 & 0.52 & 0.65 & 0.67 & 0.69 & 0.71 & 0.66 \\
\hline $\mathrm{Hf}$ & 2.30 & 2.45 & 0.06 & 1.49 & 2.75 & 2.23 & 1.56 & 1.77 & 2.33 & 2.20 & 1.97 & 1.92 & 2.40 & 2.63 & 2.93 & 2.96 & 2.57 \\
\hline Th & 2.21 & 2.28 & 0.07 & 0.19 & 0.26 & 0.25 & 0.13 & 0.13 & 0.23 & 0.21 & 0.20 & 0.18 & 0.19 & 0.26 & 0.28 & 0.31 & 0.21 \\
\hline U & 0.50 & 0.50 & 0.01 & 0.06 & 0.07 & 0.07 & 0.04 & 0.04 & 0.07 & 0.06 & 0.07 & 0.05 & 0.06 & 0.08 & 0.08 & 0.09 & 0.07 \\
\hline
\end{tabular}

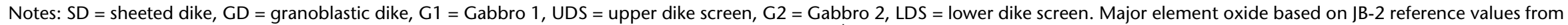
Imai et al. (1995), trace elements based on W-2 reference values from Eggins et al. (1997). $\mathrm{FeO}^{\text {total }}=$ total $\mathrm{Fe}$ as FeO. 
Table T2 (continued). (Continued on next page.)

\begin{tabular}{|c|c|c|c|c|c|c|c|c|c|c|c|c|c|}
\hline Core, section: & $214 R-2$ & $215 R-1$ & $215 R-2$ & 216R-1 & 219R-1 & $221 \mathrm{R}-1$ & $222 \mathrm{R}-1$ & $222 \mathrm{R}-2$ & $223 R-1$ & $223 R-1$ & $223 R-2$ & $227 \mathrm{R}-1$ & $227 \mathrm{R}-2$ \\
\hline Interval $(\mathrm{cm})$ : & $24-27$ & $20-23$ & $56-59$ & $138-142$ & $5-8$ & $61-64$ & $73-78$ & $60-63$ & $8-12$ & $88-91$ & 133-137 & $72-76$ & $50-59$ \\
\hline Depth (mbsf): & 1412.60 & 1415.92 & 1417.69 & 1419.19 & 1430.05 & 1440.21 & 1445.33 & 1446.70 & 1449.37 & 1450.18 & 1452.11 & 1469.21 & 1470.51 \\
\hline Lithology: & G1 & G1 & G1 & G1 & G1 & G1 & G1 & G1 & G1 & G1 & G1 & UDS & UDS \\
\hline Rock name & $\begin{array}{l}\text { Disseminated } \\
\text { oxide gabbro }\end{array}$ & $\begin{array}{c}\text { Disseminated } \\
\text { oxide olivine } \\
\text { gabbro }\end{array}$ & $\begin{array}{l}\text { Disseminated } \\
\text { oxide olivine } \\
\text { gabbro }\end{array}$ & $\begin{array}{l}\text { Olivine } \\
\text { gabbro }\end{array}$ & $\begin{array}{c}\text { Orthopyroxene- } \\
\text { bearing olivine } \\
\text { gabbro }\end{array}$ & $\begin{array}{l}\text { Orthopyroxene- } \\
\text { bearing olivine }\end{array}$ & $\begin{array}{c}\text { Orthopyroxene- } \\
\text { bearing olivine } \\
\text { gabbro }\end{array}$ & $\begin{array}{l}\text { Olivine } \\
\text { gabbro }\end{array}$ & $\begin{array}{l}\text { Orthopyroxene- } \\
\text { bearing olivine } \\
\text { gabbro }\end{array}$ & $\begin{array}{c}\text { Orthopyroxene- } \\
\text { bearing olivine } \\
\text { gabbro }\end{array}$ & $\begin{array}{c}\text { Olivine } \\
\text { gabbronorite }\end{array}$ & $\begin{array}{c}\text { Type } 8 \\
\text { metabasalt }\end{array}$ & $\begin{array}{c}\text { Type } 8 \\
\text { metabasalt }\end{array}$ \\
\hline \multicolumn{14}{|c|}{ Major element oxide (wt\%): } \\
\hline $\mathrm{SiO}_{2}$ & 49.63 & 49.90 & 48.20 & 49.74 & 50.17 & 51.16 & 47.74 & 49.02 & 47.76 & 48.46 & 50.02 & 50.40 & 50.40 \\
\hline $\mathrm{TiO}_{2}$ & 1.17 & 1.36 & 0.65 & 0.92 & 0.83 & 1.56 & 0.65 & 0.51 & 0.49 & 0.69 & 0.82 & 1.55 & 1.56 \\
\hline $\mathrm{Al}_{2} \mathrm{O}_{3}$ & 15.84 & 16.10 & 14.27 & 15.76 & 15.35 & 14.99 & 14.15 & 15.89 & 14.7 & 15.7 & 14.63 & 13.57 & 13.74 \\
\hline $\mathrm{FeO}^{\text {total }}$ & 9.71 & 9.83 & 9.03 & 8.06 & 8.54 & 10.13 & 8.99 & 7.81 & 8.59 & 8.09 & 8.46 & 13.06 & 12.32 \\
\hline $\mathrm{MnO}$ & 0.14 & 0.14 & 0.13 & 0.13 & 0.15 & 0.17 & 0.13 & 0.12 & 0.13 & 0.12 & 0.15 & 0.22 & 0.17 \\
\hline $\mathrm{MgO}$ & 7.54 & 7.05 & 12.85 & 8.50 & 8.57 & 7.20 & 12.72 & 9.96 & 12.07 & 11.11 & 10.20 & 7.58 & 7.42 \\
\hline $\mathrm{CaO}$ & 12.61 & 11.89 & 12.07 & 13.50 & 12.99 & 11.00 & 12.01 & 13.20 & 12.64 & 12.36 & 13.14 & 11.23 & 11.49 \\
\hline $\mathrm{Na}_{2} \mathrm{O}$ & 2.55 & 2.92 & 2.16 & 2.42 & 2.30 & 3.12 & 2.07 & 2.30 & 1.90 & 2.27 & 2.23 & 2.47 & 2.66 \\
\hline $\mathrm{K}_{2} \mathrm{O}$ & 0.07 & 0.09 & 0.08 & 0.09 & 0.10 & 0.09 & 0.08 & 0.08 & 0.10 & 0.09 & 0.08 & 0.03 & 0.04 \\
\hline $\mathrm{P}_{2} \mathrm{O}_{5}$ & 0.07 & 0.10 & 0.04 & 0.07 & 0.07 & 0.17 & 0.04 & 0.04 & 0.03 & 0.04 & 0.05 & 0.09 & 0.10 \\
\hline LOI & 0.60 & 0.63 & 0.64 & 0.67 & 0.71 & 0.78 & 1.29 & 0.73 & 1.48 & 1.11 & 0.52 & 0.37 & 0.46 \\
\hline Total: & 99.93 & 100.00 & 100.12 & 99.85 & 99.77 & 100.36 & 99.86 & 99.66 & 99.89 & 100.04 & 100.30 & 100.57 & 100.37 \\
\hline Mg\#: & 58.1 & 56.1 & 71.7 & 65.3 & 64.1 & 55.9 & 71.5 & 69.5 & 71.5 & 71.0 & 68.2 & 50.8 & 51.8 \\
\hline \multicolumn{14}{|c|}{ Trace element (ppm): } \\
\hline $\mathrm{Sc}$ & 41.77 & 40.09 & 42.59 & 40.66 & 44.46 & 41.94 & 38.40 & 38.21 & 38.19 & 32.91 & 46.54 & 46.91 & 46.23 \\
\hline V & 272.8 & 287.4 & 310.5 & 240.9 & 232.9 & 429.3 & 213.7 & 179.2 & 171.2 & 170.1 & 254.3 & 461.1 & 412.7 \\
\hline Ga & 15.38 & 16.49 & 16.04 & 13.61 & 14.78 & 19.49 & 12.74 & 13.69 & 12.92 & 13.43 & 14.74 & 17.85 & 17.62 \\
\hline $\mathrm{Rb}$ & 0.45 & 0.44 & 0.46 & 0.45 & 0.71 & 0.34 & 0.31 & 0.42 & 0.71 & 0.32 & 0.59 & 0.13 & 0.22 \\
\hline $\mathrm{Sr}$ & 92.0 & 99.2 & 98.2 & 84.6 & 90.6 & 97.9 & 78.0 & 88.6 & 76.1 & 85.6 & 88.6 & 82.4 & 79.8 \\
\hline$Y$ & 26.0 & 26.4 & 27.4 & 21.0 & 22.9 & 43.3 & 16.4 & 15.8 & 13.3 & 16.1 & 23.2 & 33.6 & 35.1 \\
\hline $\mathrm{Zr}$ & 51.4 & 65.8 & 69.0 & 44.0 & 55.2 & 148.5 & 33.2 & 28.5 & 23.3 & 38.1 & 44.2 & 80.0 & 80.7 \\
\hline Cs & 0.004 & 0.004 & 0.004 & 0.003 & 0.007 & 0.003 & 0.006 & 0.002 & 0.007 & 0.000 & 0.003 & 0.005 & 0.004 \\
\hline $\mathrm{Ba}$ & 9.62 & 9.74 & 10.83 & 21.94 & 17.47 & 9.52 & 8.89 & 8.04 & 9.07 & 6.57 & 9.75 & 7.02 & 6.31 \\
\hline La & 2.33 & 2.60 & 2.93 & 2.26 & 1.83 & 3.65 & 2.01 & 1.65 & 1.41 & 1.88 & 1.82 & 2.84 & 2.87 \\
\hline $\mathrm{Ce}$ & 7.35 & 7.50 & 7.94 & 7.08 & 5.54 & 11.21 & 5.80 & 4.86 & 3.59 & 5.04 & 5.48 & 8.41 & 9.01 \\
\hline $\mathrm{Pr}$ & 1.27 & 1.22 & 1.37 & 1.28 & 0.92 & 2.03 & 0.91 & 0.81 & 0.55 & 0.78 & 0.95 & 1.44 & 1.53 \\
\hline $\mathrm{Nd}$ & 6.94 & 6.53 & 7.14 & 6.57 & 5.31 & 10.68 & 4.61 & 4.13 & 3.14 & 4.07 & 5.27 & 8.01 & 8.21 \\
\hline $\mathrm{Sm}$ & 2.44 & 2.39 & 2.51 & 2.22 & 1.93 & 3.97 & 1.53 & 1.51 & 1.16 & 1.40 & 1.92 & 2.94 & 3.03 \\
\hline $\mathrm{Eu}$ & 0.97 & 1.09 & 0.99 & 0.90 & 0.83 & 1.14 & 0.75 & 0.70 & 0.60 & 0.72 & 0.80 & 1.05 & 1.15 \\
\hline $\mathrm{Gd}$ & 3.34 & 3.29 & 3.50 & 3.01 & 2.73 & 5.65 & 2.16 & 1.97 & 1.67 & 1.99 & 2.82 & 4.17 & 4.38 \\
\hline $\mathrm{Tb}$ & 0.64 & 0.64 & 0.68 & 0.52 & 0.54 & 1.05 & 0.39 & 0.37 & 0.32 & 0.37 & 0.53 & 0.79 & 0.85 \\
\hline Dy & 4.12 & 4.11 & 4.34 & 3.36 & 3.59 & 6.89 & 2.55 & 2.33 & 2.02 & 2.45 & 3.53 & 5.37 & 5.71 \\
\hline Ho & 0.91 & 0.96 & 0.96 & 0.73 & 0.77 & 1.54 & 0.57 & 0.55 & 0.46 & 0.57 & 0.81 & 1.19 & 1.23 \\
\hline $\mathrm{Er}$ & 2.61 & 2.72 & 2.69 & 2.14 & 2.31 & 4.44 & 1.65 & 1.60 & 1.36 & 1.60 & 2.33 & 3.48 & 3.63 \\
\hline $\mathrm{Yb}$ & 2.50 & 2.57 & 2.67 & 1.99 & 2.26 & 4.27 & 1.56 & 1.47 & 1.30 & 1.54 & 2.22 & 3.33 & 3.41 \\
\hline Lu & 0.39 & 0.39 & 0.40 & 0.29 & 0.33 & 0.63 & 0.24 & 0.23 & 0.20 & 0.23 & 0.33 & 0.51 & 0.53 \\
\hline $\mathrm{Hf}$ & 1.39 & 1.75 & 1.80 & 1.17 & 1.46 & 3.69 & 0.83 & 0.75 & 0.63 & 0.95 & 1.20 & 2.01 & 2.05 \\
\hline Th & 0.13 & 0.13 & 0.15 & 0.11 & 0.12 & 0.36 & 0.08 & 0.07 & 0.05 & 0.08 & 0.09 & 0.21 & 0.19 \\
\hline U & 0.04 & 0.05 & 0.05 & 0.03 & 0.04 & 0.11 & 0.03 & 0.03 & 0.02 & 0.05 & 0.03 & 0.07 & 0.06 \\
\hline
\end{tabular}




\begin{tabular}{|c|c|c|c|c|c|}
\hline Core, section: & $230 \mathrm{R}-2$ & $231 \mathrm{R}-3$ & $231 \mathrm{R}-3$ & 234R-1 & 234R-1 \\
\hline Interval $(\mathrm{cm})$ : & 104-109 & $59-63$ & $123-127$ & $1-2$ & $7-9$ \\
\hline Depth (mbsf): & 1485.54 & 1491.15 & 1491.79 & 1502.50 & 1502.57 \\
\hline Lithology: & G2 & G2 & $\mathrm{G} 2$ & LDS & LDS \\
\hline Rock name & Gabbronorite & $\begin{array}{l}\text { Disseminated } \\
\text { oxide olivine- } \\
\text { bearing } \\
\text { gabbronorite }\end{array}$ & $\begin{array}{c}\text { Disseminated } \\
\text { oxide } \\
\text { gabbronorite }\end{array}$ & $\begin{array}{c}\text { Type } 8 \\
\text { metabasalt }\end{array}$ & $\begin{array}{c}\text { Type } 8 \\
\text { metabasal }\end{array}$ \\
\hline \multicolumn{6}{|c|}{ Major element oxide (wt\%): } \\
\hline $\mathrm{SiO}_{2}$ & 50.48 & 49.78 & 50.78 & 49.05 & 49.58 \\
\hline $\mathrm{TiO}_{2}$ & 1.16 & 1.15 & 0.96 & 1.38 & 1.37 \\
\hline $\mathrm{Al}_{2} \mathrm{O}_{3}$ & 14.38 & 14.65 & 14.65 & 13.91 & 13.86 \\
\hline $\mathrm{FeO}^{\text {total }}$ & 9.49 & 9.96 & 9.09 & 12.16 & 12.1 \\
\hline $\mathrm{MnO}$ & 0.16 & 0.17 & 0.16 & 0.16 & 0.17 \\
\hline $\mathrm{MgO}$ & 8.25 & 8.95 & 8.63 & 8.28 & 8.27 \\
\hline $\mathrm{CaO}$ & 12.7 & 12.55 & 12.78 & 12.09 & 12.04 \\
\hline $\mathrm{Na}_{2} \mathrm{O}$ & 2.56 & 2.39 & 2.41 & 2.41 & 2.44 \\
\hline $\mathrm{K}_{2} \mathrm{O}$ & 0.05 & 0.06 & 0.05 & 0.02 & 0.02 \\
\hline $\mathrm{P}_{2} \mathrm{O}_{5}$ & 0.08 & 0.04 & 0.03 & 0.02 & 0.03 \\
\hline LOI & 0.88 & 0.61 & 0.57 & 0.94 & 0.42 \\
\hline Total: & $: \quad 100.18$ & 100.29 & 100.10 & 100.43 & 100.29 \\
\hline Mg\#: & 60.8 & 61.6 & 62.9 & 54.8 & 54.9 \\
\hline \multicolumn{6}{|c|}{ Trace element (ppm): } \\
\hline Sc & 46.21 & 45.51 & 48.85 & 47.13 & 45.93 \\
\hline $\mathrm{V}$ & 283.6 & 340.0 & 331.3 & 394.1 & 387.8 \\
\hline $\mathrm{Ga}$ & 15.48 & 15.17 & 15.71 & 16.11 & 16.51 \\
\hline $\mathrm{Rb}$ & 0.28 & 0.24 & 0.21 & 0.10 & 1.83 \\
\hline $\mathrm{Sr}$ & 92.4 & 96.0 & 97.2 & 88.3 & 92.5 \\
\hline $\mathrm{Y}$ & 31.6 & 21.5 & 25.4 & 18.5 & 19.8 \\
\hline $\mathrm{Zr}$ & 62.3 & 34.1 & 32.0 & 15.5 & 19.7 \\
\hline Cs & 0.003 & 0.002 & - & 0.003 & 0.008 \\
\hline $\mathrm{Ba}$ & 9.03 & 7.33 & 7.20 & 5.16 & 5.60 \\
\hline La & 2.48 & 1.37 & 1.45 & 0.74 & 0.72 \\
\hline $\mathrm{Ce}$ & 7.28 & 4.11 & 4.83 & 2.40 & 2.41 \\
\hline $\mathrm{Pr}$ & 1.28 & 0.73 & 0.89 & 0.46 & 0.50 \\
\hline $\mathrm{Nd}$ & 7.42 & 3.97 & 4.87 & 2.97 & 3.03 \\
\hline $\mathrm{Sm}$ & 2.92 & 1.73 & 2.01 & 1.36 & 1.49 \\
\hline $\mathrm{Eu}$ & 1.00 & 0.85 & 0.86 & 0.70 & 0.73 \\
\hline $\mathrm{Gd}$ & 4.03 & 2.43 & 3.14 & 2.14 & 2.32 \\
\hline $\mathrm{Tb}$ & 0.78 & 0.48 & 0.57 & 0.44 & 0.45 \\
\hline Dy & 5.02 & 3.28 & 3.82 & 2.90 & 3.12 \\
\hline Ho & 1.12 & 0.76 & 0.88 & 0.64 & 0.72 \\
\hline $\mathrm{Er}$ & 3.23 & 2.23 & 2.57 & 1.92 & 2.04 \\
\hline $\mathrm{Yb}$ & 3.04 & 2.09 & 2.47 & 1.77 & 1.94 \\
\hline Lu & 0.46 & 0.31 & 0.36 & 0.28 & 0.30 \\
\hline $\mathrm{Hf}$ & 1.65 & 0.95 & 0.89 & 0.56 & 0.68 \\
\hline Th & 0.15 & 0.07 & 0.10 & 0.02 & 0.02 \\
\hline U & 0.04 & 0.02 & 0.03 & 0.01 & 0.01 \\
\hline
\end{tabular}


Table T3. Average values of mineral compositions. (See table notes.) (Continued on next eight pages.)

\begin{tabular}{|c|c|c|c|c|c|c|c|c|c|c|c|c|c|c|c|c|c|c|c|c|c|c|}
\hline \multirow{2}{*}{$\begin{array}{l}\text { Core, section, } \\
\text { interval }(\mathrm{cm})\end{array}$} & \multirow{2}{*}{$\begin{array}{l}\text { Depth } \\
\text { (mbsf) }\end{array}$} & \multirow[b]{2}{*}{ Rock name } & \multirow[b]{2}{*}{ Phase } & \multirow[b]{2}{*}{ Replicates } & \multirow{2}{*}{$\begin{array}{c}\text { Mode of } \\
\text { occurrence }\end{array}$} & \multirow[b]{2}{*}{$\mathrm{AP}$} & \multicolumn{16}{|c|}{ Major element oxide (wt\%) } \\
\hline & & & & & & & $\mathrm{SiO}_{2}$ & $\mathrm{TiO}_{2}$ & $\mathrm{Al}_{2} \mathrm{O}_{3}$ & $\mathrm{Cr}_{2} \mathrm{O}_{3}$ & $\mathrm{FeO}$ & $\mathrm{MnO}$ & $\mathrm{MgO}$ & $\mathrm{CaO}$ & $\mathrm{Na}_{2} \mathrm{O}$ & $\mathrm{K}_{2} \mathrm{O}$ & $\mathrm{NiO}$ & $\mathrm{BaO}$ & Total & An $\%$ & Mg\# & Fo \\
\hline \multirow{14}{*}{$\begin{array}{l}\text { 312-1256D- } \\
172 \mathrm{R}-1,12-14\end{array}$} & & & & & & & & & & & & & & & & & & & & & & \\
\hline & 1255.20 & $\begin{array}{l}\text { Type } 3 \\
\text { metabasalt }\end{array}$ & $\mathrm{PI}$ & $N=3$ & Phenocryst & Core & 48.3 & - & 31.7 & - & 0.6 & - & 0.3 & 16.2 & 2.48 & - & - & - & 99.6 & 78.4 & & \\
\hline & & & & SD & & & 1.2 & & 0.9 & & 0.1 & - & 0.0 & 1.0 & 0.59 & - & - & - & 0.2 & 5.1 & & \\
\hline & & & $\mathrm{PI}$ & $\begin{array}{l}N=1 \\
S D\end{array}$ & Phenocryst & $\operatorname{Rim}$ & 51.0 & - & 29.6 & - & 0.7 & - & 0.3 & 14.1 & 3.62 & - & - & - & 99.4 & 68.2 & & \\
\hline & & & $\mathrm{Pl}$ & $N=3$ & Lath & Core & 50.6 & - & 30.7 & - & 0.7 & - & 0.3 & 14.9 & 3.13 & - & - & - & 100.4 & 72.5 & & \\
\hline & & & & SD & & & 1.4 & & 1.0 & & 0.1 & & 0.0 & 1.1 & 0.65 & & & & 0.2 & 5.7 & & \\
\hline & & & $\mathrm{Pl}$ & $N=3$ & Lath & $\operatorname{Rim}$ & 50.7 & - & 30.6 & - & 0.6 & - & 0.3 & 14.8 & 3.23 & - & - & - & 100.3 & 71.8 & & \\
\hline & & & & $\mathrm{SD}$ & & & 1.5 & & 0.8 & & 0.1 & & 0.0 & 1.1 & 0.71 & & & & 0.4 & 6.0 & & \\
\hline & & & Cpx & $N=4$ & Phenocryst & Core & 52.5 & 0.20 & 2.5 & 0.75 & 5.5 & 0.15 & 19.0 & 19.4 & 0.21 & - & - & - & 100.3 & & 86.0 & \\
\hline & & & & SD & & & 0.2 & 0.04 & 0.5 & 0.14 & 0.1 & 0.01 & 0.5 & 0.4 & 0.01 & & & & 0.1 & & 0.2 & \\
\hline & & & $\mathrm{Cpx}$ & $N=4$ & Phenocryst & $\operatorname{Rim}$ & 52.2 & 0.37 & 2.9 & 0.32 & 6.6 & 0.18 & 18.4 & 19.1 & 0.20 & - & - & - & 100.3 & & 83.2 & \\
\hline & & & & SD & & & 0.4 & 0.09 & 0.6 & 0.03 & 0.7 & 0.04 & 0.3 & 0.9 & 0.01 & & & & 0.4 & & 1.7 & \\
\hline & & & $\mathrm{Cpx}$ & $N=6$ & Microlite & Core & 51.9 & 0.40 & 3.3 & 0.29 & 6.9 & 0.20 & 18.4 & 18.7 & 0.22 & - & - & - & 100.4 & & 82.5 & \\
\hline & & & & SD & & & 0.5 & 0.12 & 0.7 & 0.18 & 0.8 & 0.03 & 0.9 & 0.8 & 0.02 & & & & 0.1 & & 2.2 & \\
\hline \multirow[t]{6}{*}{$174 \mathrm{R}-1,32-34$} & 1265.71 & $\begin{array}{l}\text { Type } 3 \\
\text { metabasalt }\end{array}$ & Cpx & $N=3$ & Phenocryst & Core & 51.5 & 0.29 & 3.5 & 1.02 & 5.4 & 0.12 & 18.3 & 19.5 & 0.19 & - & - & - & 99.9 & & 85.7 & \\
\hline & & & & SD & & & 0.4 & 0.03 & 0.8 & 0.06 & 0.2 & 0.03 & 0.2 & 0.8 & 0.01 & & & & 0.6 & & 0.4 & \\
\hline & & & Cpx & $N=3$ & Phenocryst & $\operatorname{Rim}$ & 51.9 & 0.44 & 2.8 & 0.34 & 7.1 & 0.12 & 18.1 & 18.5 & 0.18 & - & - & - & 99.5 & & 82.0 & \\
\hline & & & & SD & & & 0.1 & 0.03 & 0.1 & 0.10 & 0.1 & & 0.2 & 0.6 & 0.02 & & & & 0.6 & & 0.2 & \\
\hline & & & $\mathrm{Cpx}$ & $N=2$ & Microlite & Core & 51.2 & 0.48 & 3.5 & 0.22 & 7.5 & 0.16 & 17.8 & 18.7 & 0.19 & - & - & - & 99.9 & & 80.8 & \\
\hline & & & & SD & & & 0.2 & 0.02 & 0.3 & 0.08 & 0.9 & & 0.1 & 1.1 & 0.00 & & & & 0.6 & & 1.7 & \\
\hline \multirow[t]{5}{*}{$176 \mathrm{R}-1,21-24$} & 1276.29 & $\begin{array}{l}\text { Type } 3 \\
\text { metabasalt }\end{array}$ & $\mathrm{PI}$ & $N=1$ & Lath & Core & 51.8 & - & 29.3 & - & 0.8 & - & 0.2 & 12.8 & 4.18 & - & - & - & 99.1 & 62.8 & & \\
\hline & & & & SD & & & & & & & & & & & & & & & & & & \\
\hline & & & $\mathrm{Pl}$ & $\begin{array}{l}N=1 \\
S D\end{array}$ & Lath & $\operatorname{Rim}$ & 49.2 & - & 31.0 & - & 0.6 & - & 0.3 & 15.3 & 2.84 & - & - & - & 99.3 & 74.9 & & \\
\hline & & & $\mathrm{Cpx}$ & $N=6$ & Microlite & Core & 51.0 & 0.58 & 3.8 & 0.18 & 8.5 & 0.20 & 16.4 & 18.9 & 0.38 & - & - & - & 100.1 & & 77.3 & \\
\hline & & & & SD & & & 0.3 & 0.06 & 1.6 & 0.09 & 1.4 & 0.04 & 1.6 & 1.1 & 0.37 & & & & 0.6 & & 4.8 & \\
\hline \multirow[t]{6}{*}{$178 \mathrm{R}-1,0-3$} & 1285.70 & $\begin{array}{l}\text { Type } 3 \\
\text { metabasalt }\end{array}$ & $\mathrm{PI}$ & $N=3$ & Lath & Core & 50.9 & - & 30.0 & - & 0.6 & - & 0.3 & 14.4 & 3.43 & - & - & - & 99.7 & 69.8 & & \\
\hline & & & & SD & & & 0.3 & & 0.2 & & 0.0 & & 0.0 & 0.1 & 0.11 & & & & 0.1 & 0.9 & & \\
\hline & & & $\mathrm{Pl}$ & $N=2$ & Lath & $\operatorname{Rim}$ & 52.4 & - & 29.0 & - & 0.7 & - & 0.2 & 13.0 & 4.20 & - & - & - & 99.6 & 63.1 & & \\
\hline & & & & SD & & & 1.0 & & 0.7 & & 0.2 & & 0.1 & 0.8 & 0.60 & & & & 0.1 & 4.9 & & \\
\hline & & & Cpx & $N=2$ & Microlite & Core & 51.7 & 0.56 & 3.5 & - & 8.8 & 0.21 & 16.5 & 18.7 & 0.38 & - & - & - & 100.0 & & 76.9 & \\
\hline & & & & SD & & & 0.0 & 0.05 & 0.2 & & 0.2 & 0.00 & 0.2 & 0.0 & 0.00 & & & & 0.1 & & 0.6 & \\
\hline \multirow[t]{3}{*}{$193 R-1,5-8$} & 1353.10 & $\begin{array}{l}\text { Type } 6 \\
\text { metabasalt }\end{array}$ & $\mathrm{Pl}$ & $N=3$ & Microlite & Core & 53.2 & - & 28.2 & - & 0.9 & - & 0.1 & 11.7 & 4.89 & - & - & - & 99.1 & 57.0 & & \\
\hline & & & & SD & & & 0.0 & & 0.2 & & 0.1 & & 0.0 & 0.2 & 0.05 & & & & 0.1 & 0.4 & & \\
\hline & & & Cpx & $N=1$ & Phenocryst & Core & 51.7 & 0.54 & 2.7 & - & 8.9 & 0.21 & 16.5 & 19.0 & 0.30 & - & - & - & 99.9 & & 76.6 & \\
\hline \multirow[t]{6}{*}{ 194R-1, 29-33 } & 1358.19 & $\begin{array}{l}\text { Type } 7 \\
\text { metabasalt }\end{array}$ & $\mathrm{Pl}$ & $N=4$ & Lath & Core & 51.9 & 0.07 & 29.4 & - & 0.8 & - & 0.1 & 13.3 & 4.03 & - & - & - & 99.7 & 64.6 & & \\
\hline & & & & SD & & & 2.5 & & 1.8 & & 0.1 & & 0.0 & 2.1 & 1.12 & & & & 0.4 & 10.0 & & \\
\hline & & & $\mathrm{Pl}$ & $N=2$ & Lath & $\operatorname{Rim}$ & 51.9 & - & 29.5 & - & 0.7 & - & 0.1 & 13.3 & 4.10 & - & - & - & 99.8 & 64.3 & & \\
\hline & & & & SD & & & 3.5 & & 1.9 & & 0.0 & & 0.0 & 2.5 & 1.53 & & & & 0.7 & 12.9 & & \\
\hline & & & Cpx & $N=9$ & Microlite & Core & 51.3 & 0.47 & 1.2 & - & 11.9 & 0.29 & 14.4 & 19.8 & 0.25 & - & - & - & 99.7 & & 68.4 & \\
\hline & & & & SD & & & 0.3 & 0.04 & 0.1 & & 0.3 & 0.04 & 0.1 & 0.6 & 0.01 & & & & 0.4 & & 0.5 & \\
\hline
\end{tabular}


Table T3 (continued). (Continued on next page.)

\begin{tabular}{|c|c|c|c|c|c|c|c|c|c|c|c|c|c|c|c|c|c|c|c|c|c|c|}
\hline \multirow{2}{*}{$\begin{array}{l}\text { Core, section, } \\
\text { interval (cm) }\end{array}$} & \multirow{2}{*}{$\begin{array}{l}\text { Depth } \\
\text { (mbsf) }\end{array}$} & \multirow[b]{2}{*}{ Rock name } & \multirow[b]{2}{*}{ Phase } & \multirow[b]{2}{*}{ Replicates } & \multirow{2}{*}{$\begin{array}{l}\text { Mode of } \\
\text { occurrence }\end{array}$} & \multirow[b]{2}{*}{ AP } & \multicolumn{16}{|c|}{ Major element oxide (wt\%) } \\
\hline & & & & & & & $\mathrm{SiO}_{2}$ & $\mathrm{TiO}_{2}$ & $\mathrm{Al}_{2} \mathrm{O}_{3}$ & $\mathrm{Cr}_{2} \mathrm{O}_{3}$ & $\mathrm{FeO}$ & $\mathrm{MnO}$ & $\mathrm{MgO}$ & $\mathrm{CaO}$ & $\mathrm{Na}_{2} \mathrm{O}$ & $\mathrm{K}_{2} \mathrm{O}$ & $\mathrm{NiO}$ & $\mathrm{BaO}$ & Total & An\% & Mg\# & Fo \\
\hline \multirow[t]{5}{*}{ 196R-1, 43-46 } & 1364.13 & $\begin{array}{l}\text { Type } 7 \\
\text { metabasalt }\end{array}$ & $\mathrm{PI}$ & $N=4$ & Microlite & Core & 54.6 & - & 27.5 & - & 0.8 & - & 0.0 & 11.0 & 5.35 & 0.08 & - & - & 99.5 & 53.3 & & \\
\hline & & & & SD & & & 1.8 & & 1.3 & & 0.1 & & 0.0 & 1.1 & 0.66 & & & & 0.2 & 5.5 & & \\
\hline & & & $\mathrm{PI}$ & $N=4$ & Lath & Core & 52.3 & 0.09 & 29.1 & - & 0.8 & - & 0.1 & 12.4 & 4.43 & 0.07 & 0.17 & - & 99.3 & 60.8 & & \\
\hline & & & & SD & & & 2.8 & & 1.8 & & 0.0 & & 0.0 & 2.5 & 1.43 & & & & 0.2 & 12.6 & & \\
\hline & & & Cpx & $\begin{array}{l}N=1 \\
S D\end{array}$ & Secondary-type & Core & 53.2 & - & 0.4 & - & 8.3 & 0.32 & 15.2 & 21.3 & 0.20 & - & - & - & 99.1 & & 76.5 & \\
\hline \multirow[t]{8}{*}{ 203R-1, 6-10 } & 1374.89 & $\begin{array}{l}\text { Type } 7 \\
\text { metabasalt }\end{array}$ & $\mathrm{PI}$ & $N=22$ & Microlite & Core & 54.8 & - & 27.3 & - & 0.8 & - & 0.1 & 10.5 & 5.53 & - & - & - & 99.3 & 51.2 & & \\
\hline & & & & SD & & & 1.4 & & 0.4 & & 0.1 & & 0.0 & 0.4 & 0.19 & & & & 0.5 & 1.9 & & \\
\hline & & & $\mathrm{Cpx}$ & $N=3$ & Phenocryst & Core & 52.5 & 0.33 & 1.4 & 0.23 & 7.1 & 0.24 & 16.0 & 21.8 & 0.29 & - & - & - & 99.9 & & 80.0 & \\
\hline & & & & SD & & & 0.7 & 0.23 & 0.7 & & 1.4 & 0.02 & 0.9 & 1.2 & 0.04 & & & & 0.5 & & 3.7 & \\
\hline & & & $\mathrm{Cpx}$ & $N=3$ & Phenocryst & $\operatorname{Rim}$ & 52.3 & 0.38 & 1.1 & - & 8.0 & 0.24 & 15.7 & 21.9 & 0.30 & - & - & - & 100.0 & & 77.8 & \\
\hline & & & & SD & & & 0.2 & 0.20 & 0.5 & & 0.9 & 0.04 & 0.3 & 1.4 & 0.05 & & & & 0.2 & & 2.3 & \\
\hline & & & Cpx & $N=9$ & Microgranular & Core & 51.1 & 0.61 & 1.5 & - & 8.7 & 0.26 & 15.3 & 21.0 & 0.32 & - & - & - & 98.9 & & 75.8 & \\
\hline & & & & SD & & & 0.5 & 0.05 & 0.1 & & 0.4 & 0.03 & 0.3 & 0.5 & 0.02 & & & & 0.6 & & 0.9 & \\
\hline \multirow[t]{10}{*}{ 204R-1, 0-4 } & 1377.30 & $\begin{array}{l}\text { Type } 7 \\
\text { metabasalt }\end{array}$ & $\mathrm{PI}$ & $N=9$ & Subhedral & Core & 53.3 & 0.07 & 28.8 & - & 0.8 & - & 0.0 & 12.1 & 4.71 & - & - & - & 99.9 & 58.7 & & \\
\hline & & & & SD & & & 1.7 & & 1.1 & & 0.1 & & 0.0 & 1.5 & 0.78 & & & & 0.3 & 6.8 & & \\
\hline & & & $\mathrm{PI}$ & $N=5$ & Subhedral & $\operatorname{Rim}$ & 53.6 & - & 28.7 & - & 0.9 & - & 0.1 & 12.2 & 4.75 & - & - & - & 100.2 & 58.6 & & \\
\hline & & & & SD & & & 0.7 & & 0.3 & & 0.1 & & 0.0 & 0.5 & 0.19 & & & & 0.3 & 1.9 & & \\
\hline & & & $\mathrm{Cpx}$ & $N=4$ & Phenocryst & Core & 51.6 & 0.47 & 1.3 & - & 12.5 & 0.33 & 14.2 & 19.4 & 0.24 & - & - & - & 100.2 & & 67.0 & \\
\hline & & & & SD & & & 0.6 & 0.03 & 0.1 & & 0.4 & 0.02 & 0.1 & 0.6 & 0.02 & & & & 0.9 & & 0.5 & \\
\hline & & & Cpx & $N=4$ & Microgranular & Core & 51.4 & 0.61 & 2.1 & - & 12.7 & 0.34 & 13.9 & 19.1 & 0.40 & - & - & - & 100.8 & & 66.1 & \\
\hline & & & & SD & & & 0.4 & 0.14 & 1.7 & & 0.7 & 0.06 & 0.9 & 0.4 & 0.31 & & & & 0.1 & & 0.3 & \\
\hline & & & Opx & $N=8$ & Microgranular & & 52.3 & 0.33 & 0.7 & - & 23.4 & 0.53 & 21.7 & 1.9 & - & - & - & - & 100.9 & & 62.3 & \\
\hline & & & & SD & & & 0.5 & 0.03 & 0.0 & & 0.9 & 0.03 & 0.6 & 0.2 & 0.01 & & & & 0.6 & & 1.5 & \\
\hline \multirow[t]{10}{*}{ 214R-1, 19-21 } & 1411.10 & Gabbro 1 & $\mathrm{PI}$ & $N=4$ & Subhedral & Core & 51.9 & - & 29.7 & - & 0.7 & - & 0.1 & 13.3 & 4.15 & - & - & - & 99.8 & 63.9 & & \\
\hline & & & & SD & & & 1.5 & & 0.9 & & 0.1 & & 0.0 & 1.2 & 0.62 & & & & 0.4 & 5.5 & & \\
\hline & & & $\mathrm{PI}$ & $N=2$ & Subhedral & $\operatorname{Rim}$ & 52.3 & - & 29.5 & - & 0.7 & - & 0.1 & 12.8 & 4.39 & - & - & - & 99.7 & 61.9 & & \\
\hline & & & & SD & & & 3.1 & & 1.6 & & 0.2 & & 0.0 & 2.1 & 1.28 & & & & 0.9 & 10.7 & & \\
\hline & & & $\mathrm{Cpx}$ & $N=9$ & Igneous-type & Core & 51.0 & 0.46 & 3.4 & 0.73 & 7.2 & 0.12 & 16.8 & 20.2 & 0.24 & - & - & - & 100.1 & & 80.7 & \\
\hline & & & & SD & $\begin{array}{l}\text { Subophitic } \\
\text { domain }\end{array}$ & & 1.0 & 0.03 & 0.8 & 0.26 & 0.7 & 0.03 & 0.7 & 0.6 & 0.05 & & & & 0.6 & & 2.1 & \\
\hline & & & $C p x$ & $N=3$ & Igneous-type & Core & 52.1 & 0.66 & 1.8 & 0.26 & 10.7 & 0.21 & 15.3 & 20.0 & 0.25 & - & - & - & 101.2 & & 71.8 & \\
\hline & & & & SD & $\begin{array}{l}\text { Coarse-grained } \\
\text { domain }\end{array}$ & & 0.2 & 0.06 & 0.1 & & 0.3 & 0.02 & 0.5 & 0.5 & 0.02 & & & & 0.7 & & 1.2 & \\
\hline & & & Opx & $N=14$ & Anhedral & & 52.9 & 0.40 & 0.9 & - & 21.4 & 0.40 & 23.0 & 2.5 & - & - & - & - & 101.4 & & 64.8 & \\
\hline & & & & SD & & & 0.3 & 0.03 & 0.1 & & 2.1 & 0.06 & 1.2 & 0.9 & & & & & 0.8 & & 3.7 & \\
\hline \multirow[t]{6}{*}{$214 R-2,24-57$} & 1412.60 & Gabbro 1 & $\mathrm{PI}$ & $N=3$ & Subhedral & Core & 49.1 & - & 31.3 & - & 0.4 & - & 0.1 & 15.2 & 3.06 & - & - & - & 99.3 & 73.4 & & \\
\hline & & & & SD & & & 1.4 & & 0.8 & & 0.1 & & 0.0 & 1.1 & 0.64 & & & & 0.2 & 5.6 & & \\
\hline & & & $\mathrm{Cpx}$ & $N=6$ & Igneous-type & Core & 51.7 & 0.62 & 2.5 & 0.24 & 7.2 & 0.12 & 17.5 & 19.8 & 0.28 & - & - & - & 100.2 & & 81.3 & \\
\hline & & & & SD & $\begin{array}{l}\text { Subophitic } \\
\text { domain }\end{array}$ & & 0.6 & 0.05 & 0.5 & 0.06 & 0.5 & 0.02 & 0.5 & 0.7 & 0.06 & & & & 0.5 & & 1.5 & \\
\hline & & & $\mathrm{Cpx}$ & $N=3$ & Igneous-type & Core & 50.7 & 0.60 & 2.7 & 0.28 & 9.0 & 0.15 & 16.3 & 19.6 & 0.24 & - & - & - & 99.6 & & 76.3 & \\
\hline & & & & SD & $\begin{array}{l}\text { Coarse-grained } \\
\text { domain }\end{array}$ & & 0.8 & 0.03 & 0.7 & & 0.8 & 0.03 & 0.0 & 0.5 & 0.01 & & & & 0.8 & & 1.6 & \\
\hline \multirow[t]{3}{*}{$214 \mathrm{R}-2,78-81$} & 1413.13 & Gabbro 1 & $\mathrm{Pl}$ & $N=2$ & Subhedral & Core & 49.1 & 0.08 & 31.4 & - & 0.5 & - & 0.0 & 15.1 & 2.91 & - & - & - & 99.1 & 74.2 & & \\
\hline & & & & SD & & & 1.1 & & 1.0 & & 0.0 & & 0.0 & 1.0 & 0.70 & & & & 0.2 & 5.9 & & \\
\hline & & & $\mathrm{PI}$ & $N=1$ & Subhedral & $\operatorname{Rim}$ & 55.1 & 0.11 & 27.4 & - & 0.5 & - & - & 10.2 & 5.87 & - & - & - & 99.3 & 48.9 & & \\
\hline
\end{tabular}


Table T3 (continued). (Continued on next page.)

\begin{tabular}{|c|c|c|c|c|c|c|c|c|c|c|c|c|c|c|c|c|c|c|c|c|c|c|}
\hline \multirow{2}{*}{$\begin{array}{l}\text { Core, section, } \\
\text { interval (cm) }\end{array}$} & \multirow{2}{*}{$\begin{array}{l}\text { Depth } \\
\text { (mbsf) }\end{array}$} & \multirow[b]{2}{*}{ Rock name } & \multirow[b]{2}{*}{ Phase } & \multirow[b]{2}{*}{ Replicates } & \multirow{2}{*}{$\begin{array}{l}\text { Mode of } \\
\text { occurrence }\end{array}$} & \multirow[b]{2}{*}{ AP } & \multicolumn{16}{|c|}{ Major element oxide (wt\%) } \\
\hline & & & & & & & $\mathrm{SiO}_{2}$ & $\mathrm{TiO}_{2}$ & $\mathrm{Al}_{2} \mathrm{O}_{3}$ & $\mathrm{Cr}_{2} \mathrm{O}_{3}$ & $\mathrm{FeO}$ & $\mathrm{MnO}$ & $\mathrm{MgO}$ & $\mathrm{CaO}$ & $\mathrm{Na}_{2} \mathrm{O}$ & $\mathrm{K}_{2} \mathrm{O}$ & $\mathrm{NiO}$ & $\mathrm{BaO}$ & Total & An\% & Mg\# & Fo \\
\hline & & & Cpx & $N=6$ & Igneous-type & Core & 51.6 & 0.61 & 2.8 & 0.38 & 6.6 & 0.13 & 17.4 & 20.2 & 0.27 & - & - & - & 100.2 & & 82.4 & \\
\hline & & & & SD & $\begin{array}{l}\text { Subophitic } \\
\text { domain }\end{array}$ & & 0.3 & 0.05 & 0.2 & 0.10 & 0.3 & 0.03 & 0.3 & 0.5 & 0.01 & & & & 0.3 & & 0.6 & \\
\hline & & & Cpx & $N=6$ & Igneous-type & Core & 51.4 & 0.59 & 2.1 & - & 9.3 & 0.19 & 16.2 & 19.5 & 0.29 & - & - & - & 99.9 & & 75.5 & \\
\hline & & & & SD & $\begin{array}{l}\text { Coarse-grained } \\
\text { domain }\end{array}$ & & 0.4 & 0.05 & 0.3 & & 1.1 & 0.06 & 0.6 & 0.4 & 0.01 & & & & 0.3 & & 2.9 & \\
\hline \multirow[t]{4}{*}{$214 \mathrm{R}-3,18-21$} & 1413.99 & Gabbro 1 & Cpx & $N=6$ & Igneous-type & Core & 51.5 & 0.62 & 2.1 & - & 7.4 & 0.12 & 17.5 & 20.0 & 0.24 & - & - & - & 99.7 & & 80.9 & \\
\hline & & & & SD & $\begin{array}{c}\text { Subophitic } \\
\text { domain }\end{array}$ & & 0.5 & 0.18 & 0.5 & 0.04 & 0.2 & & 0.3 & 0.1 & 0.01 & & & & 0.3 & & 0.3 & \\
\hline & & & $\mathrm{Cpx}$ & $N=3$ & Igneous-type & Core & 50.8 & 0.87 & 1.6 & - & 13.1 & 0.30 & 14.5 & 18.9 & 0.24 & - & - & - & 100.0 & & 66.3 & \\
\hline & & & & SD & $\begin{array}{l}\text { Coarse-grained } \\
\text { domain }\end{array}$ & & 0.4 & 0.13 & 0.2 & & 0.8 & 0.07 & 0.5 & 0.6 & 0.02 & & & & 0.8 & & 1.9 & \\
\hline \multirow[t]{12}{*}{$215 R-1,20-23$} & 1415.92 & Gabbro 1 & $\mathrm{PI}$ & $\begin{array}{l}N=1 \\
S D\end{array}$ & Subhedral & Core & 48.7 & - & 31.6 & - & 0.3 & - & 0.1 & 15.5 & 2.70 & - & - & 0.17 & 99.1 & 76.0 & & \\
\hline & & & PI & $\begin{array}{l}N=1 \\
S D\end{array}$ & Subhedral & $\operatorname{Rim}$ & 49.5 & - & 30.9 & - & 0.4 & - & 0.1 & 14.9 & 3.13 & - & - & - & 99.2 & 72.5 & & \\
\hline & & & Cpx & $N=9$ & Igneous-type & Core & 51.8 & 0.54 & 2.4 & 0.22 & 6.7 & 0.22 & 17.7 & 20.2 & 0.22 & - & - & 0.16 & 100.2 & & 82.4 & \\
\hline & & & & SD & $\begin{array}{l}\text { Subophitic } \\
\text { domain }\end{array}$ & & 0.5 & 0.06 & 0.5 & 0.09 & 0.6 & 0.03 & 0.4 & 0.6 & 0.03 & & & 0.04 & 0.4 & & 1.3 & \\
\hline & & & $\mathrm{Cpx}$ & $N=3$ & Igneous-type & & 50.8 & 0.63 & 2.2 & & 11.1 & 0.32 & 16.4 & 17.6 & 0.20 & - & - & 0.19 & 99.6 & & 72.4 & \\
\hline & & & & SD & $\begin{array}{l}\text { Coarse-grained } \\
\text { domain }\end{array}$ & & 0.3 & 0.13 & 0.3 & & 2.0 & 0.05 & 0.9 & 0.8 & 0.02 & & & 0.04 & 1.2 & & 4.5 & \\
\hline & & & Opx & $N=8$ & Anhedral & & 51.8 & 0.43 & 0.6 & - & 23.7 & 0.52 & 21.4 & 2.1 & - & - & - & 0.17 & 100.9 & & 61.6 & \\
\hline & & & & SD & & & 0.3 & 0.05 & 0.3 & & 1.9 & 0.11 & 1.7 & 0.4 & & & & 0.05 & 0.5 & & 3.8 & \\
\hline & & & Ol & $N=3$ & Anhedral & Core & 38.7 & - & - & - & 20.4 & 0.28 & 42.5 & 0.0 & - & - & 0.12 & - & 102.1 & & & 78.8 \\
\hline & & & & SD & & & 0.1 & & & & 1.1 & 0.01 & 1.0 & 0.0 & & & 0.02 & & 0.4 & & & 1.3 \\
\hline & & & Ol & $N=2$ & Anhedral & $\operatorname{Rim}$ & 38.0 & - & - & - & 21.8 & 0.28 & 41.3 & 0.1 & - & - & 0.14 & - & 101.6 & & & 77.2 \\
\hline & & & & SD & & & 0.6 & & & & 0.6 & 0.02 & 0.6 & 0.0 & & & 0.00 & & 0.7 & & & 0.8 \\
\hline \multirow[t]{10}{*}{$215 R-2,56-59$} & 1417.69 & Gabbro 1 & $\mathrm{PI}$ & $N=3$ & Subhedral & Core & 50.6 & 0.07 & 30.8 & - & 0.6 & - & 0.1 & 14.1 & 3.45 & - & - & - & 99.8 & 69.3 & & \\
\hline & & & & SD & & & 1.9 & & 1.5 & & 0.0 & & 0.0 & 1.6 & 0.73 & & & & 0.5 & 7.0 & & \\
\hline & & & $\mathrm{Pl}$ & $N=3$ & Subhedral & $\operatorname{Rim}$ & 55.2 & - & 27.3 & - & 0.6 & - & 0.0 & 10.2 & 5.71 & 0.13 & - & - & 99.2 & 49.7 & & \\
\hline & & & & SD & & & 3.1 & & 2.1 & & 0.1 & & 0.0 & 2.4 & 1.34 & & & & 0.1 & 11.6 & & \\
\hline & & & Cpx & $N=7$ & Igneous-type & Core & 52.5 & 0.44 & 2.3 & 0.46 & 5.8 & 0.15 & 18.1 & 20.0 & 0.22 & - & - & - & 100.0 & & 84.7 & \\
\hline & & & & SD & & & 0.5 & 0.04 & 0.5 & 0.24 & 0.7 & 0.03 & 0.4 & 0.4 & 0.02 & & & & 0.5 & & 1.6 & \\
\hline & & & Opx & $N=10$ & Anhedral & & 52.8 & 0.38 & 0.8 & - & 23.2 & 0.46 & 22.2 & 2.2 & - & - & - & - & 102.1 & & 62.3 & \\
\hline & & & & SD & & & 0.6 & 0.10 & 0.1 & & 2.6 & 0.10 & 1.1 & 1.6 & & & & & 0.7 & & 2.9 & \\
\hline & & & Opx & $N=6$ & $\begin{array}{l}\text { Within olivine } \\
\text { pseudomorph }\end{array}$ & & 54.8 & 0.07 & 0.9 & - & 19.2 & 0.52 & 26.7 & 0.3 & - & - & - & - & 102.5 & & 71.2 & \\
\hline & & & & SD & & & 0.5 & & 0.5 & - & 0.6 & 0.08 & 0.5 & 0.1 & & & & & 0.5 & & 0.9 & \\
\hline \multirow[t]{4}{*}{ 216R-1, 72-75 } & 1418.62 & Gabbro 1 & $\mathrm{Cpx}$ & $N=9$ & Igneous-type & Core & 52.2 & 0.42 & 1.8 & 0.19 & 6.3 & 0.20 & 18.0 & 20.4 & 0.20 & - & - & 0.16 & 99.9 & & 83.7 & \\
\hline & & & & SD & & & 0.3 & 0.05 & 0.2 & 0.23 & 0.4 & 0.03 & 0.2 & 0.2 & 0.02 & & & 0.02 & 0.4 & & 0.9 & \\
\hline & & & Opx & $N=8$ & Anhedral & & 52.4 & 0.41 & 1.1 & - & 19.7 & 0.42 & 23.4 & 2.6 & - & - & - & 0.21 & 100.3 & & 67.9 & \\
\hline & & & & SD & & & 0.7 & 0.08 & 0.4 & & 1.3 & 0.04 & 0.6 & 0.6 & & & & 0.02 & 0.4 & & 1.9 & \\
\hline \multirow[t]{6}{*}{ 216R-1, 138-142 } & 1419.19 & Gabbro 1 & $\mathrm{Pl}$ & $N=8$ & Subhedral & Core & 49.8 & - & 31.2 & - & 0.5 & - & 0.0 & 15.2 & 2.98 & - & - & - & 99.8 & 73.9 & & \\
\hline & & & & SD & & & 1.1 & & 0.9 & & 0.1 & & 0.0 & 1.0 & 0.53 & & & & 0.5 & 4.6 & & \\
\hline & & & $\mathrm{Pl}$ & $N=9$ & Subhedral & $\operatorname{Rim}$ & 51.2 & - & 30.2 & - & 0.5 & - & 0.0 & 14.0 & 3.64 & 0.11 & - & - & 99.7 & 68.1 & & \\
\hline & & & & SD & & & 1.9 & & 1.3 & & 0.0 & & 0.0 & 1.5 & 0.85 & & & & 0.3 & 7.4 & & \\
\hline & & & Cpx & $N=10$ & Igneous-type & Core & 52.9 & 0.46 & 2.0 & 0.35 & 5.8 & - & 17.9 & 20.8 & 0.20 & - & - & - & 100.6 & & 84.5 & \\
\hline & & & & SD & & & 0.5 & 0.05 & 0.4 & 0.12 & 0.2 & & 0.3 & 0.3 & 0.02 & & & & 0.4 & & 0.5 & \\
\hline
\end{tabular}


Table T3 (continued). (Continued on next page.)

\begin{tabular}{|c|c|c|c|c|c|c|c|c|c|c|c|c|c|c|c|c|c|c|c|c|c|c|}
\hline \multirow{2}{*}{$\begin{array}{l}\text { Core, section, } \\
\text { interval }(\mathrm{cm})\end{array}$} & \multirow{2}{*}{$\begin{array}{l}\text { Depth } \\
\text { (mbsf) }\end{array}$} & \multirow[b]{2}{*}{ Rock name } & \multirow[b]{2}{*}{ Phase } & \multirow[b]{2}{*}{ Replicates } & \multirow{2}{*}{$\begin{array}{l}\text { Mode of } \\
\text { occurrence }\end{array}$} & \multirow[b]{2}{*}{ AP } & \multicolumn{16}{|c|}{ Major element oxide (wt\%) } \\
\hline & & & & & & & $\mathrm{SiO}_{2}$ & $\mathrm{TiO}_{2}$ & $\mathrm{Al}_{2} \mathrm{O}_{3}$ & $\mathrm{Cr}_{2} \mathrm{O}_{3}$ & $\mathrm{FeO}$ & $\mathrm{MnO}$ & $\mathrm{MgO}$ & $\mathrm{CaO}$ & $\mathrm{Na}_{2} \mathrm{O}$ & $\mathrm{K}_{2} \mathrm{O}$ & $\mathrm{NiO}$ & $\mathrm{BaO}$ & Total & An $\%$ & Mg\# & Fo \\
\hline \multirow{6}{*}{$217 \mathrm{R}-1,94-97$} & 1422.54 & Gabbro 1 & $\mathrm{PI}$ & $N=7$ & Subhedral & Core & 53.0 & - & 28.8 & - & 0.5 & - & 0.0 & 12.5 & 4.51 & 0.14 & - & - & 99.5 & 60.6 & & \\
\hline & & & & SD & & & 2.3 & & 1.4 & & 0.1 & & 0.0 & 1.7 & 1.03 & & & & 0.4 & 8.7 & & \\
\hline & & & $\mathrm{PI}$ & $N=3$ & Subhedral & $\operatorname{Rim}$ & 54.2 & - & 28.2 & - & 0.5 & - & - & 11.7 & 5.14 & - & - & - & 99.8 & 55.9 & & \\
\hline & & & & SD & & & 2.4 & & 1.4 & & 0.0 & & & 1.9 & 1.14 & & & & 0.4 & 9.6 & & \\
\hline & & & Cpx & $N=8$ & Igneous-type & Core & 52.5 & 0.48 & 2.3 & 0.49 & 5.5 & - & 17.7 & 20.9 & 0.21 & - & - & - & 100.1 & & 85.2 & \\
\hline & & & & SD & & & 0.4 & 0.06 & 0.6 & 0.14 & 0.5 & & 0.5 & 0.3 & 0.02 & & & & 0.5 & & 1.6 & \\
\hline \multirow[t]{2}{*}{$218 \mathrm{R}-1,1-3$} & 1430 & Gabbro 1 & Cpx & $N=9$ & Igneous-type & Core & 52.4 & 0.54 & 2.0 & 0.15 & 7.4 & - & 17.5 & 20.2 & 0.21 & - & - & - & 100.5 & & 80.8 & \\
\hline & & & & SD & & & 0.5 & 0.17 & 0.2 & 0.11 & 1.9 & 0.01 & 0.6 & 1.2 & 0.02 & & & & 0.4 & & 2.1 & \\
\hline \multirow[t]{12}{*}{$219 \mathrm{R}-1,5-8$} & 1430.05 & Gabbro 1 & $\mathrm{PI}$ & $N=7$ & Subhedral & Core & 50.2 & - & 30.7 & - & 0.5 & - & 0.0 & 14.6 & 3.39 & - & - & - & 99.5 & 70.4 & & \\
\hline & & & & SD & & & 0.9 & & 0.8 & & 0.1 & & 0.0 & 0.7 & 0.42 & & & & 0.4 & 3.6 & & \\
\hline & & & $\mathrm{Pl}$ & $N=6$ & Subhedral & $\operatorname{Rim}$ & 53.2 & 0.09 & 28.7 & - & 0.6 & - & 0.1 & 12.0 & 4.79 & - & - & - & 99.4 & 58.1 & & \\
\hline & & & & SD & & & 1.4 & & 1.1 & & 0.1 & & 0.0 & 1.0 & 0.62 & & & & 0.3 & 5.3 & & \\
\hline & & & Cpx & $N=6$ & Igneous-type & Core & 52.5 & 0.41 & 2.3 & 0.58 & 5.8 & - & 17.8 & 20.9 & 0.24 & - & - & - & 100.6 & & 84.6 & \\
\hline & & & & SD & & & 0.3 & 0.08 & 0.5 & 0.28 & 0.5 & & 0.3 & 0.2 & 0.03 & & & & 0.3 & & 1.2 & \\
\hline & & & Opx & $N=5$ & Anhedral & & 53.3 & 0.45 & 0.8 & - & 19.6 & 0.35 & 24.5 & 1.9 & - & - & - & - & 101.0 & & 69.1 & \\
\hline & & & & SD & & & 0.4 & 0.05 & 0.1 & & 1.0 & 0.06 & 0.6 & 0.1 & & & & & 0.8 & & 1.6 & \\
\hline & & & Ol & $N=5$ & Anhedral & Core & 36.9 & - & - & - & 30.3 & 0.40 & 34.7 & 0.1 & - & - & 0.08 & - & 102.4 & & & 67.1 \\
\hline & & & & SD & & & 0.4 & & & & 0.7 & 0.01 & 0.7 & 0.0 & & & 0.01 & & 0.4 & & & 0.9 \\
\hline & & & $\mathrm{Ol}$ & $N=5$ & Anhedral & $\operatorname{Rim}$ & 37.0 & - & - & - & 31.2 & 0.42 & 33.9 & 0.0 & - & - & 0.08 & - & 102.6 & & & 66.0 \\
\hline & & & & SD & & & 0.4 & & & & 0.8 & 0.02 & 0.4 & 0.0 & & & 0.01 & & 0.8 & & & 0.8 \\
\hline \multirow[t]{12}{*}{$222 \mathrm{R}-1,73-78$} & 1445.33 & Gabbro 1 & $\mathrm{PI}$ & $N=7$ & Subhedral & Core & 50.1 & - & 30.8 & - & 0.6 & - & 0.1 & 14.7 & 3.34 & - & - & - & 99.5 & 70.9 & & \\
\hline & & & & SD & & & 1.4 & & 1.0 & & 0.1 & & 0.0 & 1.2 & 0.66 & & & & 0.2 & 5.7 & & \\
\hline & & & $\mathrm{PI}$ & $N=5$ & Subhedral & Rim & 52.0 & 0.09 & 29.6 & - & 0.6 & - & 0.0 & 13.2 & 4.22 & - & - & - & 99.7 & 63.3 & & \\
\hline & & & & SD & & & 1.2 & 0.01 & 0.8 & & 0.1 & & 0.0 & 1.1 & 0.62 & & & & 0.5 & 5.4 & & \\
\hline & & & Cpx & $N=6$ & Igneous-type & Core & 52.3 & 0.46 & 2.6 & 0.60 & 5.3 & - & 17.7 & 20.8 & 0.25 & - & - & - & 100.1 & & 85.6 & \\
\hline & & & & SD & & & 0.2 & 0.07 & 0.3 & 0.14 & 0.1 & & 0.1 & 0.1 & 0.02 & & & & 0.3 & & 0.4 & \\
\hline & & & Opx & $N=7$ & Anhedral & & 53.5 & 0.62 & 1.2 & - & 16.5 & 0.29 & 26.3 & 2.1 & - & - & - & - & 100.6 & & 74.0 & \\
\hline & & & & SD & & & 0.2 & 0.11 & 0.1 & & 0.5 & 0.04 & 0.2 & 0.2 & 0.01 & & & & 0.5 & & 0.6 & \\
\hline & & & Ol & $N=3$ & Subhedral & Core & 37.8 & - & - & - & 27.8 & 0.38 & 36.6 & 0.0 & - & - & 0.10 & - & 102.8 & & & 70.1 \\
\hline & & & & SD & & & 0.9 & & & & 0.1 & 0.01 & 0.0 & 0.0 & & & 0.00 & & 1.0 & & & 0.1 \\
\hline & & & Ol & $N=3$ & Subhedral & $\operatorname{Rim}$ & 37.6 & - & 0.1 & - & 28.1 & 0.35 & 36.4 & 0.0 & - & - & 0.10 & - & 102.7 & & & 69.8 \\
\hline & & & & SD & & & 1.0 & & 0.1 & & 0.3 & 0.01 & 0.3 & 0.0 & & & 0.01 & & 1.3 & & & 0.4 \\
\hline \multirow[t]{12}{*}{$222 \mathrm{R}-2,60-63$} & 1446.70 & Gabbro 1 & $\mathrm{Pl}$ & $N=4$ & Subhedral & Core & 50.0 & - & 31.6 & - & 0.5 & - & 0.0 & 15.0 & 3.08 & - & - & - & 100.2 & 72.9 & & \\
\hline & & & & SD & & & 1.3 & & 0.9 & & 0.1 & & 0.0 & 1.3 & 0.61 & & & & 0.3 & 5.6 & & \\
\hline & & & $\mathrm{Pl}$ & $N=5$ & Subhedral & $\operatorname{Rim}$ & 51.8 & 0.10 & 30.4 & - & 0.5 & - & 0.0 & 13.5 & 3.92 & - & - & - & 100.2 & 65.6 & & \\
\hline & & & & SD & & & 1.5 & 0.02 & 1.1 & & 0.1 & & 0.0 & 1.2 & 0.77 & & & & 0.4 & 6.4 & & \\
\hline & & & Cpx & $N=4$ & Igneous-type & Core & 52.3 & 0.42 & 2.7 & 0.85 & 5.7 & - & 17.3 & 21.4 & 0.29 & - & - & - & 101.0 & & 84.4 & \\
\hline & & & & SD & & & 0.3 & 0.03 & 0.3 & 0.14 & 0.5 & & 0.2 & 0.0 & 0.04 & & & & 0.2 & & 1.3 & \\
\hline & & & Opx & $N=4$ & Anhedral & & 53.8 & 0.45 & 0.9 & - & 17.9 & 0.37 & 25.8 & 1.9 & - & - & - & - & 101.2 & & 72.0 & \\
\hline & & & & SD & & & 0.1 & 0.03 & 0.1 & & 0.4 & 0.06 & 0.2 & 0.1 & & & & & 0.5 & & 0.3 & \\
\hline & & & Ol & $N=5$ & Subhedral & Core & 37.3 & - & - & - & 30.3 & 0.37 & 34.6 & 0.0 & - & - & 0.08 & - & 102.7 & & & 67.1 \\
\hline & & & & SD & & & 0.6 & & & & 0.2 & 0.01 & 0.1 & 0.0 & & & 0.01 & & 0.8 & & & 0.2 \\
\hline & & & $\mathrm{Ol}$ & $N=5$ & Subhedral & $\operatorname{Rim}$ & 36.8 & - & - & - & 30.4 & 0.38 & 34.5 & 0.0 & - & - & 0.08 & - & 102.3 & & & 67.0 \\
\hline & & & & SD & & & 0.7 & & & & 0.4 & 0.00 & 0.4 & 0.0 & & & 0.00 & & 0.9 & & & 0.4 \\
\hline 223R-1, 8-12 & 1449.37 & Gabbro 1 & $\mathrm{PI}$ & $N=5$ & Subhedral & Core & 51.0 & - & 30.2 & - & 0.5 & - & 0.0 & 13.8 & 3.79 & - & - & - & 99.5 & 66.9 & & \\
\hline & & & & SD & & & 1.3 & & 1.0 & & 0.1 & & 0.0 & 1.1 & 0.71 & & & & 0.3 & 6.0 & & \\
\hline & & & $\mathrm{PI}$ & $N=5$ & Subhedral & $\operatorname{Rim}$ & 51.6 & - & 30.0 & - & 0.6 & - & 0.0 & 13.4 & 4.07 & - & - & - & 99.7 & 64.5 & & \\
\hline & & & & SD & & & 0.9 & & 0.8 & & 0.1 & & 0.0 & 0.9 & 0.49 & & & & 0.3 & 4.3 & & \\
\hline
\end{tabular}


Table T3 (continued). (Continued on next page.)

\begin{tabular}{|c|c|c|c|c|c|c|c|c|c|c|c|c|c|c|c|c|c|c|c|c|c|c|}
\hline \multirow{2}{*}{$\begin{array}{l}\text { Core, section, } \\
\text { interval }(\mathrm{cm})\end{array}$} & \multirow{2}{*}{$\begin{array}{l}\text { Depth } \\
\text { (mbsf) }\end{array}$} & \multirow[b]{2}{*}{ Rock name } & \multirow[b]{2}{*}{ Phase } & \multirow[b]{2}{*}{ Replicates } & \multirow{2}{*}{$\begin{array}{l}\text { Mode of } \\
\text { occurrence }\end{array}$} & \multirow[b]{2}{*}{ AP } & \multicolumn{16}{|c|}{ Major element oxide (wt\%) } \\
\hline & & & & & & & $\mathrm{SiO}_{2}$ & $\mathrm{TiO}_{2}$ & $\mathrm{Al}_{2} \mathrm{O}_{3}$ & $\mathrm{Cr}_{2} \mathrm{O}_{3}$ & $\mathrm{FeO}$ & $\mathrm{MnO}$ & $\mathrm{MgO}$ & $\mathrm{CaO}$ & $\mathrm{Na}_{2} \mathrm{O}$ & $\mathrm{K}_{2} \mathrm{O}$ & $\mathrm{NiO}$ & $\mathrm{BaO}$ & Total & An\% & Mg\# & Fo \\
\hline & & & Cpx & $N=5$ & Igneous-type & Core & 52.6 & 0.39 & 2.1 & 0.33 & 6.1 & 0.11 & 17.8 & 21.0 & 0.20 & - & - & - & 100.7 & & 83.9 & \\
\hline & & & & SD & & & 0.2 & 0.04 & 0.1 & 0.08 & 0.2 & 0.01 & 0.3 & 0.2 & 0.02 & & & & 0.3 & & 0.6 & \\
\hline & & & Opx & $N=8$ & Anhedral & & 53.3 & 0.54 & 1.1 & - & 17.3 & 0.32 & 25.9 & 1.9 & - & - & - & - & 100.6 & & 72.7 & \\
\hline & & & & SD & & & 0.3 & 0.07 & 0.2 & & 0.4 & 0.02 & 0.3 & 0.1 & & & & & 0.5 & & 0.4 & \\
\hline & & & Ol & $N=5$ & Subhedral & Core & 38.4 & - & - & - & 29.2 & 0.38 & 34.7 & 0.2 & - & - & 0.08 & - & 103.0 & & & 67.9 \\
\hline & & & & SD & & & 0.3 & & & & 0.5 & 0.01 & 0.2 & 0.3 & & & 0.00 & & 0.3 & & & 0.5 \\
\hline & & & Ol & $N=5$ & Subhedral & $\operatorname{Rim}$ & 38.3 & - & - & - & 29.9 & 0.37 & 34.2 & 0.1 & - & - & 0.08 & - & 103.0 & & & 67.1 \\
\hline & & & & SD & & & 0.3 & & & & 0.5 & 0.02 & 0.6 & 0.0 & & & 0.02 & & 0.3 & & & 0.8 \\
\hline \multirow[t]{18}{*}{ 223R-2, 133-137 } & 1452.11 & Gabbro 1 & $\mathrm{PI}$ & $N=4$ & Subhedral & Core & 52.2 & - & 29.5 & - & 0.5 & - & 0.0 & 12.9 & 4.34 & - & - & - & 99.6 & 62.1 & & \\
\hline & & & & SD & & & 0.4 & & 0.5 & & 0.1 & & 0.0 & 0.3 & 0.25 & & & & 0.3 & 1.9 & & \\
\hline & & & $\mathrm{PI}$ & $N=5$ & Subhedral & $\operatorname{Rim}$ & 52.8 & - & 29.1 & - & 0.7 & - & 0.0 & 12.3 & 4.68 & - & - & - & 99.6 & 59.1 & & \\
\hline & & & & SD & & & 0.3 & & 0.2 & & 0.0 & & 0.0 & 0.2 & 0.06 & & & & 0.5 & 0.7 & & \\
\hline & & & $\mathrm{PI}$ & $N=7$ & Microgranular & Core & 50.1 & - & 31.2 & - & 0.3 & - & 0.0 & 14.6 & 3.29 & - & - & - & 99.5 & 71.0 & & \\
\hline & & & & SD & & & 1.7 & & 1.0 & & 0.1 & & 0.0 & 1.4 & 0.76 & & & & 0.3 & 6.7 & & \\
\hline & & & Cpx & $N=3$ & Igneous-type & Core & 51.6 & 0.62 & 1.9 & - & 8.3 & 0.18 & 15.2 & 21.8 & 0.34 & - & - & - & 100.3 & & 76.5 & \\
\hline & & & & SD & & & 0.2 & 0.11 & 0.1 & & 0.2 & 0.01 & 0.1 & 0.1 & 0.02 & & & & 0.3 & & 0.3 & \\
\hline & & & Cpx & $N=6$ & Secondary-type & Core & 53.4 & - & 0.4 & - & 6.9 & 0.20 & 15.3 & 24.2 & 0.09 & - & - & - & 100.6 & & 79.8 & \\
\hline & & & & SD & & & 0.3 & & 0.2 & & 0.7 & 0.04 & 0.4 & 0.8 & 0.04 & & & & 0.5 & & 2.0 & \\
\hline & & & $C p x$ & $N=7$ & Microgranular & Core & 51.7 & 0.68 & 2.1 & 0.30 & 9.1 & 0.23 & 16.0 & 19.6 & 0.32 & - & - & - & 100.0 & & 75.8 & \\
\hline & & & & SD & & & 0.3 & 0.13 & 0.1 & 0.10 & 0.4 & 0.04 & 0.4 & 0.8 & 0.03 & & & & 0.6 & & 0.8 & \\
\hline & & & Opx & $N=8$ & Anhedral & & 53.3 & 0.54 & 1.2 & - & 16.9 & 0.32 & 26.0 & 2.2 & - & - & - & - & 100.6 & & 73.2 & \\
\hline & & & & SD & & & 0.3 & 0.05 & 0.1 & & 0.6 & 0.04 & 0.3 & 0.3 & & & & & 0.6 & & 0.8 & \\
\hline & & & Ol & $N=8$ & Interstitial & Core & 38.0 & 0.05 & - & - & 29.1 & 0.37 & 34.4 & 0.1 & & & 0.07 & & 102.0 & & & 67.8 \\
\hline & & & & SD & & & 0.2 & 0.01 & - & - & 0.2 & 0.03 & 0.2 & 0.0 & & & 0.01 & & 0.3 & & & 0.3 \\
\hline & & & Ol & $N=5$ & Interstitial & $\operatorname{Rim}$ & 38.0 & 0.06 & - & - & 29.1 & 0.37 & 34.3 & 0.1 & & & 0.07 & & 101.9 & & & 67.7 \\
\hline & & & & SD & & & 0.2 & 0.00 & - & - & 0.4 & 0.03 & 0.4 & 0.0 & & & 0.01 & & 0.4 & & & 0.5 \\
\hline \multirow[t]{6}{*}{$226 \mathrm{R}-1,0-4$} & 1463.90 & Type 8 & $\mathrm{Pl}$ & $N=2$ & Subhedral & Core & 52.5 & - & 29.6 & - & 0.4 & - & - & 12.5 & 4.37 & - & - & - & 99.5 & 61.4 & & \\
\hline & & UD & & SD & & & 0.3 & & 0.0 & & 0.1 & & & 0.4 & 0.06 & & & & 0.0 & 0.4 & & \\
\hline & & & PI & $N=3$ & Subhedral & $\operatorname{Rim}$ & 54.5 & - & 28.3 & - & 0.4 & - & - & 11.2 & 5.38 & - & - & - & 100.0 & 53.5 & & \\
\hline & & & & SD & & & 1.4 & & 0.7 & & 0.0 & & & 0.9 & 0.54 & & & & 0.3 & 4.5 & & \\
\hline & & & $C p x$ & $N=7$ & Secondary-type & Core & 52.5 & 0.16 & 0.6 & - & 10.0 & 0.23 & 14.4 & 22.1 & 0.24 & - & - & - & 100.4 & & 71.9 & \\
\hline & & & & SD & & & 0.3 & 0.11 & 0.2 & & 0.4 & 0.04 & 0.3 & 0.7 & 0.06 & & & & 0.3 & & 0.8 & \\
\hline \multirow[t]{8}{*}{ 230R-1, 19-21 } & 1483.19 & Gabbro 2 & $\mathrm{PI}$ & $N=7$ & Subhedral & Core & 54.3 & - & 28.3 & - & 0.6 & - & 0.1 & 11.0 & 5.27 & - & - & - & 99.6 & 53.6 & & \\
\hline & & & & SD & & & 0.5 & & 0.3 & & 0.1 & & 0.0 & 0.5 & 0.24 & & & & 0.4 & 2.2 & & \\
\hline & & & $\mathrm{Pl}$ & $N=5$ & Subhedral & $\operatorname{Rim}$ & 55.7 & - & 27.3 & - & 0.6 & - & 0.0 & 10.0 & 6.00 & - & - & - & 99.7 & 47.9 & & \\
\hline & & & & SD & & & 1.7 & & 0.9 & & 0.1 & & 0.0 & 1.0 & 0.68 & & & & 0.5 & 5.4 & & \\
\hline & & & $\mathrm{Cpx}$ & $N=7$ & Amphibole-type & Core & 52.5 & 0.16 & 0.6 & - & 10.0 & 0.23 & 14.4 & 22.1 & 0.24 & - & - & - & 100.4 & & 71.9 & \\
\hline & & & & SD & & & 0.3 & 0.07 & 0.2 & & 0.4 & 0.04 & 0.3 & 0.7 & 0.06 & & & & 0.3 & & 0.8 & \\
\hline & & & Opx & $N=9$ & granular & & 52.6 & 0.35 & 0.8 & - & 22.5 & 0.46 & 22.2 & 1.9 & - & - & - & - & 100.9 & & 63.7 & \\
\hline & & & & SD & & & 0.2 & 0.08 & 0.2 & & 0.6 & 0.04 & 0.3 & 0.3 & & & & & 0.4 & & 0.8 & \\
\hline \multirow{7}{*}{ 230R-1, 81-84 } & 1483.81 & Gabbro 2 & $\mathrm{PI}$ & $N=5$ & Subhedral & Core & 54.3 & 0.09 & 28.2 & - & 0.5 & - & 0.1 & 11.1 & 5.40 & - & - & - & 99.7 & 53.3 & & \\
\hline & & & & SD & & & 0.5 & 0.01 & 0.2 & & 0.2 & & 0.1 & 0.2 & 0.20 & & & & 0.4 & 1.4 & & \\
\hline & & & $\mathrm{Pl}$ & $N=5$ & Subhedral & $\operatorname{Rim}$ & 56.7 & - & 26.7 & - & 0.4 & - & - & 9.2 & 6.35 & - & - & - & 99.4 & 44.3 & & \\
\hline & & & & SD & & & 1.5 & & 1.4 & & 0.1 & & & 1.7 & 0.87 & & & & 0.8 & 8.0 & & \\
\hline & & & $\mathrm{Cpx}$ & $N=3$ & Igneous-type & Core & 51.7 & 0.57 & 1.3 & - & 10.6 & 0.26 & 14.9 & 20.6 & 0.34 & - & - & - & 100.5 & & 71.6 & \\
\hline & & & & SD & & & 0.4 & 0.09 & 0.3 & & 0.4 & 0.03 & 0.1 & 0.4 & 0.04 & & & & 0.6 & & 0.9 & \\
\hline & & & $\mathrm{Cpx}$ & $N=6$ & Secondary-type & Core & 53.2 & 0.07 & 0.3 & - & 8.4 & 0.15 & 14.3 & 22.9 & 0.64 & - & - & - & 100.2 & & 75.2 & \\
\hline
\end{tabular}


Table T3 (continued). (Continued on next page.)

\begin{tabular}{|c|c|c|c|c|c|c|c|c|c|c|c|c|c|c|c|c|c|c|c|c|c|c|}
\hline \multirow{2}{*}{$\begin{array}{l}\text { Core, section, } \\
\text { interval (cm) }\end{array}$} & \multirow{2}{*}{$\begin{array}{l}\text { Depth } \\
\text { (mbsf) }\end{array}$} & \multirow[b]{2}{*}{ Rock name } & \multirow[b]{2}{*}{ Phase } & \multirow[b]{2}{*}{ Replicates } & \multirow{2}{*}{$\begin{array}{l}\text { Mode of } \\
\text { occurrence }\end{array}$} & \multirow[b]{2}{*}{ AP } & \multicolumn{16}{|c|}{ Major element oxide (wt\%) } \\
\hline & & & & & & & $\mathrm{SiO}_{2}$ & $\mathrm{TiO}_{2}$ & $\mathrm{Al}_{2} \mathrm{O}_{3}$ & $\mathrm{Cr}_{2} \mathrm{O}_{3}$ & $\mathrm{FeO}$ & $\mathrm{MnO}$ & $\mathrm{MgO}$ & $\mathrm{CaO}$ & $\mathrm{Na}_{2} \mathrm{O}$ & $\mathrm{K}_{2} \mathrm{O}$ & $\mathrm{NiO}$ & $\mathrm{BaO}$ & Total & An\% & Mg\# & Fo \\
\hline & & & & SD & & & 0.3 & 0.01 & 0.1 & & 0.9 & 0.05 & 0.4 & 0.3 & 0.13 & & & & 0.4 & & 2.4 & \\
\hline & & & Opx & $N=16$ & granular & & 52.5 & 0.39 & 0.8 & - & 22.1 & 0.45 & 22.4 & 1.9 & - & - & - & - & 100.9 & & 64.4 & \\
\hline & & & & SD & & & 0.2 & 0.03 & 0.1 & & 0.7 & 0.05 & 0.5 & 0.1 & & & & & 0.6 & & 1.1 & \\
\hline \multirow{8}{*}{ 230R-1, 139-142 } & 1484.38 & Gabbro 2 & $\mathrm{PI}$ & $N=5$ & Subhedral & Core & 53.2 & 0.09 & 28.7 & - & 0.7 & 0.11 & 0.1 & 12.0 & 4.79 & - & - & - & 99.7 & 58.0 & & \\
\hline & & & & SD & & & 0.8 & 0.00 & 0.7 & & 0.1 & 0.00 & 0.0 & 0.7 & 0.44 & & & & 0.3 & 3.7 & & \\
\hline & & & $\mathrm{PI}$ & $N=3$ & Subhedral & $\operatorname{Rim}$ & 53.8 & 0.08 & 28.2 & - & 0.7 & - & 0.1 & 11.5 & 5.10 & - & - & - & 99.6 & 55.4 & & \\
\hline & & & & SD & & & 0.8 & 0.01 & 0.8 & & 0.1 & & 0.0 & 0.9 & 0.44 & & & & 0.3 & 4.0 & & \\
\hline & & & $C p x$ & $N=6$ & Igneous-type & Core & 52.8 & 0.07 & 0.4 & - & 10.1 & 0.27 & 14.0 & 22.7 & 0.24 & - & - & - & 100.7 & & 71.2 & \\
\hline & & & & SD & & & 0.3 & 0.04 & 0.2 & & 0.6 & 0.02 & 0.2 & 0.4 & 0.06 & & & & 0.4 & & 1.5 & \\
\hline & & & Cpx & $N=6$ & Amphibole-type & Core & 51.9 & 0.52 & 1.2 & - & 10.2 & 0.25 & 14.7 & 20.9 & 0.41 & - & - & - & 100.3 & & 72.1 & \\
\hline & & & & SD & & & 0.7 & 0.21 & 0.5 & & 1.0 & 0.04 & 0.4 & 0.9 & 0.17 & & & & 0.6 & & 1.7 & \\
\hline \multirow[t]{10}{*}{$230 \mathrm{R}-2,32-36$} & 1484.58 & Gabbro 2 & $\mathrm{PI}$ & $N=5$ & Subhedral & Core & 51.2 & - & 30.2 & - & 0.6 & - & 0.1 & 13.6 & 3.91 & - & - & - & 99.7 & 65.8 & & \\
\hline & & & & SD & & & 0.4 & & 0.4 & & 0.1 & & 0.0 & 0.5 & 0.19 & & & & 0.4 & 1.9 & & \\
\hline & & & $\mathrm{PI}$ & $N=3$ & Subhedral & $\operatorname{Rim}$ & 53.1 & - & 29.0 & - & 0.5 & - & 0.0 & 11.9 & 4.79 & 0.08 & - & - & 99.5 & 57.9 & & \\
\hline & & & & SD & & & 0.2 & & 0.1 & & 0.1 & & 0.0 & 0.2 & 0.15 & & & & 0.1 & 1.2 & & \\
\hline & & & $C p x$ & $N=6$ & Igneous-type & Core & 52.1 & 0.30 & 0.9 & - & 10.3 & 0.34 & 14.6 & 21.6 & 0.30 & - & - & - & 100.6 & & 71.7 & \\
\hline & & & & SD & & & 0.3 & 0.03 & 0.3 & & 0.5 & 0.05 & 0.3 & 0.3 & 0.05 & & & & 0.3 & & 1.3 & \\
\hline & & & Cpx & $N=6$ & Amphibole-type & Core & 52.8 & 0.07 & 0.4 & - & 10.1 & 0.27 & 14.0 & 22.7 & 0.24 & - & - & - & 100.7 & & 71.2 & \\
\hline & & & & SD & & & 0.3 & 0.04 & 0.2 & & 0.6 & 0.02 & 0.2 & 0.4 & 0.06 & & & & 0.4 & & 1.5 & \\
\hline & & & Opx & $N=13$ & Anhedral & & 52.7 & 0.50 & 0.8 & - & 21.8 & 0.54 & 22.9 & 2.0 & - & - & - & - & 101.3 & & 65.2 & \\
\hline & & & & SD & & & 0.4 & 0.07 & 0.2 & & 0.9 & 0.04 & 0.5 & 0.2 & & & & & 0.5 & & 1.4 & \\
\hline \multirow[t]{10}{*}{ 230R-2, 104-109 } & 1485.54 & Gabbro 2 & $\mathrm{PI}$ & $N=5$ & Subhedral & Core & 52.1 & - & 29.4 & - & 0.6 & - & 0.1 & 12.8 & 4.30 & - & - & - & 99.4 & 62.3 & & \\
\hline & & & & SD & & & 0.8 & & 0.8 & & 0.1 & & 0.0 & 0.7 & 0.49 & & & & 0.4 & 3.9 & & \\
\hline & & & $\mathrm{PI}$ & $N=3$ & Subhedral & $\operatorname{Rim}$ & 53.1 & - & 28.8 & - & 0.6 & - & 0.0 & 12.1 & 4.72 & - & - & - & 99.5 & 58.6 & & \\
\hline & & & & SD & & & 1.4 & & 1.0 & & 0.1 & & 0.0 & 1.4 & 0.77 & & & & 0.3 & 6.8 & & \\
\hline & & & Cpx & $N=14$ & Igneous-type & Core & 52.1 & 0.40 & 1.1 & - & 10.2 & 0.22 & 14.7 & 21.5 & 0.27 & - & - & - & 100.7 & & 72.0 & \\
\hline & & & & SD & & & 0.6 & 0.23 & 0.5 & & 1.4 & 0.06 & 0.6 & 1.5 & 0.03 & & & & 0.6 & & 2.8 & \\
\hline & & & $C p x$ & $N=2$ & Amphibole-type & Core & 52.4 & 0.20 & 1.0 & - & 10.0 & 0.22 & 14.3 & 21.7 & 0.28 & - & - & - & 100.3 & & 72.0 & \\
\hline & & & & SD & & & 0.5 & 0.00 & 0.5 & & 1.3 & 0.01 & 0.0 & 1.2 & 0.02 & & & & 0.0 & & 2.6 & \\
\hline & & & Opx & $N=8$ & Anhedral & & 53.0 & 0.49 & 0.9 & - & 21.1 & 0.39 & 23.8 & 2.0 & - & - & - & - & 101.8 & & 66.9 & \\
\hline & & & & SD & & & 0.3 & 0.05 & 0.1 & & 1.1 & 0.03 & 0.6 & 0.1 & & & & & 0.5 & & 1.7 & \\
\hline \multirow[t]{10}{*}{$231 \mathrm{R}-1,19-22$} & 1487.90 & Gabbro 2 & $\mathrm{PI}$ & $N=7$ & Subhedral & Core & 50.4 & 0.09 & 30.7 & - & 0.6 & - & 0.0 & 14.1 & 3.54 & 0.08 & 0.22 & - & 99.5 & 68.7 & & \\
\hline & & & & SD & & & 0.3 & 0.02 & 0.2 & & 0.1 & & 0.0 & 0.5 & 0.22 & & & & 0.4 & 2.1 & & \\
\hline & & & $\mathrm{PI}$ & $N=5$ & Subhedral & $\operatorname{Rim}$ & 52.7 & 0.07 & 29.2 & - & 0.5 & - & 0.0 & 12.1 & 4.69 & 0.08 & - & - & 99.5 & 58.8 & & \\
\hline & & & & SD & & & 1.5 & 0.00 & 0.9 & & 0.1 & & 0.0 & 1.4 & 0.68 & 0.00 & & & 0.2 & 6.2 & & \\
\hline & & & Cpx & $N=7$ & Igneous-type & Core & 51.9 & 0.57 & 1.7 & - & 10.2 & 0.21 & 15.7 & 20.3 & 0.21 & - & - & - & 101.0 & & 73.4 & \\
\hline & & & & SD & & & 0.5 & 0.08 & 0.4 & & 2.1 & 0.08 & 1.4 & 0.6 & 0.03 & & & & 0.3 & & 5.6 & \\
\hline & & & $C p x$ & $N=4$ & Amphibole-type & Core & 52.5 & 0.24 & 1.1 & - & 9.3 & 0.24 & 14.8 & 21.7 & 0.30 & - & - & - & 100.3 & & 73.9 & \\
\hline & & & & SD & & & 0.5 & 0.11 & 0.6 & & 0.2 & 0.03 & 0.2 & 1.0 & 0.06 & & & & 0.8 & & 0.3 & \\
\hline & & & Opx & $N=7$ & Anhedral & & 52.9 & 0.56 & 0.9 & - & 20.5 & 0.39 & 24.2 & 2.0 & - & - & - & - & 101.8 & & 67.8 & \\
\hline & & & & SD & & & 0.2 & 0.03 & 0.0 & & 0.6 & 0.05 & 0.3 & 0.0 & & & & & 0.8 & & 0.6 & \\
\hline \multirow[t]{7}{*}{$231 \mathrm{R}-2,35-39$} & 1489.54 & Gabbro 2 & $\mathrm{Pl}$ & $N=5$ & Subhedral & Core & 50.6 & 0.08 & 30.4 & - & 0.5 & - & 0.0 & 13.8 & 3.68 & - & - & - & 99.2 & 67.5 & & \\
\hline & & & & SD & & & 1.4 & & 0.9 & & 0.1 & & 0.0 & 1.2 & 0.70 & & & & 0.3 & 6.1 & & \\
\hline & & & $\mathrm{PI}$ & $N=5$ & Subhedral & $\operatorname{Rim}$ & 52.9 & - & 28.8 & - & 0.5 & - & 0.1 & 11.9 & 4.87 & - & - & - & 99.1 & 57.4 & & \\
\hline & & & & SD & & & 0.6 & & 0.4 & & 0.1 & & 0.1 & 0.6 & 0.27 & & & & 0.1 & 2.5 & & \\
\hline & & & Cpx & $N=15$ & Igneous-type & Core & 51.2 & 0.68 & 1.7 & 0.15 & 10.3 & 0.23 & 15.1 & 20.0 & 0.27 & - & - & - & 99.8 & & 72.4 & \\
\hline & & & & SD & & & 0.4 & 0.14 & 0.3 & 0.05 & 1.2 & 0.07 & 0.7 & 0.8 & 0.04 & & & & 0.7 & & 3.0 & \\
\hline & & & Cpx & $N=6$ & Amphibole-type & Core & 51.7 & 0.36 & 1.9 & 0.32 & 9.0 & 0.20 & 14.8 & 21.4 & 0.26 & - & - & - & 100.0 & & 74.5 & \\
\hline
\end{tabular}


Table T3 (continued). (Continued on next page.)

\begin{tabular}{|c|c|c|c|c|c|c|c|c|c|c|c|c|c|c|c|c|c|c|c|c|c|c|}
\hline \multirow{2}{*}{$\begin{array}{l}\text { Core, section, } \\
\text { interval }(\mathrm{cm})\end{array}$} & \multirow{2}{*}{$\begin{array}{l}\text { Depth } \\
\text { (mbsf) }\end{array}$} & \multirow[b]{2}{*}{ Rock name } & \multirow[b]{2}{*}{ Phase } & \multirow[b]{2}{*}{ Replicates } & \multirow{2}{*}{$\begin{array}{l}\text { Mode of } \\
\text { occurrence }\end{array}$} & \multirow[b]{2}{*}{ AP } & \multicolumn{16}{|c|}{ Major element oxide (wt\%) } \\
\hline & & & & & & & $\mathrm{SiO}_{2}$ & $\mathrm{TiO}_{2}$ & $\mathrm{Al}_{2} \mathrm{O}_{3}$ & $\mathrm{Cr}_{2} \mathrm{O}_{3}$ & $\mathrm{FeO}$ & $\mathrm{MnO}$ & $\mathrm{MgO}$ & $\mathrm{CaO}$ & $\mathrm{Na}_{2} \mathrm{O}$ & $\mathrm{K}_{2} \mathrm{O}$ & $\mathrm{NiO}$ & $\mathrm{BaO}$ & Total & $\mathrm{An} \%$ & Mg\# & Fo \\
\hline & & & & SD & & & 0.5 & 0.20 & 0.8 & 0.11 & 1.6 & 0.06 & 1.0 & 1.3 & 0.03 & & & & 0.3 & & 4.4 & \\
\hline & & & Opx & $N=9$ & Anhedral & & 52.6 & 0.50 & 0.9 & - & 20.0 & 0.39 & 23.6 & 2.1 & - & - & - & - & 100.3 & & 67.7 & \\
\hline & & & & SD & & & 0.3 & 0.05 & 0.1 & & 0.7 & 0.05 & 0.3 & 0.1 & & & & & 0.4 & & 1.0 & \\
\hline \multirow[t]{10}{*}{$231 \mathrm{R}-2,95-98$} & 1490.14 & Gabbro 2 & $\mathrm{Pl}$ & $N=3$ & Subhedral & Core & 50.2 & 0.08 & 30.9 & - & 0.6 & - & 0.1 & 14.3 & 3.40 & - & - & - & 99.6 & 69.9 & & \\
\hline & & & & SD & & & 0.5 & & 0.1 & & 0.1 & & 0.0 & 0.4 & 0.17 & & & & 0.4 & 1.6 & & \\
\hline & & & $\mathrm{PI}$ & $N=2$ & Subhedral & $\operatorname{Rim}$ & 53.2 & 0.09 & 28.6 & - & 0.7 & - & 0.1 & 11.4 & 4.94 & - & - & - & 99.1 & 56.1 & & \\
\hline & & & & SD & & & 0.9 & 0.02 & 0.5 & & 0.2 & & 0.0 & 0.8 & 0.46 & & & & 0.2 & 4.0 & & \\
\hline & & & $\mathrm{Cpx}$ & $N=8$ & Igneous-type & Core & 51.9 & 0.55 & 2.2 & 0.23 & 7.2 & 0.14 & 17.3 & 20.5 & 0.25 & - & - & - & 100.5 & & 81.0 & \\
\hline & & & & SD & & & 0.4 & 0.12 & 0.2 & 0.18 & 1.2 & 0.03 & 0.9 & 0.5 & 0.04 & & & & 0.3 & & 3.4 & \\
\hline & & & $C p x$ & $N=5$ & Amphibole-type & Core & 52.4 & 0.16 & 0.6 & - & 10.0 & 0.22 & 14.4 & 22.1 & 0.23 & - & - & - & 100.3 & & 72.1 & \\
\hline & & & & SD & & & 0.3 & 0.04 & 0.2 & & 0.9 & 0.02 & 0.5 & 0.6 & 0.08 & & & & 0.3 & & 2.4 & \\
\hline & & & Opx & $N=6$ & Anhedral & & 52.7 & 0.54 & 0.9 & - & 20.9 & 0.42 & 23.6 & 2.1 & - & - & - & - & 101.6 & & 66.8 & \\
\hline & & & & SD & & & 0.3 & 0.03 & 0.1 & & 0.2 & 0.03 & 0.3 & 0.2 & & & & & 0.4 & & 0.4 & \\
\hline \multirow[t]{14}{*}{$231 \mathrm{R}-3,59-63$} & 1491.15 & Gabbro 2 & $\mathrm{Pl}$ & $N=3$ & Subhedral & Core & 50.9 & 0.10 & 29.9 & - & 0.6 & - & 0.1 & 13.5 & 3.94 & - & - & - & 99.1 & 65.4 & & \\
\hline & & & & SD & & & 3.0 & 0.02 & 1.9 & & 0.0 & & 0.0 & 2.2 & 1.24 & & & & 0.2 & 10.9 & & \\
\hline & & & $\mathrm{Pl}$ & $N=1$ & Subhedral & Rim & 53.3 & 0.08 & 28.5 & - & 0.4 & - & 0.0 & 11.7 & 5.07 & - & - & - & 99.2 & 56.1 & & \\
\hline & & & & SD & & & & & & & & & & & & & & & & & & \\
\hline & & & Cpx & $N=6$ & Igneous-type & Core & 52.2 & 0.43 & 2.0 & 0.27 & 7.3 & 0.14 & 17.3 & 20.4 & 0.24 & - & - & - & 100.5 & & 80.8 & \\
\hline & & & & SD & & & 0.3 & 0.04 & 0.1 & 0.04 & 0.9 & 0.04 & 0.5 & 0.4 & 0.02 & & & & 0.4 & & 2.2 & \\
\hline & & & Cpx & $N=3$ & Amphibole-type & Core & 52.7 & 0.16 & 0.8 & - & 8.7 & 0.20 & 14.9 & 22.8 & 0.22 & - & - & - & 100.6 & & 75.5 & \\
\hline & & & & SD & & & 0.3 & 0.02 & 0.3 & & 0.3 & 0.05 & 0.2 & 0.4 & 0.01 & & & & 0.5 & & 0.8 & \\
\hline & & & Opx & $N=6$ & Anhedral & & 52.9 & 0.53 & 0.8 & - & 20.8 & 0.38 & 23.8 & 2.1 & - & - & - & - & 101.5 & & 67.1 & \\
\hline & & & & SD & & & 0.4 & 0.12 & 0.1 & & 0.7 & 0.04 & 0.3 & 0.4 & & & & & 0.7 & & 0.9 & \\
\hline & & & $\mathrm{Ol}$ & $N=2$ & Interstitial & Core & 38.0 & - & 0.0 & - & 29.4 & 0.44 & 34.3 & 0.0 & & & 0.07 & & 102.3 & & & 67.6 \\
\hline & & & & SD & & & 0.1 & - & - & - & 0.0 & 0.00 & 0.4 & 0.0 & & & 0.00 & & 0.4 & & & 0.2 \\
\hline & & & $\mathrm{Ol}$ & $N=2$ & Interstitial & $\operatorname{Rim}$ & 38.1 & - & 0.0 & - & 28.7 & 0.44 & 34.8 & 0.0 & & & 0.06 & & 102.1 & & & 68.4 \\
\hline & & & & SD & & & 0.0 & - & - & - & 0.1 & 0.05 & 0.4 & 0.0 & & & 0.00 & & 0.4 & & & 0.2 \\
\hline \multirow[t]{13}{*}{$231 \mathrm{R}-4,70-74$} & 1492.63 & Gabbro 2 & $\mathrm{PI}$ & $N=8$ & Subhedral & Core & 51.7 & 0.12 & 29.8 & - & 0.5 & - & 0.0 & 13.0 & 4.14 & - & - & - & 99.3 & 63.5 & & \\
\hline & & & & SD & & & 1.1 & & 0.8 & & 0.1 & & 0.0 & 0.9 & 0.49 & & & & 0.2 & 4.4 & & \\
\hline & & & $\mathrm{Pl}$ & $\begin{array}{l}N=1 \\
S D\end{array}$ & Subhedral & Rim & 52.7 & - & 28.8 & - & 0.8 & - & 0.0 & 12.1 & 4.66 & - & - & - & 99.2 & 59.0 & & \\
\hline & & & Cpx & $N=7$ & Igneous-type & Core & 51.5 & 0.64 & 1.9 & 0.23 & 8.7 & 0.18 & 15.5 & 21.0 & 0.24 & - & - & - & 100.0 & & 75.9 & \\
\hline & & & & SD & & & 0.4 & 0.16 & 0.4 & 0.07 & 1.6 & 0.05 & 1.4 & 0.7 & 0.02 & & & & 0.5 & & 4.7 & \\
\hline & & & $\mathrm{Cpx}$ & $N=6$ & Amphibole-type & Core & 52.8 & 0.09 & 0.5 & - & 7.9 & 0.14 & 15.1 & 22.8 & 0.31 & - & - & - & 99.8 & & 77.3 & \\
\hline & & & & SD & & & 0.1 & 0.01 & 0.2 & & 1.0 & 0.02 & 0.5 & 0.7 & 0.13 & & & & 0.3 & & 2.7 & \\
\hline & & & Opx & $N=9$ & Anhedral & & 52.6 & 0.48 & 1.0 & - & 19.9 & 0.36 & 23.9 & 1.9 & - & - & - & - & 100.4 & & 68.1 & \\
\hline & & & & SD & & & 0.1 & 0.06 & 0.1 & & 0.4 & 0.06 & 0.2 & 0.2 & & & & & 0.4 & & 0.6 & \\
\hline & & & $\mathrm{Ol}$ & $N=3$ & Anhedral & Core & 38.8 & - & - & - & 25.1 & 0.41 & 37.6 & 0.0 & & & 0.08 & & 102.0 & & & 72.7 \\
\hline & & & & SD & & & 0.2 & - & - & - & 0.7 & 0.01 & 0.5 & 0.0 & & & 0.01 & & 0.1 & & & 0.8 \\
\hline & & & $\mathrm{Ol}$ & $N=3$ & Anhedral & $\operatorname{Rim}$ & 39.1 & - & 0.0 & - & 24.5 & 0.38 & 38.4 & 0.0 & & & 0.08 & & 102.5 & & & 73.6 \\
\hline & & & & SD & & & 0.5 & - & 0.0 & - & 0.8 & 0.03 & 0.9 & 0.0 & & & 0.00 & & 0.7 & & & 1.1 \\
\hline \multirow[t]{7}{*}{$232 \mathrm{R}-1,36-39$} & 1493.26 & Gabbro 2 & $\mathrm{Pl}$ & $N=1$ & Subhedral & Core & 54.2 & - & 27.7 & - & 0.6 & - & 0.1 & 11.0 & 5.17 & - & - & - & 98.9 & 54.0 & & \\
\hline & & & & SD & & & & & & & & & & & & & & & & & & \\
\hline & & & $\mathrm{PI}$ & $\begin{array}{l}N=1 \\
S D\end{array}$ & Subhedral & $\operatorname{Rim}$ & 53.4 & - & 28.4 & - & 0.4 & - & 0.0 & 11.6 & 5.13 & - & - & - & 99.0 & 55.6 & & \\
\hline & & & Cpx & $N=3$ & Igneous-type & Core & 51.7 & 0.55 & 1.7 & - & 10.6 & 0.29 & 14.9 & 20.4 & 0.39 & - & - & - & 100.8 & & 71.4 & \\
\hline & & & & SD & & & 0.6 & 0.16 & 0.4 & & 0.9 & 0.03 & 0.4 & 0.7 & 0.17 & & & & 0.4 & & 1.2 & \\
\hline & & & Cpx & $N=6$ & Amphibole-type & Core & 52.4 & 0.26 & 1.8 & - & 8.3 & 0.23 & 15.1 & 21.8 & 0.50 & - & - & - & 100.5 & & 76.4 & \\
\hline & & & & SD & & & 0.3 & 0.07 & 1.1 & & 0.5 & 0.03 & 0.6 & 1.0 & 0.29 & & & & 0.6 & & 1.5 & \\
\hline
\end{tabular}


Table T3 (continued). (Continued on next page.)

\begin{tabular}{|c|c|c|c|c|c|c|c|c|c|c|c|c|c|c|c|c|c|c|c|c|c|c|}
\hline \multirow{2}{*}{$\begin{array}{l}\text { Core, section, } \\
\text { interval }(\mathrm{cm})\end{array}$} & \multirow{2}{*}{$\begin{array}{l}\text { Depth } \\
\text { (mbsf) }\end{array}$} & \multirow[b]{2}{*}{ Rock name } & \multirow[b]{2}{*}{ Phase } & \multirow[b]{2}{*}{ Replicates } & \multirow{2}{*}{$\begin{array}{l}\text { Mode of } \\
\text { occurrence }\end{array}$} & \multirow[b]{2}{*}{ AP } & \multicolumn{16}{|c|}{ Major element oxide (wt\%) } \\
\hline & & & & & & & $\mathrm{SiO}_{2}$ & $\mathrm{TiO}_{2}$ & $\mathrm{Al}_{2} \mathrm{O}_{3}$ & $\mathrm{Cr}_{2} \mathrm{O}_{3}$ & $\mathrm{FeO}$ & $\mathrm{MnO}$ & $\mathrm{MgO}$ & $\mathrm{CaO}$ & $\mathrm{Na}_{2} \mathrm{O}$ & $\mathrm{K}_{2} \mathrm{O}$ & $\mathrm{NiO}$ & $\mathrm{BaO}$ & Total & $\mathrm{An} \%$ & Mg\# & Fo \\
\hline & & & Opx & $N=6$ & Anhedral & & 52.9 & 0.42 & 0.8 & - & 20.1 & 0.42 & 24.5 & 1.9 & - & - & - & - & 101.1 & & 68.5 & \\
\hline & & & & SD & & & 0.5 & 0.17 & 0.2 & & 0.5 & 0.03 & 0.5 & 0.4 & & & & & 0.6 & & 1.0 & \\
\hline & & & Ol & $N=11$ & Anhedral & Core & 38.4 & - & 0.0 & - & 27.5 & 0.40 & 36.0 & 0.0 & & & 0.06 & & 102.4 & & & 70.0 \\
\hline & & & & SD & & & 0.2 & - & 0.0 & - & 0.4 & 0.01 & 0.4 & 0.0 & & & 0.01 & & 0.3 & & & 0.6 \\
\hline & & & $\mathrm{Ol}$ & $N=7$ & Anhedral & $\operatorname{Rim}$ & 38.5 & - & 0.0 & - & 27.1 & 0.40 & 36.4 & 0.1 & & & 0.07 & & 102.6 & & & 70.6 \\
\hline & & & & SD & & & 0.2 & - & 0.0 & - & 0.4 & 0.01 & 0.4 & 0.0 & & & 0.01 & & 0.2 & & & 0.5 \\
\hline \multirow[t]{12}{*}{$232 \mathrm{R}-1,78-82$} & 1493.68 & Gabbro 2 & $\mathrm{Pl}$ & $N=6$ & Subhedral & Core & 51.1 & - & 30.1 & - & 0.6 & - & 0.0 & 13.6 & 3.82 & - & - & - & 99.3 & 66.3 & & \\
\hline & & & & SD & & & 0.7 & & 0.6 & & 0.0 & & 0.0 & 0.7 & 0.52 & & & & 0.1 & 4.2 & & \\
\hline & & & $\mathrm{PI}$ & $N=5$ & Subhedral & $\operatorname{Rim}$ & 55.4 & - & 27.6 & - & 0.4 & - & 0.0 & 10.1 & 5.96 & - & - & - & 99.5 & 48.4 & & \\
\hline & & & & SD & & & 1.1 & & 0.8 & & 0.1 & & 0.0 & 1.0 & 0.50 & & & & 0.3 & 4.5 & & \\
\hline & & & $\mathrm{Cpx}$ & $N=5$ & Igneous-type & Core & 51.4 & 0.62 & 1.8 & 0.18 & 9.3 & 0.24 & 15.6 & 20.6 & 0.30 & - & - & - & 100.1 & & 74.9 & \\
\hline & & & & SD & & & 0.3 & 0.10 & 0.2 & & 0.5 & 0.07 & 0.1 & 0.6 & 0.02 & & & & 0.4 & & 0.8 & \\
\hline & & & Cpx & $N=14$ & Amphibole-type & Core & 52.5 & 0.10 & 0.6 & - & 8.5 & 0.23 & 14.7 & 23.0 & 0.21 & - & - & - & 100.1 & & 75.5 & \\
\hline & & & & SD & & & 0.4 & 0.04 & 0.2 & & 0.6 & 0.06 & 0.3 & 0.3 & 0.06 & & & & 0.4 & & 1.6 & \\
\hline & & & Opx & $N=14$ & Anhedral & & 52.8 & 0.46 & 0.9 & - & 18.9 & 0.40 & 24.8 & 2.0 & - & - & - & - & 100.3 & & 70.1 & \\
\hline & & & & SD & & & 0.2 & 0.04 & 0.2 & & 0.8 & 0.03 & 0.4 & 0.1 & & & & & 0.4 & & 1.2 & \\
\hline & & & Opx & $N=3$ & Granular & & 54.2 & 0.27 & 0.8 & - & 17.1 & 0.38 & 27.3 & 0.9 & - & - & - & - & 101.0 & & 72.6 & \\
\hline & & & & SD & & & 0.9 & 0.08 & 0.3 & & 1.2 & 0.03 & 1.7 & 0.8 & & & & & 0.4 & & 0.4 & \\
\hline \multirow[t]{6}{*}{$232 \mathrm{R}-2,10-14$} & 1494.08 & Gabbro 2 & $\mathrm{Pl}$ & $\begin{array}{l}N=1 \\
S D\end{array}$ & Subhedral & Core & 52.2 & - & 29.2 & - & 0.6 & - & 0.1 & 12.3 & 4.43 & - & - & - & 98.8 & 60.5 & & \\
\hline & & & $\mathrm{Pl}$ & $\begin{array}{l}N=1 \\
S D\end{array}$ & Subhedral & Rim & 54.4 & - & 28.1 & - & 0.5 & - & 0.1 & 10.6 & 5.42 & - & - & - & 99.1 & 51.9 & & \\
\hline & & & $C p x$ & $N=7$ & Amphibole-type & Core & 53.2 & 0.14 & 0.7 & - & 9.0 & 0.23 & 15.0 & 22.4 & 0.33 & - & - & - & 101.1 & & 74.9 & \\
\hline & & & & SD & & & 0.4 & 0.10 & 0.4 & & 0.6 & 0.05 & 0.6 & 0.9 & 0.09 & & & & 0.3 & & 1.7 & \\
\hline & & & Opx & $N=8$ & Anhedral & & 53.1 & 0.48 & 0.7 & - & 20.7 & 0.45 & 24.5 & 1.8 & - & - & - & - & 102.0 & & 67.9 & \\
\hline & & & & SD & & & 0.2 & 0.02 & 0.1 & & 0.9 & 0.05 & 0.5 & 0.2 & & & & & 0.4 & & 1.4 & \\
\hline \multirow[t]{10}{*}{ 232R-2, 73-76 } & 1494.71 & Gabbro 2 & $\mathrm{PI}$ & $N=4$ & Subhedral & Core & 49.4 & - & 31.0 & - & 0.7 & - & 0.1 & 15.1 & 3.04 & - & - & - & 99.4 & 73.3 & & \\
\hline & & & & SD & & & 1.0 & & 0.6 & & 0.1 & & 0.0 & 0.6 & 0.40 & & & & 0.2 & 3.4 & & \\
\hline & & & $\mathrm{PI}$ & $N=3$ & Subhedral & $\operatorname{Rim}$ & 50.8 & - & 30.1 & - & 0.7 & - & 0.1 & 13.7 & 3.70 & - & - & - & 99.1 & 67.2 & & \\
\hline & & & & SD & & & 1.7 & & 0.9 & & 0.2 & & 0.0 & 1.5 & 0.83 & & & & 0.1 & 7.3 & & \\
\hline & & & $C p x$ & $N=2$ & Igneous-type & Core & 52.9 & 0.30 & 1.9 & 0.56 & 5.5 & 0.18 & 18.3 & 20.8 & 0.20 & - & - & - & 100.7 & & 85.5 & \\
\hline & & & & SD & & & 0.0 & 0.01 & 0.0 & 0.07 & 0.0 & 0.01 & 0.0 & 0.0 & 0.00 & & & & 0.1 & & 0.0 & \\
\hline & & & $\mathrm{Cpx}$ & $N=3$ & Amphibole-type & Core & 52.1 & 0.44 & 1.4 & 0.18 & 9.9 & 0.25 & 15.1 & 20.6 & 0.25 & - & - & - & 100.3 & & 73.2 & \\
\hline & & & & SD & & & 0.7 & 0.17 & 0.6 & & 1.4 & 0.06 & 0.5 & 1.3 & 0.01 & & & & 0.6 & & 3.3 & \\
\hline & & & Opx & $N=8$ & Anhedral & & 53.3 & 0.48 & 0.9 & - & 18.7 & 0.40 & 25.4 & 1.9 & - & - & - & - & 101.1 & & 70.8 & \\
\hline & & & & SD & & & 0.3 & 0.06 & 0.2 & & 0.9 & 0.06 & 0.8 & 0.2 & & & & & 0.6 & & 1.6 & \\
\hline \multirow[t]{11}{*}{$233 \mathrm{R}-1,4-7$} & 1497.50 & Type 8 & $\mathrm{PI}$ & $N=3$ & Subhedral & Core & 53.4 & - & 28.7 & - & 0.5 & - & 0.0 & 11.6 & 4.96 & - & - & - & 99.3 & 56.4 & & \\
\hline & & & & SD & & & 1.3 & & 0.8 & & 0.0 & & 0.0 & 0.9 & 0.45 & & & & 0.4 & 4.0 & & \\
\hline & & & $\mathrm{PI}$ & $N=2$ & Subhedral & Rim & 54.1 & - & 28.2 & - & 0.6 & - & 0.0 & 11.1 & 5.34 & - & - & - & 99.5 & 53.4 & & \\
\hline & & & & SD & & & 0.5 & & 0.0 & & 0.0 & & 0.0 & 0.0 & 0.06 & & & & 0.6 & 0.1 & & \\
\hline & & & Cpx & $N=2$ & Igneous-type & Core & 51.3 & 0.62 & 1.7 & - & 10.4 & 0.25 & 15.1 & 20.4 & 0.35 & - & - & - & 100.2 & & 72.1 & \\
\hline & & & & SD & & & 0.5 & 0.09 & 0.4 & & 0.5 & 0.04 & 0.1 & 0.7 & 0.06 & & & & 0.7 & & 0.9 & \\
\hline & & & $C p x$ & $N=3$ & Amphibole-type & Core & 53.5 & 0.11 & 0.5 & - & 7.2 & 0.18 & 15.7 & 22.9 & 0.32 & - & - & - & 100.6 & & 79.7 & \\
\hline & & & & SD & & & 0.1 & 0.02 & 0.1 & & 0.1 & 0.07 & 0.1 & 0.3 & 0.04 & & & & 0.3 & & 0.3 & \\
\hline & & & $C p x$ & $N=3$ & Secondary-type & Core & 53.2 & 0.08 & 0.3 & - & 8.6 & 0.16 & 14.8 & 22.7 & 0.55 & - & - & - & 100.5 & & 75.5 & \\
\hline & & & & SD & & & 0.1 & 0.02 & 0.1 & & 1.0 & 0.03 & 0.7 & 0.5 & 0.22 & & & & 0.4 & & 2.9 & \\
\hline & & & Opx & $N=15$ & Microgranular & & 52.9 & 0.39 & 0.8 & - & 20.5 & 0.41 & 23.8 & 1.9 & - & - & - & - & 100.9 & & 67.4 & \\
\hline
\end{tabular}


Table T3 (continued).

\begin{tabular}{|c|c|c|c|c|c|c|c|c|c|c|c|c|c|c|c|c|c|c|c|c|c|c|}
\hline \multirow{2}{*}{$\begin{array}{l}\text { Core, section, } \\
\text { interval }(\mathrm{cm})\end{array}$} & \multirow{2}{*}{$\begin{array}{l}\text { Depth } \\
\text { (mbsf) }\end{array}$} & \multirow[b]{2}{*}{ Rock name } & \multirow[b]{2}{*}{ Phase } & \multirow[b]{2}{*}{ Replicates } & \multirow{2}{*}{$\begin{array}{l}\text { Mode of } \\
\text { occurrence }\end{array}$} & \multirow[b]{2}{*}{ AP } & \multicolumn{16}{|c|}{ Major element oxide (wt\%) } \\
\hline & & & & & & & $\mathrm{SiO}_{2}$ & $\mathrm{TiO}_{2}$ & $\mathrm{Al}_{2} \mathrm{O}_{3}$ & $\mathrm{Cr}_{2} \mathrm{O}_{3}$ & $\mathrm{FeO}$ & $\mathrm{MnO}$ & $\mathrm{MgO}$ & $\mathrm{CaO}$ & $\mathrm{Na}_{2} \mathrm{O}$ & $\mathrm{K}_{2} \mathrm{O}$ & $\mathrm{NiO}$ & $\mathrm{BaO}$ & Total & An\% & Mg\# & Fo \\
\hline & & & & SD & & & 0.2 & 0.04 & 0.1 & & 0.4 & 0.04 & 0.2 & 0.2 & & & & & 0.6 & & 0.5 & \\
\hline \multirow[t]{11}{*}{$234 \mathrm{R}-1,1-2$} & 1502.50 & $\begin{array}{l}\text { Type } 8 \\
\text { metabasalt; } \\
\text { LDS }\end{array}$ & $\mathrm{PI}$ & $N=2$ & Phenocryst & Core & 49.8 & - & 30.8 & - & 0.8 & - & 0.0 & 14.7 & 3.17 & - & - & - & 99.3 & 72.0 & & \\
\hline & & & & SD & & & 0.3 & & 0.1 & & 0.1 & & 0.0 & 0.1 & 0.14 & & & & 0.1 & 0.8 & & \\
\hline & & & $\mathrm{PI}$ & $N=2$ & Phenocryst & $\operatorname{Rim}$ & 54.5 & 0.09 & 28.1 & - & 0.7 & - & 0.0 & 11.2 & 5.24 & - & - & - & 99.8 & 54.1 & & \\
\hline & & & & SD & & & 0.4 & - & 0.1 & & 0.2 & & 0.0 & 0.0 & 0.23 & & & & 0.4 & 1.2 & & \\
\hline & & & $\mathrm{PI}$ & $N=5$ & Microgranular & Core & 54.3 & - & 28.1 & - & 0.6 & - & 0.0 & 11.0 & 5.46 & - & - & - & 99.5 & 52.7 & & \\
\hline & & & & SD & & & 0.3 & & 0.2 & & 0.0 & & 0.0 & 0.2 & 0.08 & & & & 0.3 & 0.7 & & \\
\hline & & & Cpx & $N=4$ & Phenocryst & Core & 51.4 & 0.54 & 1.6 & 0.33 & 9.9 & 0.22 & 14.9 & 21.1 & 0.30 & - & - & - & 100.2 & & 72.8 & \\
\hline & & & & SD & & & 0.3 & 0.14 & 0.3 & 0.17 & 0.5 & 0.02 & 0.1 & 0.4 & 0.06 & & & & 0.4 & & 1.1 & \\
\hline & & & Cpx & $\begin{array}{l}N=1 \\
S D\end{array}$ & Phenocryst & Rim & 51.4 & 0.63 & 1.8 & - & 9.6 & 0.24 & 14.6 & 21.5 & 0.32 & - & - & - & 100.3 & & 73.1 & \\
\hline & & & Cpx & $N=6$ & Microgranular & Core & 51.7 & 0.54 & 1.3 & - & 9.9 & 0.26 & 15.0 & 21.2 & 0.24 & - & - & - & 100.2 & & 73.1 & \\
\hline & & & & SD & & & 0.3 & 0.12 & 0.2 & & 0.6 & 0.05 & 0.2 & 0.7 & 0.03 & & & & 0.4 & & 0.9 & \\
\hline \multirow[t]{12}{*}{$234 \mathrm{R}-1,7-9$} & 1502.57 & $\begin{array}{l}\text { Type } 8 \\
\text { metabasalt; } \\
\text { LDS }\end{array}$ & $\mathrm{PI}$ & $N=3$ & Phenocryst & Core & 50.7 & - & 30.4 & - & 0.6 & - & - & 14.0 & 3.47 & - & - & - & 99.3 & 69.1 & & \\
\hline & & & & SD & & & 0.2 & & 0.2 & & 0.1 & & & 0.2 & 0.06 & & & & 0.3 & 0.6 & & \\
\hline & & & $\mathrm{PI}$ & $N=2$ & Phenocryst & $\operatorname{Rim}$ & 52.6 & - & 29.3 & - & 0.5 & - & 0.0 & 12.5 & 4.42 & - & - & - & 99.3 & 61.0 & & \\
\hline & & & & SD & & & 2.9 & & 1.6 & & 0.0 & & 0.0 & 2.2 & 1.31 & & & & 0.4 & 11.2 & & \\
\hline & & & $\mathrm{Pl}$ & $N=2$ & Microgranular & Core & 54.3 & - & 28.1 & - & 0.6 & - & 0.0 & 11.2 & 5.17 & - & - & - & 99.4 & 54.6 & & \\
\hline & & & & SD & & & 0.6 & & 0.2 & & 0.0 & & 0.0 & 0.2 & 0.34 & & & & 0.5 & 2.1 & & \\
\hline & & & Cpx & $\begin{array}{l}N=1 \\
S D\end{array}$ & Phenocryst & Core & 50.8 & 0.58 & 1.5 & - & 9.2 & 0.25 & 14.5 & 22.0 & 0.32 & - & - & - & 99.2 & & 73.8 & \\
\hline & & & Cpx & $\begin{array}{l}N=1 \\
S D\end{array}$ & Phenocryst & Rim & 51.4 & 0.54 & 1.3 & - & 9.6 & 0.23 & 14.9 & 21.3 & 0.30 & - & - & - & 99.6 & & 73.4 & \\
\hline & & & Cpx & $N=6$ & Microgranular & Core & 51.8 & 0.56 & 1.3 & - & 10.0 & 0.25 & 15.0 & 20.9 & 0.27 & - & - & - & 100.0 & & 72.7 & \\
\hline & & & & SD & & & 0.3 & 0.13 & 0.1 & & 0.5 & 0.05 & 0.2 & 0.5 & 0.03 & & & & 0.5 & & 0.9 & \\
\hline & & & Opx & $N=12$ & Microgranular & & 52.5 & 0.41 & 0.8 & - & 21.4 & 0.43 & 23.1 & 1.7 & - & - & - & - & 100.5 & & 65.8 & \\
\hline & & & & SD & & & 0.3 & 0.04 & 0.1 & & 0.4 & 0.04 & 0.3 & 0.2 & & & & & 0.6 & & 0.6 & \\
\hline \multicolumn{23}{|l|}{ Detection limits: } \\
\hline $\mathrm{Pl}, \mathrm{Cpx}$, and Opx & & & & & & & 0.0 & 0.07 & 0.0 & 0.14 & 0.1 & 0.10 & 0.0 & 0.0 & 0.06 & 0.07 & 0.12 & 0.16 & & & & \\
\hline Ol & & & & & & & 0.0 & 0.04 & 0.0 & 0.04 & 0.0 & 0.02 & 0.0 & 0.0 & & & 0.02 & & & & & \\
\hline
\end{tabular}

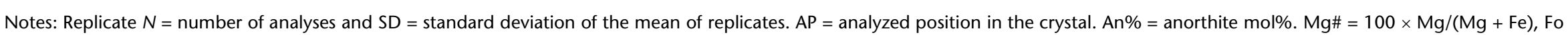
$=$ forsterite $\mathrm{mol} \%$. UDS = upper dike screen, $\mathrm{LDS}=$ lower dike screen. $\mathrm{PI}=$ plagioclase, $\mathrm{Cpx}=$ clinopyroxene, Opx = orthopyroxene, $\mathrm{OI}=$ olivine. $-=$ below detection limit, blank cell $=$ not analyzed. 